\title{
Characterization and Application of Novel Structure Switching Aptamers for Aflatoxins
}

\author{
by
}

Daniel Goudreau

A thesis submitted to the Faculty of Graduate and Postdoctoral Affairs in partial fulfilment of the requirements for the degree of

Master of Science

in

Chemistry

Carleton University

Ottawa, Ontario

C 2017, Daniel Goudreau 


\begin{abstract}
Aflatoxins are a class of mycotoxins that are of great agro-economic importance. Their severe acute and chronic health effects have made them of great concern from a public health perspective as well. Due to their health implications, and large economic burden, aflatoxins have become the most studied group of mycotoxins. A specific interest in rapid, robust, and cost effective, on-site sensing platforms for mycotoxin contaminants has been shown. Aptamers are functional oligonucleotide sequences that are selected to bind a cognate ligand with high affinity and selectivity. Novel structure switching aptamer candidates for total aflatoxin were previously selected. The selection was performed using two template designs. Electrophoretic mobility shift assays, melting temperature studies, and circular dichroism along with predictive structural characterization techniques were used to help elucidate the aptamer candidate's structural biases for G-quadruplex formation. Two candidates were determined to bind aflatoxin $\mathrm{B}_{1}$ $\left(\mathrm{AFB}_{1}\right)$ with high affinity using microscale thermophoresis. Sequences DZA3 and 3MR5 had reported dissociation constants $\left(\mathrm{K}_{\mathrm{d}}\right)$ for $\mathrm{AFB}_{1}$ of $42.1 \pm 23.8 \mathrm{nM}$ and $1.4 \pm 6.7 \mu \mathrm{M}$, respectively. Using the $\mathrm{AFB}_{1}$ binding aptamers, progress has been made towards developing FRET-based signalling sensors that leverage the structure-switching properties of the aptamer.
\end{abstract}




\section{Acknowledgements}

First and foremost, I would like to thank Dr. Maria DeRosa for allowing me the opportunity to work in the lab. You have given me so many opportunities to learn and grow, and I truly appreciate that! With so many people, it is hard to list everyone, but I am glad I had the chance to meet every one of you. You have all made this an unforgettable experience. I wish you all the best of luck moving forward.

I want to thank a few people I have gotten to know well. Emily, you are hilarious and your dark sense of humour is refreshing. That's probably not something you ever expect to hear, but it's mostly because I don't have to feel like I'm the only one that thinks that way. McKenzie, thank you for paving the way. I enjoyed getting to experience this with you. Matthew, I have known you since I first started at Carleton. I want to say that you have unwavering optimism. You always provide great advice, and even better food! Christopher, I am glad I had the chance to work with someone that shares a lot of the same interests as me; you know like cars, dogs, and throwing sharp objects. Good luck with the remainder of your $\mathrm{PhD}$. Thank you, Brandon, for all the last-minute help. Thank you, Erin, for being a voice of reason. I imagine you will do great at McMaster. Also, I will never forget the time you called me out for falling asleep in your lecture...

To my great friend Simon, I know you don't understand a lot of what I do but thank you for still humouring me. I'm grateful for the time I get to spend outdoors, and I can't wait to get back in the woods. I would also like to thank my parents for giving me so many opportunities in life and for supporting me in this endeavour. I want to thank you Kaylyn for your never-ending love and support. I am fortunate to have such a strong partner. Also, thank you for doing all the dishes while I have been writing my thesis. 
Finally, I want to thank my "therapy" dog Frankie. She can't read this so I'll be honest. She drives me up the wall most of the time. But she is just so damn cute, I can't stay mad at her. 


\section{Table of Contents}

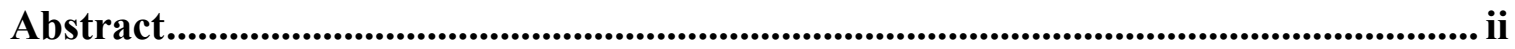

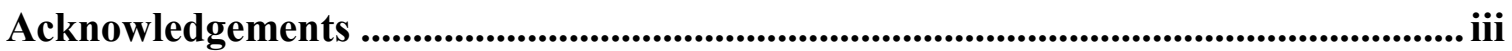

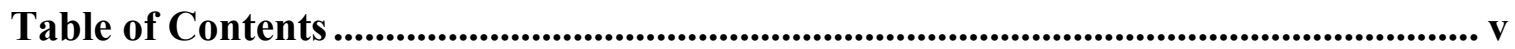

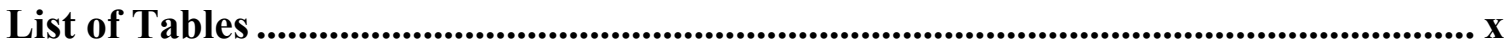

List of Figures.................................................................................................................................... xii

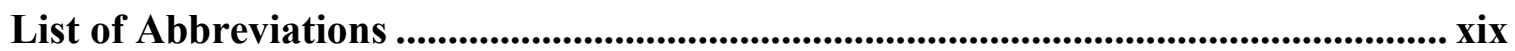

1 Chapter: Introduction ..................................................................................... 1

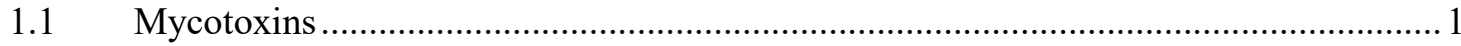

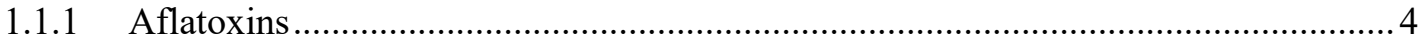

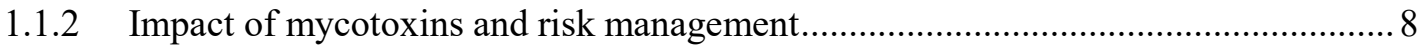

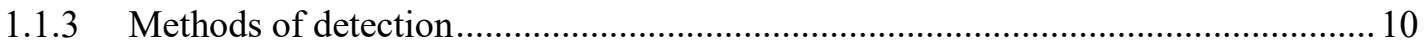

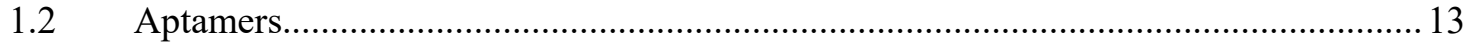

1.2.1 Systematic Evolution of Ligands by Exponential Enrichment (SELEX) ................ 16

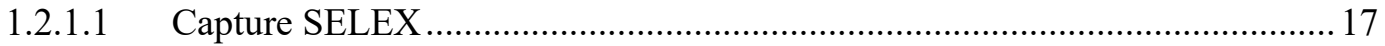

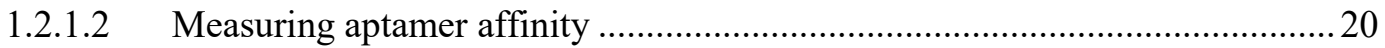

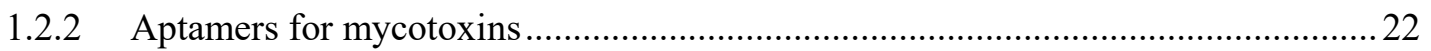

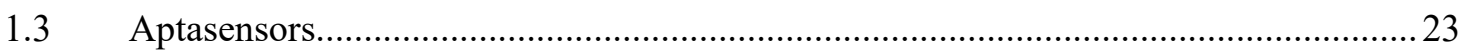

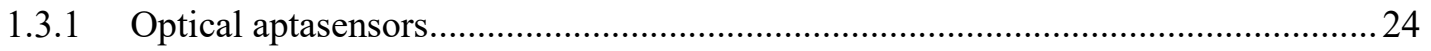

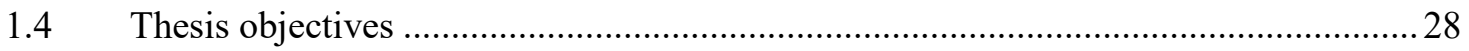

2 Chapter: Aptamer Characterization............................................................................ 29

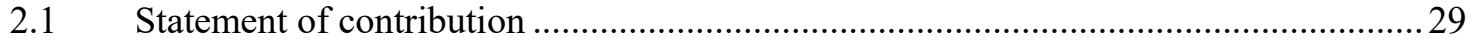

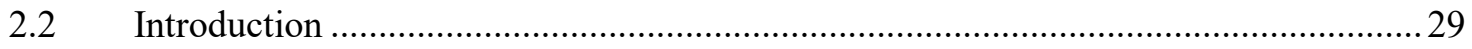

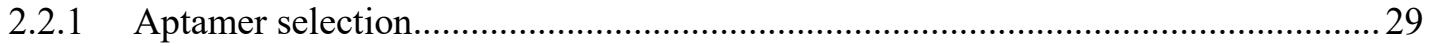

3.1.1 Structural characterization of nucleic acid aptamers............................................. 32 


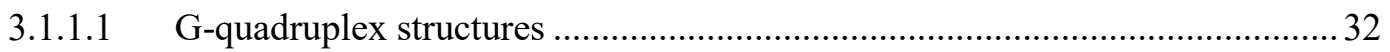

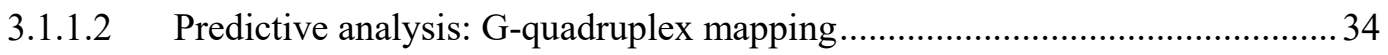

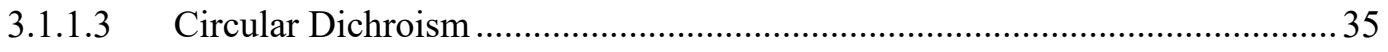

3.1.2 Measuring dissociation constants by microscale thermophoresis ........................... 37

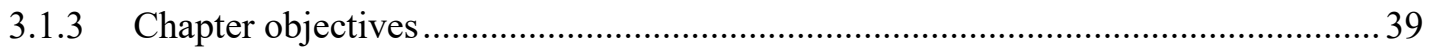

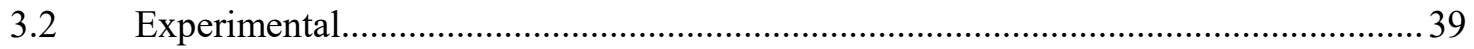

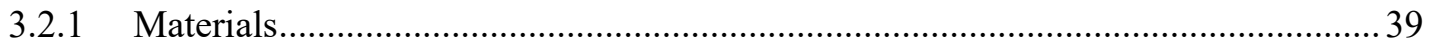

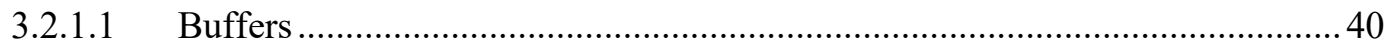

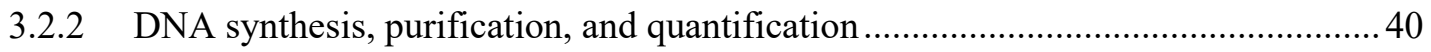

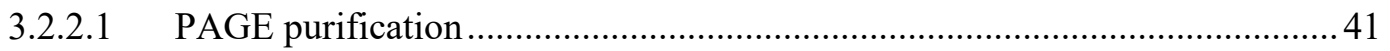

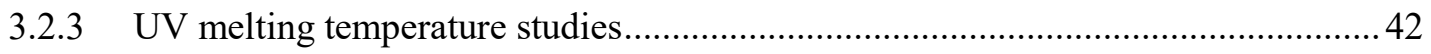

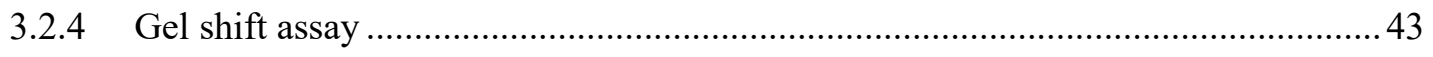

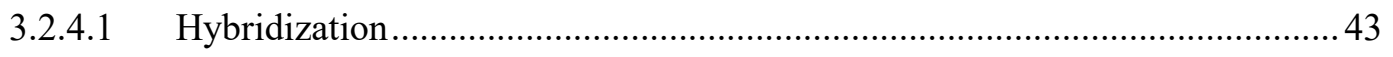

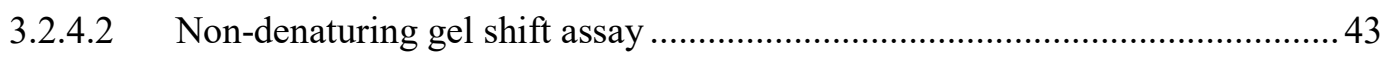

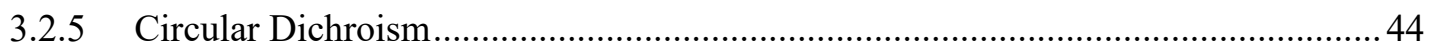

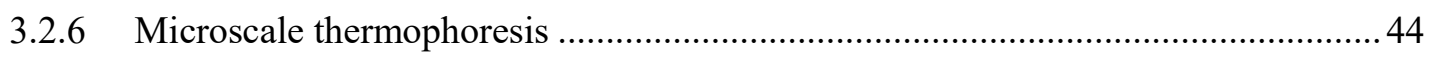

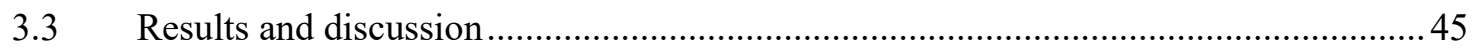

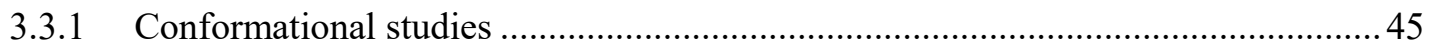

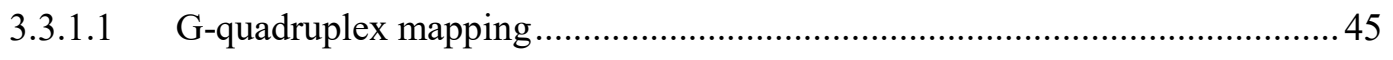

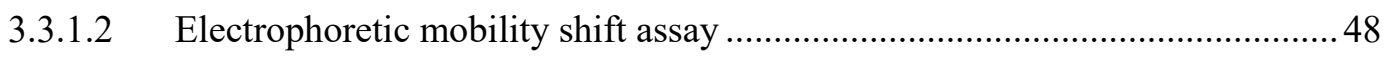

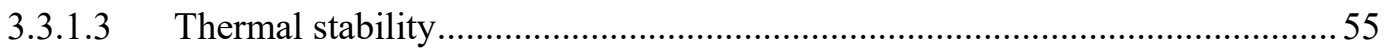

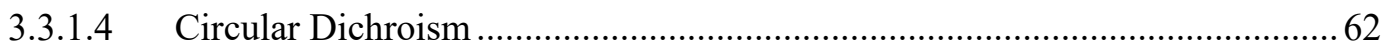

3.3.2 Dissociation constant $\left(\mathrm{K}_{\mathrm{d}}\right)$ determination using microscale thermophoresis ............66

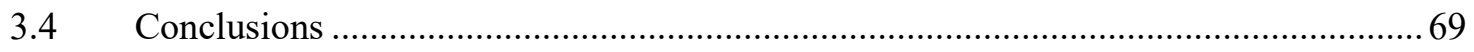

4 Chapter: Nanoaptasensors for Aflatoxin................................................................. 70

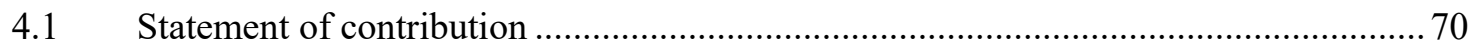




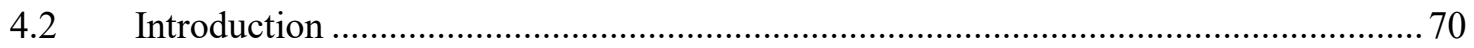

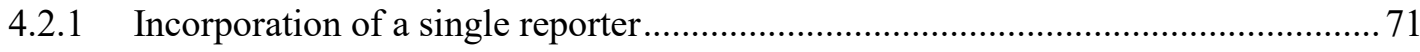

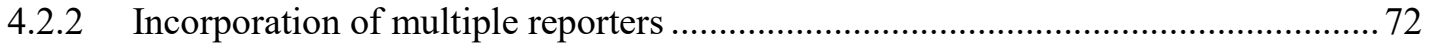

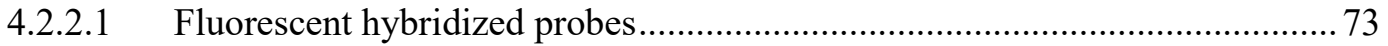

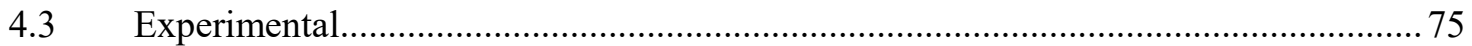

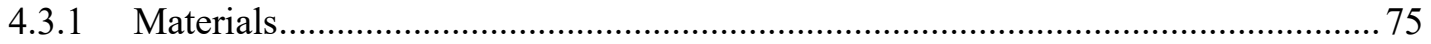

4.3.2 DNA synthesis, purification and quantification .................................................. 76

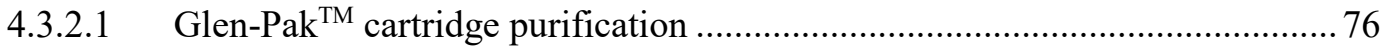

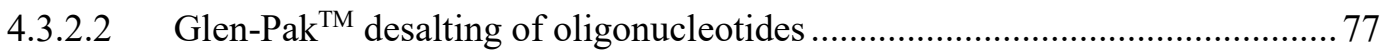

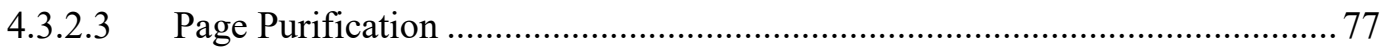

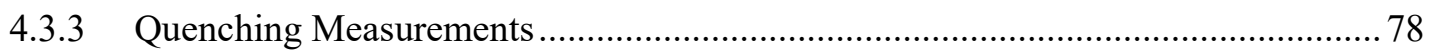

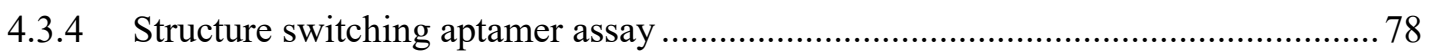

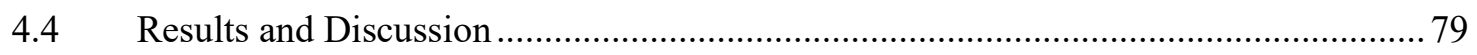

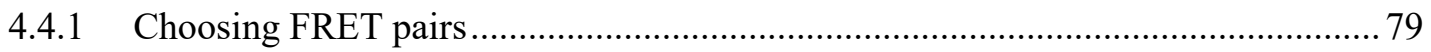

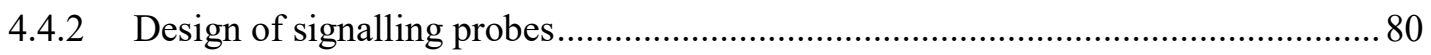

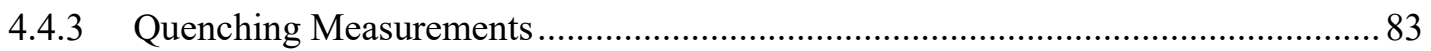

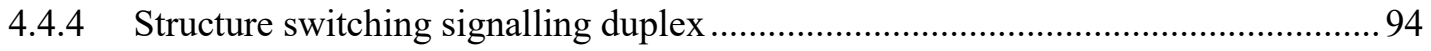

4.4.4.1 Sequence effect on fluorescein ............................................................... 98

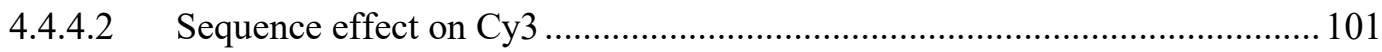

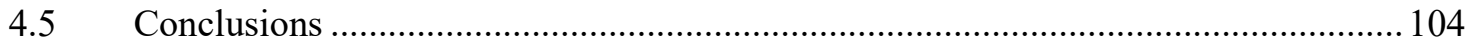

5 Chapter: Contributions to knowledge and future studies .................................. 105

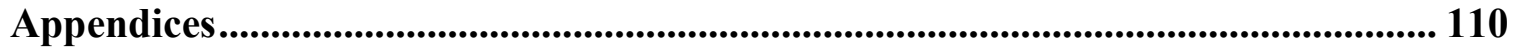

Appendix A : Melting Temperature Spectral Data.......................................................... 110

A.1 Melting temperature curve of hybridized DZA 3-FAM sequence and truncated

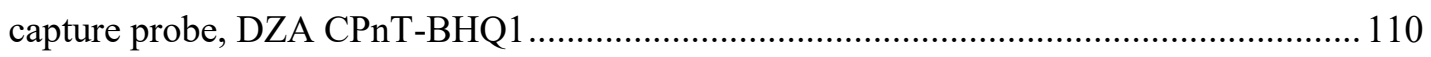

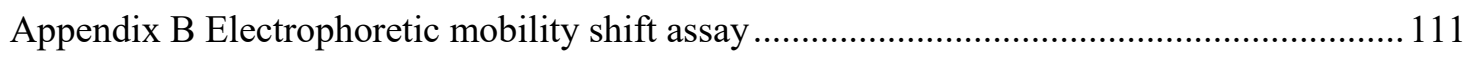




\section{B.1 Fluorescent images of structure switching DZA aptamer candidates by}

electrophoretic mobility shift assay when challenged with total aflatoxin mixture.

\section{B.2 Epi-UV images of structure switching DZA aptamer candidates by electrophoretic}

mobility shift assay when challenged with total aflatoxin mixture

B.3 Fluorescent images of structure switching 3MR (random) aptamer candidates by

electrophoretic mobility shift assay when challenged with total aflatoxin mixture.

B.4 Epi-UV images of structure switching 3MR (random) aptamer candidates by

electrophoretic mobility shift assay when challenged with total aflatoxin mixture.

Appendix C Microscale thermophoresis data.

C.1 Microscale thermophoresis results for DZA3 challenged with A) $\mathrm{AFB}_{2}$ with no measurable binding constant between $1.5 \mathrm{nM}$ and $50 \mu \mathrm{M}$ B) $\mathrm{AFG}_{1}$ with no measurable binding constant between $1.5 \mathrm{nM}$ and $50 \mu \mathrm{M}$.

C.2 Microscale thermophoresis results for A) DZA5 challenged with $\mathrm{AFB}_{1}$ showing no measurable binding constant between $1.5 \mathrm{nM}$ and $50 \mu \mathrm{M}$ B) DZA5 challenged with $\mathrm{AFB}_{2}$ with no measurable binding constant between $1.5 \mathrm{nM}$ and $50 \mu \mathrm{M}$. C) DZA5 challenged with $\mathrm{AFG}_{1}$ with no measurable binding constant between $1.5 \mathrm{nM}$ and $50 \mu \mathrm{M}$. D) DZA5 challenged with $\mathrm{AFG}_{2}$ with no measurable binding constant between $1.5 \mathrm{nM}$ and $50 \mu \mathrm{M} .114$ C.3 Microscale thermophoresis results for A) DZA7 challenged with $\mathrm{AFB}_{1}$ showing no measurable binding constant between $1.5 \mathrm{nM}$ and $50 \mu \mathrm{M}$ B) DZA5 challenged with $\mathrm{AFG}_{1}$ with no measurable binding constant between $1.5 \mathrm{nM}$ and $50 \mu \mathrm{M}$. C) DZA5 challenged with $\mathrm{AFG}_{2}$ with no measurable binding constant between $1.5 \mathrm{nM}$ and $50 \mu \mathrm{M}$.

\section{C.4 Microscale thermophoresis results for A) $3 \mathrm{MR} 2$ challenged with $\mathrm{AFB}_{1}$ showing no} measurable binding constant between $1.5 \mathrm{nM}$ and $50 \mu \mathrm{M}$ B) $3 \mathrm{MR} 2$ challenged with $\mathrm{AFB}_{2}$ with no measurable binding constant between $1.5 \mathrm{nM}$ and $50 \mu \mathrm{M} \mathrm{C}$ ) $3 \mathrm{MR} 2$ challenged with 
$\mathrm{AFG}_{1}$ with no measurable binding constant between $1.5 \mathrm{nM}$ and $50 \mu \mathrm{M}$. D) $3 \mathrm{MR} 2$ challenged with $\mathrm{AFG}_{2}$ with no measurable binding constant between $1.5 \mathrm{nM}$ and $50 \mu \mathrm{M} .116$ C.5 Microscale thermophoresis results for A) $3 \mathrm{MR} 5$ challenged with $\mathrm{AFB}_{2}$ showing no measurable binding constant between $1.526 \mathrm{nM}$ and $50 \mu \mathrm{M}$ B) $3 \mathrm{MR} 5$ challenged with $\mathrm{AFG}_{1}$ with no measurable binding constant between $1.526 \mathrm{nM}$ and $50 \mu \mathrm{M} \mathrm{C}$ ) 3MR5 challenged with $\mathrm{AFG}_{2}$ with no measurable binding constant between $1.526 \mathrm{nM}$ and $50 \mu \mathrm{M}$. 117

Appendix D CD Spectral Data 118

D.1 CD spectra of $3 \mathrm{M} \mathrm{Mg}$ buffer demonstrating highly positive bands below $220 \mathrm{~nm}$. 118

Appendix E Spectral data for quenched aptamer assemblies challenged with AF. 118

E.1 Fluorescence signal decrease with the addition of $1 \mu \mathrm{M} \mathrm{AFB}$ to the quenched 3MR5_FAM signalling aptamer at varying Apt/CP ratios A) 1:1, B) 1:2, C) 1:3, D) 1:4, E) 1:5, F) 3MR5_FAM and $\mathrm{AFB}_{1}$ only. The addition of aflatoxin results in further decreases in signal until maximal quenching is attained at higher ratios.

E.2 Fluorescence signal decrease with the addition of $1 \mu \mathrm{M}$ AFB1 to the quenched DZA3_Cy3 signalling aptamer at varying Apt/CP ratios A) 1:1, B) 1:2, C) 1:3, D) 1:4, E) 1:5, F) DZA3_Cy3 and AFB1 only. The addition of aflatoxin results in further decreases in signal until maximal quenching is attained at higher ratios.

E.3 Fluorescence signal decrease with the addition of $1 \mu \mathrm{M}$ AFB1 to the quenched 3MR5_Cy3 signalling aptamer at varying Apt/CP ratios A) 1:1, B) 1:2, C) 1:3, D) 1:4, E) 1:5, F) 3MR5_Cy3 and AFB1 only. The addition of aflatoxin results in further decreases in signal until maximal quenching is attained at higher ratios. 


\section{List of Tables}

Table 1.1: Important mycotoxin classes, their fungal producers, and commonly

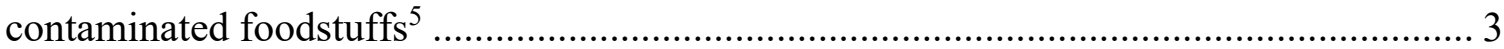

Table 1.2: Maximum tolerable levels for total aflatoxin contaminants in human food ${ }^{23} \ldots 8$

Table 1.3: Aptamers that have been previously selected for aflatoxin targets ................ 23

Table 1.4: Summary of recent aptasensors developed for aflatoxin contaminants (20142017), ordered by the target, then year. (Table adapted from Ref. ${ }^{81}$ )........................... 26

Table 2.1: Sequence library templates for DZA and MR libraries and their respective

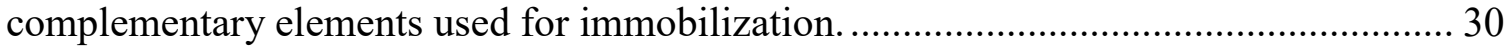

Table 2.2: Full-length candidate sequences from the DZA templated library.................. 31

Table 2.3: Full-length candidate sequences from the random aptamer templated library 32

Table 2.4: Composition of buffers used in chapter 2

Table 2.5: Composition and distribution of G-quadruplex forming G-rich sequence

DZA3, as predicted by QGRS Mapper 46

Table 2.6: Composition and distribution of Q-quadruplex forming G-rich sequence

DZA5, as predicted by QGRS Mapper 46

Table 2.7: Composition and distribution of G-quadruplex forming G-rich sequence

DZA7, as predicted by QGRS Mapper 47

Table 2.8: Melting temperatures for oligonucleotide sequences 57

Table 3.1: Modified DZA3 aptamers used for the design of signalling probes. 82

Table 3.2: Modified 3MR sequences used for the design of hybridized signalling probes 
Table 3.3: Modified DZA capture probes used for the design of hybridized signalling probes.

Table 3.4: Modified 3MR capture probes used for the design of hybridized signalling probes.

Table 3.5: Summary of the fluorescence recovery obtained when challenging structureswitching signalling probes with $10 \mathrm{nM}$ aflatoxin $\mathrm{B}$ 


\section{List of Figures}

Figure 1.1: Structures of aflatoxin $\mathrm{B}_{1}(2,3,6 \mathrm{aR}, 9 \mathrm{aS}-$ tetrahydro-4-methoxy-1H,11Hcyclopenta[c]furo[3',2':4,5]furo[2,3-h][1] benzopyran-1,11-dione) MW=312.28 g/mol, aflatoxin $\mathrm{B}_{2}(2,3,6 \mathrm{aR}, 8,9,9 \mathrm{aS}-$ hexahydro-4-methoxy-cyclopenta[c]furo[3',2':4,5]furo[2,3h][1]benzopyran-1,11-dione) MW=314.29 g/mol, aflatoxin $\mathrm{G}_{1}(7 \mathrm{aR}, 10 \mathrm{aS})-3,4,7 \mathrm{a}, 10 \mathrm{a}-$ tetrahydro-5-methoxy-1H,12H-furo[3',2':4,5]furo[2,3-h]pyrano[3,4-c][1]benzopyran1,12-dione) $\mathrm{MW}=328.28 \mathrm{~g} / \mathrm{mol}$, and aflatoxin $\mathrm{G}_{2}(7 \mathrm{aR}, 10 \mathrm{aS})-3,4,7 \mathrm{a}, 9,10,10 \mathrm{a}-\mathrm{hexahydro}-$ 5-methoxy-1H,12H-furo[3',2':4,5]furo[2,3-h]pyrano[3,4-c][1] benzopyran-1,12-dione) $\mathrm{MW}=330.29 \mathrm{~g} / \mathrm{mol}$

Figure 1.2: Capture SELEX process ${ }^{58}$ A) Capture sequence is immobilized onto solid support matrix (agarose bead) via biotin-streptavidin interaction B) Sequences contain a known central region (red) flanked by two random randomized region C) Sequences are immobilized via hybridization between the known region and immobilized capture sequence D) Elution with target is used to evoke structural changes and the release of binding sequences E) Partitioning of binding sequences and amplification...................... 19

Figure 3.1: Schematic representation of sequence template with fixed internal sequence (Red), flanking randomized regions (Blue), primer binding regions (Green), and biotinylated capture probe.

Figure 3.2: Graphical representation of common G-quadruplex topologies A) parallel Gquadruplex with anti conformation of the guanine glycosyl bonds B) antiparallel Gquadruplex adopting the chair conformation C) antiparallel G-quadruplex structure 
containing a diagonal loop. Both $\mathrm{B} \& \mathrm{C}$ contain anti/syn alternating conformation of the glycosyl bonds. 34

Figure 3.3: Chemical structures representing the anti and syn glycosidic bond angles. . 37 Figure 3.4: Graphical representation of electrophoretic mobility shift assay performed for aptamer candidates. Lane 1: Fluorescently labelled capture probe (CP) control. Lane 2: Hybridized aptamer and fluorescent capture probe. Lane 3: Hybridized complex challenged with aflatoxins causes disassembly of hybridized Apt/CP. The result is a decrease in top band intensity and increase in bottom (CP) band intensity. 49 Figure 3.5: EMSA results from preliminary DZA aptamer candidate screening for total aflatoxin mixture. Red bars represent bottom CP band intensity. The blue line represents the change in top $\mathrm{CP}+\mathrm{Apt}$ band intensity. 50

Figure 3.6: EMSA results from preliminary 3MR aptamer candidate screening for total aflatoxin mixture. Red bars represent bottom $\mathrm{CP}$ band intensity. The blue line represents the change in top $\mathrm{CP}+\mathrm{Apt}$ band intensity. 51

Figure 3.7: EMSA results from preliminary selectivity screening with counter toxins FB1, OTA, ZON, and DON for aptamers DZA3 (A), DZA5 (B), DZA7 (C). Red bars represent bottom $\mathrm{CP}$ band intensity. The blue line represents the change in top $\mathrm{CP}+\mathrm{Apt}$ band intensity. 53

Figure 3.8: EMSA results from preliminary selectivity screening with counter toxins FB1, OTA, ZON, and DON for aptamers 3MR2 (A), 3MR3 (B), 3MR5 (C). Red bars 
represent bottom $\mathrm{CP}$ band intensity. The blue line represents the change in top $\mathrm{CP}+\mathrm{Apt}$ band intensity.

Figure 3.9: Schematic representation of the thermal denaturation of duplexed DNA. The UV melting temperature curve demonstrates that the hyperchromic effect is proportional to the rate of denaturation. The melting temperature is taken as the midpoint in the hyperchromic transition, where the amount of ssDNA is of equal proportion to that of the dsDNA in solution 56

Figure 3.10: Secondary structure prediction for A) DZA3, B) DZA7, and C) DZA5 sequences. Image generated by UNAFold. 58

Figure 3.11: Secondary structure prediction for A) 3MR2, B) 3MR3, and C) 3MR5 sequences. Image generated by UNAFold. 59

Figure 3.12: Melting temperature curves for PS2.M G-quadruplex forming sequence (295 nm). Hyperchromicity observed at low temperatures suggests the presence of higher order structures.

Figure 3.13: Example of melting temperature profile from DZA templates (DZA3). The decrease in intensity resulting from G-quadruplex melting is masked by an overlying 
increase in absorbance caused by the hyperchromic effect. The resulting "hump" in the spectrum is not present in the $3 \mathrm{MR}$ candidates

Figure 3.14: Example of melting temperature profile random template (3MR2). The sequence does not exhibit increased absorbance at low temperatures, suggesting there is no G-quadruplex formation.

Figure 3.15: CD spectrum of parallel-stranded G-quadruplex forming PS2.M sequence in $20 \mathrm{mM}$ Tris buffer ( $\left.100 \mathrm{mM} \mathrm{NaCl}, 5 \mathrm{mM} \mathrm{KCl}, 1 \mathrm{mM} \mathrm{CaCl}_{2}, 1 \mathrm{mM} \mathrm{MgCl}_{2}\right)$, as monitored by UV-CD using an Olis Cary 17 CD spectropolarimeter.

Figure 3.16: CD spectrum of DZA aptamer candidate sequences in $20 \mathrm{mM}$ Tris buffer (100 mM NaCl, $5 \mathrm{mM} \mathrm{KCl}, 1 \mathrm{mM} \mathrm{CaCl}_{2}, 1 \mathrm{mM} \mathrm{MgCl}_{2}$ ), as monitored by UV-CD using an Olis Cary $17 \mathrm{CD}$ spectropolarimeter. 63

Figure 3.17: CD spectrum of 3MR aptamer candidate sequences in $20 \mathrm{mM}$ Tris buffer (100 mM NaCl, $5 \mathrm{mM} \mathrm{KCl}, 1 \mathrm{mM} \mathrm{CaCl}_{2}, 1 \mathrm{mM} \mathrm{MgCl}_{2}$ ), as monitored by UV-CD using an Olis Cary $17 \mathrm{CD}$ spectropolarimeter.

Figure 3.18: Successful capillary scan for DZA3 samples. Non-specific adsorption of sample onto the capillary wall is normally demonstrated by irregularly shaped fluorescence peaks along the capillary 66 Figure 3.19: Normalized fluorescence measurement for Cy5 labelled DZA3. Performed in order to ensure there is no precipitation or aggregation effect. The figure represents an MST time trace. The initial cold phase (green) shows the sample fluorescence with the laser off. The laser is switched on after the 5 second and a temperature gradient is 
established. A decrease in fluorescence is observed due to thermophoretic movement (red). After 30 seconds, the laser is turned off and the molecules diffuse back. 66

Figure 3.20: A) Binding curve for DZA3 and $\mathrm{AFB}_{1} \mathrm{~B}$ ) Binding curve for $\mathrm{DZA} 3$ and

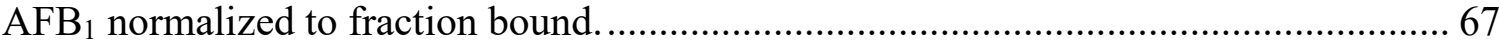

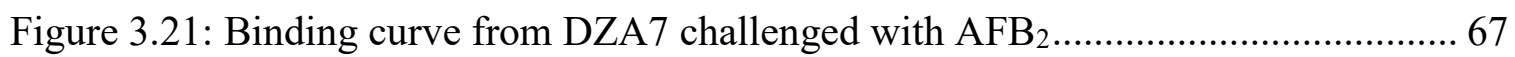

Figure 3.22: A) Binding curve for $\mathrm{DZA} 3$ and $\mathrm{AFG}_{2} \mathrm{~B}$ ) Binding curve for $\mathrm{DZA} 3$ and

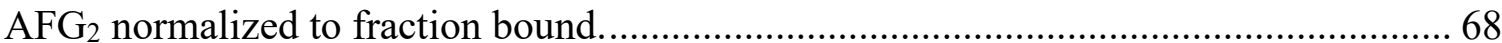

Figure 3.23: A) Binding curve for 3MR5 and $\mathrm{AFB}_{1} \mathrm{~B}$ ) Binding curve for $3 \mathrm{MR} 5$ and

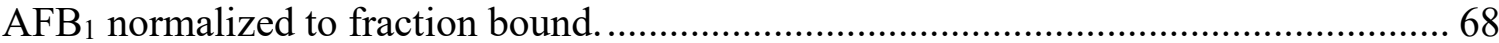

Figure 4.1: Chemical structure of dually-linked Cyanine 3 modifier.............................. 82

Figure 4.2: Chemical structure of Black hole quencher 1 deoxythymidine modifier

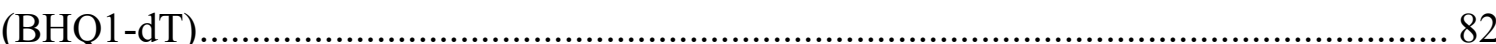

Figure 4.3: Quenching measurements for fixed DZA3_BHQ1 concentration in combination with increasing 24-nt DZCP_FAM sequence. 85

Figure 4.4: A) Quenching measurements for varying aptamer capture probe ratios using DZA3_FAM and 24-nt DZCP_BHQ1 sequences. B) spectral data resulting from quenching efficiency experiments. The error bars are represented as shadows of the same colour underlying each line in the spectrum. 86 Figure 4.5: A) Quenching measurements for varying aptamer capture probe ratios using DZA3_FAM and 12-nt DZCPnT_BHQ1 sequences. B) spectral data resulting from 
quenching efficiency experiments. The error bars are represented as shadows of the same colour underlying each line in the spectrum. 87

Figure 4.6: A) Quenching measurements for varying aptamer capture probe ratios using DZA3_FAM and 9-nt DZCP9.0_BHQ1 sequences. B) spectral data resulting from quenching efficiency experiments. The error bars are represented as shadows of the same colour underlying each line in the spectrum. 87 Figure 4.7: A) Quenching measurements for varying aptamer capture probe ratios using DZA3_FAM and 12-nt DZCPM1_BHQ1 sequences. B) spectral data resulting from quenching efficiency experiments. The error bars are represented as shadows of the same colour underlying each line in the spectrum. 88 Figure 4.8: A) Quenching measurements for varying aptamer capture probe ratios using DZA3_Cy3 and 12-nt DZCPnT_BHQ1 sequences. B) spectral data resulting from quenching efficiency experiments. The error bars are represented as shadows of the same colour underlying each line in the spectrum. 90 Figure 4.9: A) Quenching measurements for varying aptamer capture probe ratios using 3MR5_FAM and 12-nt RCPnT_BHQ1 sequences. B) spectral data resulting from quenching efficiency experiments. The error bars are represented as shadows of the same colour underlying each line in the spectrum. 91 Figure 4.10: A) Quenching measurements for varying aptamer capture probe ratios using 3MR5_Cy3 and 12-nt RCPnT_BHQ1 sequences. B) spectral data resulting from 
quenching efficiency experiments. The error bars are represented as shadows of the same colour underlying each line in the spectrum.

Figure 4.11: Schematic representation of structure-switching hybridized signalling platform for the detection of AF. Inversely modified FRET assembly (Above) Common arrangement for FRET assembly (Below).

Figure 4.12: DZA3_FAM/DZCPnT_BHQ1 quenching (37.3\%) and testing with $100 \mathrm{nM}$ $\mathrm{AFB}_{1}$. Challenging hybridized probe with equimolar target showed no significant increase in fluorescence. 97 Figure 4.13: Structure dependent fluorescence intensity decrease with the addition of 10x excess aflatoxin $\mathrm{B}_{1}$ A) DZA3_FAM resulted in a 13.6\% decrease in fluorescence B) 3MR5 FAM resulted in a 14\% decrease in fluorescence. 100 Figure 4.14: Structure dependent fluorescence intensity decrease with the addition of 10x excess aflatoxin $B_{1}$ A) DZA3_Cy3 resulted in a 42.2\% decrease in fluorescence B) 3MR5_Cy3 resulted in a $44.5 \%$ decrease in fluorescence. 


\section{List of Abbreviations}

$\mathrm{AFB}_{1}$ : Aflatoxin $\mathrm{B}_{1}$

$\mathrm{AFB}_{2}$ : Aflatoxin $\mathrm{B}_{2}$

$\mathrm{AFG}_{1}$ : Aflatoxin $\mathrm{G}_{1}$

$\mathrm{AFG}_{2}$ : Aflatoxin $\mathrm{G}_{2}$

$\mathrm{AFM}_{1}$ : Aflatoxin $\mathrm{M}_{1}$

Apt: Aptamer

AuNP: Gold nanoparticle

BHQ1: Black hole quencher 1

CD: Circular dichroism

cDNA: Complementary DNA

CP: Capture probe

CPG: Controlled pore glass

CPL: Circularly polarized light

CRM: Certified reference material

CV: Cyclic voltammetry

Cy3: Cyanine 3

DNA: Deoxyribonucleic acid

DMT: 4'4-dimethoxytritil

DON: Deoxynivalenol

ECL: Electrochemiluminescence

EIS: Electrochemical impedance spectroscopy

ELEUM: equine leukoencephalomalacia 
ELISA: Enzyme-linked immuno sorbent assay

FAM: Carboxy fluorescein

FB1: Fumonisin B1

FP: Fluorescence polarization

FRET: Förster resonance energy transfer

GO: Graphene oxide

HCC: Hepatocellular carcinoma

HPLC: High-performance liquid chromatography

HRP: Horseradish peroxidase

IARC: International Agency for Research on Cancer

LDR: Linear dynamic range

LOD: Limit of detection

MST: Microscale thermophoresis

NIV: Nivalenol

nt: Nucleotide

OTA: Ochratoxin A

PAGE: Polyacrylamide gel electrophoresis

PCR: Polymerase chain reaction

Q-dots: Quantum dots

QE: Quenching efficiency

RET: Resonance energy transfer

RT-qPCR: Real-time quantitative polymerase chain reaction

SPCE: Screen printed carbon electrode 
SPR: Surface plasmon resonance

TAMRA: Carboxytetramethylrhodamine

TEAA: Triethylammonium acetate

TFA: Trifluoroacetic acid

Tris: Tris(hydroxymethyl)aminomethane

TLC: Thin layer chromatography

ZON: Zearalenone 


\section{Chapter: Introduction}

\subsection{Mycotoxins}

Mycotoxins are a diverse group of low molecular weight compounds produced by toxigenic fungi belonging to numerous fungal genera including; Aspergillus, Penicillium, Fusarium, Alternaria, and Claviceps.${ }^{1,2}$ Mycotoxins differ greatly in terms of their chemical structure and their biosynthetic origins. As a rule, however, mycotoxins are produced by microfungi (moulds) and are toxic in low doses to humans and other vertebrates. They are not to be confused with toxins that are present in the fruiting bodies of mushrooms, for example, amatoxin and orellanine, or other small fungal metabolites such as antibiotics, pigments, and phytotoxins. ${ }^{3}$ Mycotoxins commonly contaminate food commodities such as wheat, maize, barley, groundnuts, spices, and dried fruit among others, and as a result are ingested unintentionally. ${ }^{2,4}$ Secondary exposure to humans can occur through the and metabolism of mycotoxins in livestock, followed by their accumulation in meat, milk, and eggs, for example. Mycotoxin ingestion can cause wideranging acute and chronic toxicities. Many mycotoxins exhibit overlapping toxicities regardless of their fungal origins. Symptoms resulting from mycotoxin exposure are referred to as mycotoxicosis, can vary depending on the type of toxin, the rate of exposure, the age, and the health of an individual. ${ }^{5}$ Since mycotoxicosis is abiotic in nature and does not involve the fungi themselves, diseases that arise from mycotoxin exposure are non-transmissible and are not treatable with drugs, and antibiotics. ${ }^{6}$ The ill effects of mould infested foodstuffs were experienced long before mycotoxins were identified as the culprit. In the Middle Ages, an illness known then as St. Anthony's fire was caused due to the chronic ingestion of ergot alkaloids produced in the sclerotia of 
certain species of Claviceps. The disease was defined by its hallucinations, intense burning sensations, convulsions, and gangrenous symptoms. ${ }^{7,8}$ The understanding that foodborne fungal metabolites were responsible for major epidemics in humans and domesticated animals became apparent in the 1960's. The following 15 years were referred to as the mycotoxin gold rush due to an upsurge in mycotoxin research. ${ }^{7}$

The discovery of most mycotoxins was preceded by acute poisoning epidemics in animals. Cases of equine leukoencephalomalacia (ELEUM) for example, arose in the early 1890's due to the consumption of fumonisin in mouldy corn. The disease was loosely coined the "corn stalk" disease due to its association with that type of feed alone. ${ }^{9}$ It wasn't until 1988 that Marasas et al. provided experimental evidence that fumonisin B1 produced by Fusarium verticillioides (also referred to as Fusarium moniliforme) caused ELEUM. ${ }^{9,10}$ Similarly, aflatoxin was discovered following an incident known as Turkey $\mathrm{X}$ disease. Roughly 100,000 poults died from an unknown disease in the south of England. Following its spread to farmed duck and pheasants, it was discovered that the disease was associated with the feeding of Aspergillus flavus contaminated groundnut meal from Brazil. ${ }^{11}$ Today, the mycotoxin classes that are of highest public and agroeconomic importance are: Aflatoxins, ochratoxins, fumonisins, trichothecenes, and zearalenone. 
Table 1.1: Important mycotoxin classes, their fungal producers, and commonly contaminated foodstuffs ${ }^{5}$

\begin{tabular}{|c|c|c|}
\hline Mycotoxin Class & Principle Fungal Producers & Contaminated Foodstuffs \\
\hline Aflatoxins & $\begin{array}{l}\text { A. flavus } \\
\text { A. parasiticus }\end{array}$ & $\begin{array}{l}\text { Groundnuts, maize, } \\
\text { cottonseed }\end{array}$ \\
\hline Ochratoxins & $\begin{array}{l}\text { A. ochraceus } \\
\text { P. verrucosum }\end{array}$ & $\begin{array}{l}\text { Beans, chickpeas, pecans, } \\
\text { pistachios, green coffee } \\
\text { beans, cereals }\end{array}$ \\
\hline Fumonisins & $\begin{array}{l}F \text {. verticillioides } \\
F . \text { proliferatum }\end{array}$ & Maize \\
\hline $\begin{array}{l}\text { Trichothecenes } \\
\text { (DON, NIV) }\end{array}$ & $\begin{array}{l}\text { F. graminareum } \\
\text { F. culmorum }\end{array}$ & Wheat, barley, triticale \\
\hline Zearalenone & $\begin{array}{l}\text { F. graminareum } \\
\text { F. colmorum }\end{array}$ & Wheat, barley, triticale \\
\hline
\end{tabular}

There are three main factors in determining which mycotoxins are of foremost importance; the nature of their fungal producers, the substrates which they colonize, and the potency of the toxins produced. ${ }^{12}$ Hundreds of mycotoxins have been identified however a large number them are of little concern due to the fact that they are produced by fungi that do not typically colonize food or animal feed. Several toxigenic Penicillium species that are prominent in soils do not contribute significant contamination to crops. On the other hand, some toxigenic fungi that contaminate crops produce so little toxin under normal conditions that they do not present a real hazard. Examples of these include cyclopiazonic acid produced by Aspergillus flavus, and tenuazonic acid produced by Alternaria species. A number of these classes of toxins are associated with visible fungal spoilage resulting in the rejection of produce. ${ }^{5}$ Finally, some toxins do not exhibit substantial toxicity when exposure occurs naturally. Sterigmatocystin is a mycotoxin produced by Aspergillus versicolor, a somewhat common fungus that is found in stored commodities. The structure and the bioactivity are similar to those of $\mathrm{AFB}_{1}$, however, its 
lack of solubility in water and acids results in a toxicity that is over 100 -fold less toxic than $\mathrm{AFB}_{1},{ }^{5,7}$

Mycotoxin contamination can occur during virtually all stages of food production including the pre-harvest, harvest, drying, and storage. This often depends on the nature of the fungi colonizing the crop. Pathogenic fungi such as Fusarium graminearium are virulent and cause diseases like head blight when they colonize crops. Aspergillus flavus on the other hand is said to be saprophytic in nature. Contamination typically occurs during plant development when crops are compromised by birds, mammals, insects, or climatic stressors. This predisposes the crops to mycotoxin contamination after harvest. ${ }^{12,13}$ Other fungi such as Aspergillus ochraceous are found in the soil or on decaying plant matter. The contamination of developing kernels can lead to contamination postharvest. ${ }^{12}$ While multiple bacterial and fungal metabolites are virulence factors and play a functional role in the progression of host colonization, the overall role of mycotoxin production and the growth and development of fungi is said to be largely unknown. ${ }^{7}$ The fact that that mycotoxin production is highly regulated and is resource consuming, supports that these compounds play some role in the biology of the organisms. ${ }^{14}$

\subsubsection{Aflatoxins}

Aflatoxins are the most thoroughly studied class of mycotoxins. They belong to a class of fungal metabolites that were first discovered in the 1960's following a severe livestock epidemic known as Turkey "X" Disease. ${ }^{15}$ They have since become one of the most studied classes of mycotoxins. ${ }^{2}$ They are of great concern from both a public health and agro-economic standpoint. Aflatoxins are produced primarily by Aspergillus flavus 
and Aspergillus parasiticus fungi. The four major types of aflatoxins are aflatoxin B1, B2, G1, and G2, named for their blue/green fluorescence. Aspergillus flavus is primarily responsible for the production of aflatoxins B1, and B2 while Aspergillus parasiticus, on the other hand, produce aflatoxins B1, B2, G1, and G2. Aflatoxins are difuranocoumarin compounds synthesized through the polyketide pathway. Their production is highly regulated. Genetic studies indicate that there are 17 genes responsible for 12 enzymatic conversions in the aflatoxin/sterigmatocystin biosynthetic pathway. ${ }^{14}$

Aflatoxin producing fungi are primarily found in tropical and sub-tropical climates. Aspergillus flavus and Aspergillus parasiticus primarily colonize corn, cottonseed, peanuts and tree nuts. ${ }^{5}$ The proliferation of aflatoxin producing fungi occurs in distinct stages which typically coincide with growth phases of the crop. Under optimal conditions, fungal proliferation can also occur during storage. ${ }^{16,17}$ 


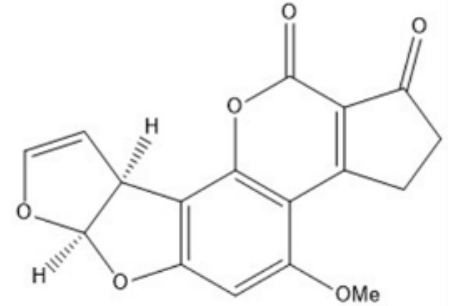

$\mathrm{AFB}_{1}$

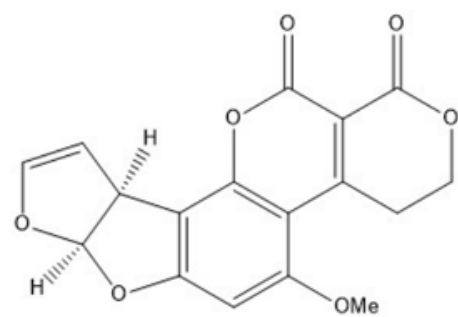

$\mathbf{A F G}_{1}$

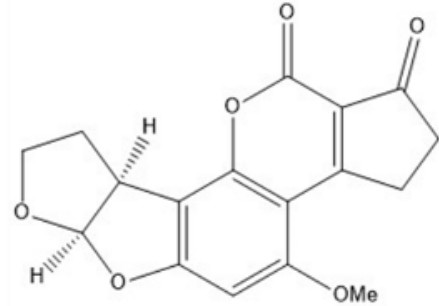

$\mathrm{AFB}_{2}$

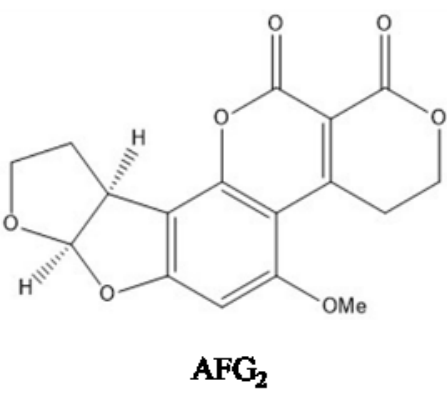

Figure 1.1: Structures of aflatoxin $B_{1}(2,3,6 \mathrm{aR}, 9 \mathrm{aS}-$ tetrahydro-4-methoxy-1H,11Hcyclopenta[c]furo[3',2':4,5] furo[2,3-h] [1] benzopyran-1,11-dione) $M W=312.28 \mathrm{~g} / \mathrm{mol}$, aflatoxin $B_{2}$

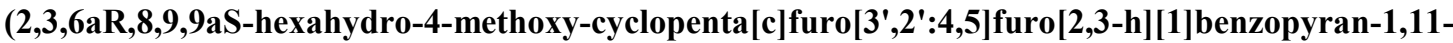
dione) $M W=314.29 \mathrm{~g} / \mathrm{mol}$, aflatoxin $\mathrm{G}_{1}(7 \mathrm{aR}, 10 \mathrm{aS})-3,4,7 \mathrm{a}, 10 \mathrm{a}-$ tetrahydro-5-methoxy-1H,12Hfuro[3',2':4,5] furo[2,3-h]pyrano[3,4-c][1] benzopyran-1,12-dione) $M W=328.28 \mathrm{~g} / \mathrm{mol}$, and aflatoxin $\mathrm{G}_{2}$ (7aR,10aS)-3,4,7a,9,10,10a-hexahydro-5-methoxy-1H,12H-furo[3', $\left.2^{\prime}: 4,5\right]$ furo[2,3-h]pyrano[3,4c][1]benzopyran-1,12-dione) $\mathrm{MW}=330.29 \mathrm{~g} / \mathrm{mol}$.

Aflatoxins have been shown to be extremely potent carcinogens in several species. ${ }^{5}$ They exhibit genotoxic effects. $\mathrm{AFB}_{1}$ is bioactivated by liver microsomes CYP1A2, 3A4, and 2A6. Aflatoxin $\mathrm{M}_{1}$ is an oxidative metabolite of $\mathrm{AFB}_{1}$. It is the primary unconjugated metabolite excreted in urine or in milk. It is formed via the oxidation of $\mathrm{AFB}_{1}$ by microsomal cytochrome $\mathrm{P} 4501 \mathrm{~A} 2 .{ }^{18} \mathrm{AFB}_{1}$ is also converted to the highly reactive $\mathrm{AFB}_{1}$ endo-, and exo-8,9-epoxides intermediates which can bind to proteins and DNA. The $\mathrm{AFB}_{1}-8,9$-epoxide intercalates readily into double stranded DNA resulting in DNA adduct formation. The major DNA adducts are $\mathrm{AFB}_{1}-\mathrm{N}^{7}$-guanine, and $\mathrm{AFB}_{1}$-formamidopyrimidine ( $\mathrm{AFB}_{1}$-FAPY). ${ }^{19,20}$ Hepatocellular carcinoma in aflatoxin- 
endemic regions has been associated with a specific mutation that results in the inactivation of the p53 tumor suppressor gene. The predominant mutations induced by $\mathrm{AFB}_{1}$ exposure are a result of a $\mathrm{G}$ to $\mathrm{T}$ transversion mutation at codon 249 , causing an Arginine to Serine point mutation. ${ }^{5,20}$ Other procarcinogenic effects include sister chromatid exchanges, and chromosomal aberrations. Epidemiological research indicates aflatoxin has a strong causal association with liver cancer in populations with elevated exposure. Higher risks of hepatocellular carcinoma (HCC) occur in individuals that are chronically infected with hepatitis B virus. ${ }^{6}$ As such, aflatoxin is classified as group 1 carcinogen. ${ }^{21}$ Early life exposure has also been associated with stunting (impairment of child growth). ${ }^{22}$ Early symptoms of acute toxicity include weight loss, systemic immune suppression. Symptoms of high-level exposure can progress to hemorrhagic necrosis of the liver and bile duct proliferation, jaundice, and hepatic failure resulting in death. ${ }^{22}$

Of the common biomarkers for effective dose of aflatoxins, DNA adducts are of primary interest due to the fact that they represent damage made to a critical cellular macromolecular target. ${ }^{18}$ Detoxification of the epoxide and $\mathrm{AFM}_{1}$ in mammalian tissues typically occurs via conjugation by glutathione, catalysed by glutathione S-transferase. The most reliable biomarkers are excreted in urine of faecal matter and provide the establishment of links between dietary exposure, accumulation, and excretion. ${ }^{18}$ The aflatoxin $\mathrm{B}_{1}-\mathrm{N}^{7}$-guanine adduct is the most reliable urinary biomarker for aflatoxin exposure, however it provides a measure of exposure over relatively short periods (24 $48 \mathrm{~h}) . \mathrm{AFB}_{1}-\mathrm{N}^{7}$-guanine is a better measure of acute exposure. The longer half-life of $\mathrm{AFB}_{1}$-albumin provides a measure of human exposure over longer periods. ${ }^{18}$ 
Table 1.2: Maximum tolerable levels for total aflatoxin contaminants in human food ${ }^{23}$

\begin{tabular}{|c|c|}
\hline Country or Region & $\begin{array}{c}\text { Total allowable level of aflatoxins in human } \\
\text { food }(\mu \mathrm{g} / \mathrm{Kg})\end{array}$ \\
\hline Australia & 5 (all foods), 10 (groundnuts) \\
\hline Canada & 15 (nuts, groundnuts) $^{\mathrm{a}}$ \\
\hline U.S. & 20 (all foods), 0.5 (milk) \\
\hline European Union & $\begin{array}{c}4 \text { (cereals and cereal products), } 10 \text { (maize), or } \\
15 \text { (nuts, groundnuts) }\end{array}$ \\
\hline
\end{tabular}

${ }^{a}$ Specified tolerance by the Canadian Food and Drug Regulations. There are currently no regulations established in Canada.

\subsubsection{Impact of mycotoxins and risk management}

The economic impacts that are a result of mycotoxin contamination can be assessed based on two major categories. The first is the public health impact associated with the adverse effects of mycotoxin exposure. The second is the direct effect on markets that is caused by the loss of revenue due to contaminated food and feed.

Mycotoxin regulation in developing countries poses a greater challenge. Regions which rely heavily on the small-scale farming of single food commodities such as wheat and maize are often at risk for high mycotoxin exposure. Local distribution of food is often untested and unregulated. Other scenarios include those in which the highest quality produce is directed to commercial market while the worst quality food is either kept for local consumption of diverted to poorer sectors. ${ }^{5,8}$ Regions that are affected by high levels of mycotoxin exposure, often struggle with sufficient food production and undernourishment and therefore are unable to prioritize food safety standards. High dietary uptake of mycotoxins is also greatly influenced by the lack of dietary diversity. Moreover, the severity of mycotoxicosis is often amplified by factors such as infectious 
diseases, malnutrition, and vitamin deficiencies. Therefore, it is often the most vulnerable populations that are affected. The encouragement of low-cost strategies at the household level in good agricultural and storage practices, as well as processing techniques such as grain sorting have had a positive effect in high risk populations. Due to the overreliance of easily contaminated crops, dietary diversification has also been considered. These have become the most feasible option for effective exposure reduction in low-income countries. However, they have yet to incorporated into a sustainable intervention method that can attain large populations ${ }^{5,23}$

In contrast, developed countries have the resources and methodologies to uphold strict regulatory control. Human health effects are said to be grossly negligible. The effects of mycotoxin contamination result in greater economic losses for food and agricultural industries. ${ }^{13,24}$ The primary ways in which mycotoxins affect markets is the loss of value of traded commodities and decreased market supply. Mycotoxin contamination affects both regional and international markets. ${ }^{23}$ Estimated combined losses caused by the mycotoxins of greatest agro-economic importance are estimated to be between 0.5 and 1.5 billion dollars per year in the U.S. alone. Almost all the losses were from maize and groundnuts. In comparison, Thailand, the Philippines, and Indonesia experience similar losses from aflatoxin alone. ${ }^{5}$ In developed countries, it is easier to assess impacts of mycotoxins as well as the cost and feasibility of interventions because the impacts are typically limited to market. Currently, there is no harmonized maximum tolerable level. Table 1.2 provides examples of maximum tolerable levels of aflatoxins in specific food commodities. Mycotoxins are regulated in over 77 countries. Many regulations vary based on the type of mycotoxin, the contaminated food 
commodity, and the allowed limit. .It has been estimated however that the harmonization of standards would greatly influence the economic burden the world's top maize and groundnut exporting countries. ${ }^{23}$

\subsubsection{Methods of detection}

Due to the large public health and economic impacts of mycotoxin contamination, the detection of mycotoxins in foodstuffs is of immense importance. Their presence in low concentrations demands analytical methods with high sensitivity and specificity. ${ }^{19}$ Analytical methods that are used by enforcement laboratories for the implementation of legislation must be subject to validation procedures. ${ }^{25}$ Performance standards for these methods have been established at a national and international level by European Regulation and The Association of Official Analytical Chemists International for example. $^{25}$

Methods that are routinely used for mycotoxin analysis include thin-layer chromatography (TLC), high-performance liquid chromatography (HPLC), and enzymelinked immuno sorbent assay (ELISA). ELISAs are intended for use as fast screening methods for mycotoxins such as aflatoxins, fumonisins, ochratoxin A, zearalenone, and trichothecenes. ${ }^{25,26}$ They offer high sample throughput at comparatively low costs. The use of high sample throughput screening methods was estimated by the EU to reduce analysis costs by roughly a third in comparison to the use of only confirmatory type methods. ${ }^{25}$ ELISAs are susceptible to matrix dependence which can alter recognition events. As such, they are more susceptible to false negative results. Cross-reactivity can also lead to false positive results. Confirmatory methods such as HPLC-type procedures 
are therefore necessary for positive ELISA results. ${ }^{27}$ Other innovative screening methods that have been developed include lateral flow assays and immunochemistry based SPR.

Sample preparation typically entails the extraction of mycotoxins followed by immunoaffinity chromatography clean-up step. Immunoaffinity column based clean up has helped increase the performance of chromatographic techniques significantly given they provide very clean sample extracts. They have also been used for automated cleanup steps. ${ }^{25}$ Mycotoxins are varied in terms of their chemical and physicochemical properties. Specific extraction, clean-up, and detection methods are required. Many detection methods target either individual mycotoxins of mycotoxin families. The need for multi-detection of mycotoxins is apparent. In recent years increasing work has been done to develop analytical methods that simultaneously detect different classes of mycotoxins. The use of LC-MS/MS is becoming the leading method for multi-toxin detection. It was first derived from the field of mycology, in which mould species are identified based on their metabolite profiles. ${ }^{28}$ This method was greatly encouraged by the discovery of the co-occurrence of mycotoxins, and their potential synergistic effects on toxicity. The main disadvantage of multi-toxin detection is the sheer diversity of mycotoxins and their chemical properties. Multi-toxin analysis still requires the selection of procedure (extraction solvents and mobile phase) to facilitate the detection of some mycotoxins while hindering the detection of other analytes. ${ }^{28}$ Considerable progress is being made for the simultaneous determination and identification of large numbers of toxins. The use of proper certified reference materials (CRM) in analytical laboratories is a key requirement in the development of multi-toxin LC-MS/MS. 
Aflatoxins, for example, can be extracted in polar protic solvents. The extraction of aflatoxins for immunochemistry techniques is generally performed with a methanolwater mixture to avoid negative effects of the solvent on antibodies. Post column derivatization techniques such as bromination and irradiation by UV light, and the use of monoclonal and polyclonal antibodies for a number of mycotoxins (aflatoxins, Ochratoxin A, fumonisins, zearalenone, and deoxynivalenol) in immunoaffinity columnbased clean-up procedures prior to HPLC or TLC have greatly improved the performance of detection methods. The most frequently used confirmatory method for aflatoxin determination is still liquid chromatography with fluorescence detection (LC-FLD). ${ }^{27}$ All four analogues can be analysed within an individual assay. Enforcement laboratories are required to maintain reliable calibration conditions and certified reference materials for aflatoxins.

Rapid screening methods are crucial for the high throughput and cost-effective screening of foodstuffs. Important criteria for any promising assay include analytical validity, reproducibility, specificity, and stability. Currently, the standard for rapid screening methods for aflatoxins is immunochemical techniques. The use of synthetic molecular recognition elements in potential screening methods is however increasing. ${ }^{6}$ The incorporation of aptamers into existing immunochemical methods as well as the development of novel sensing platforms are a promising tool for mycotoxin screening. Moreover, the incorporation of currently used immuno-chromatographic clean-up steps can help reduce limitations such as matrix effects on biorecognition events. 


\subsection{Aptamers}

Through the discovery of a growing number of functional noncoding RNAprotein complexes, ribozymes, and their associated catalytic properties, nucleic acids were re-examined in terms of their potential capabilities. These discoveries promoted a reassessment of their biological potential and promoted further research on their possible uses. ${ }^{29}$ Prior to this, DNA or RNA was primarily recognized as information-bearing macromolecules. That is to say, their roles in gene coding, storing, processing and storing of genetic information were better known. Tuerk \& Gold's pivotal research described a generalizable in vitro technique that was used to selectively amplify an RNA pool to favour the binding of T4 DNA polymerase. They described an in vitro selection or evolution process that was referred to as SELEX (Systematic Evolution of Ligands by Exponential enrichment). Concurrently, the term aptamer was coined by Ellington \& Szostak to describe the nucleic acids that were the product of this evolutionary process. ${ }^{30,31}$ The term was derived from the Latin word aptus meaning "fitted" or "connected". Their research described a short oligonucleotide sequence that bound a small organic dye. Through this, it was demonstrated that SELEX could be applied to generate high-affinity oligonucleotide sequences capable of binding to a vast array of target molecules.

By definition, aptamers are short single stranded oligonucleotides; DNA or RNA, which have the capability of binding specific target molecules with high selectivity and high affinity. ${ }^{32,33}$ SELEX can be adapted for the selection of aptamers for a multitude of targets. Aptamers have been reported for targets including cells, proteins, ions, and small molecules. Small molecules $(<900.0 \mathrm{~g} / \mathrm{mol})$ include toxins, antibiotics, biomarkers, 
drugs, and heavy metals for example. ${ }^{34,35}$

While previously believed to be generally unstructured, in solution, aptamers adopt thermodynamically stable 3-dimensional structures. Many alternate structures have been identified including right and left-handed (Z-form) double helices, hairpins, junctions, pseudoknots, i-motifs, and G-quadruplexes among others. ${ }^{36,37}$ These ordered structures can be governed by Hoogsteen and Watson-Crick hydrogen bonding, electrostatic forces, hydrophobic effects, and the stacking or intercalation of planar moieties. Structural analyses have suggested that aptamer-ligand interactions are strongly dependent on molecular weight, the degree of rotatable bonds, and the number of H-bond acceptors and donors. A study by Mckeague $e t$ al. (2015) reported the selection data of aptamers grouped by target types from 1990 to 2013. The assembly of data indicated small molecule targets, in general, result in lower-affinity aptamers than those selected for larger ligands, such as proteins. This was further supported by a study by Carothers $e t$ al. (2010). Nonetheless, only $25 \%$ of aptamers selected within that same period were for small molecule targets. ${ }^{35,38}$

Aptamers are very similar to antibodies in both form and function. Aptamers however, provide a number of inherent advantages. For instance, aptamers are selected through an in vitro process which allows a higher degree of freedom when defining selection conditions. In contrast, antibodies are produced in vivo and are therefore limited to conditions close to physiological ${ }^{33}$. Moreover, targets that do not elicit an immunogenic response, or those that are highly toxic can provide more difficulty in the production of antibodies. The chemical synthesis of aptamers via automated chemical solid-phase synthesis also provides several benefits. Aptamer sequences can be 
synthesized in large scale with high accuracy and little batch-to-batch variation. ${ }^{39}$ Ease of modification both pre- and post-selection also greatly benefit the potential applications for aptamers. For example, reporter modified aptamers have become a useful tool for in vivo and in vitro imaging analysis due to their small size $(<30 \mathrm{kDa}) .{ }^{40}$ Moreover, one of the essential properties of aptamers is their inherent ability to hybridize specifically to their complementary sequences; an aspect that can be leveraged in biotechnological processes. Aptamers are not without disadvantages, however. They are rapidly degraded by nucleases making them susceptible to digestion in blood. Chemical modifications have been developed to increase their resistance towards digestion. Moreover, there are only four natural nucleobases in comparison the 20 amino acids. By default, aptamers have less chemical diversity.

Antibodies have been the standard for bio recognition elements in sensing platforms since the 1970s. Their use in biochemical diagnostic assays such enzymelinked immunoassays (ELISA) have been widespread. Aptamers have proven to be suitable alternatives for many pre-existing antibody-based bio analysis applications, and have been incorporated into ELISA-like assays, and lateral flow assays. ${ }^{41}$ The stability of aptamers provide further benefits for long-term storage and transport, making them suitable alternatives for more robust applications such as on-site testing. ${ }^{42} \mathrm{~A}$ large focus has been placed on aptamers for analytical purposes however, a substantial number of aptamers have developed for therapeutic purposes. Pegaptanib also marketed as Macugen by Pfizer and Eyetech is an anti-angiogenic aptamer based therapeutic for macular degeneration that was approved for use by the U.S. FDA in $2004 .^{43,44}$ 


\subsubsection{Systematic Evolution of Ligands by Exponential Enrichment (SELEX)}

The SELEX technique was first reported by Tuerk \& Gold (1990) shortly after necessary advances in both polymerase chain reaction, and oligonucleotide synthesis was achieved. ${ }^{45,46}$ The SELEX process allows the simultaneous screening of large combinatorial libraries $\left(10^{15}-10^{18}\right.$ sequences $)$ containing chemically synthesized oligonucleotides. The diversity of sequences allows them to be subject to evolutionary pressures that are imposed on them by experimental conditions. ${ }^{32,47}$ The selection process works on the basis that the large sequence diversity and the structural complexity of the pool can yield a small number of sequences with the capacity to bind the target. Since aptamers are chemically synthesized and selected through an entirely in vitro process, aptamers are well suited for high-throughput selection. ${ }^{48}$

The principles of SELEX can be applied to the selection of aptamers for all desired targets and can be tailored for downstream applications. Many adaptations have been made to the original SELEX protocol, and a number of specialized SELEX methods have been produced. The iterative process occurs over several rounds. In general, SELEX is composed of three steps; selection, partition, and enzymatic amplification. ${ }^{34,49}$ The initial library of sequences (pool) is typically synthesized with a random central region (30 80-nt) flanked by primer binding regions necessary for PCR amplification. Firstly, the library is incubated with the target of interest. Washing steps are employed to rid the pool of any nonbinding sequences. ${ }^{49} \mathrm{Next}$, the partitioning step involves the separation of binding and nonbinding sequences. Partitioning methods differ depending on the target type. Protein and other large targets are often partitioned without the use of chemical 
modification. Partition methods for these types of targets can include simple and rapid membrane filtration, centrifugation, flow cytometry, and capillary electrophoresis. ${ }^{50-53} \mathrm{In}$ contrast, small molecule SELEX frequently requires the immobilisation of the target onto a solid support. Common solid support matrices include magnetic, acrylic, and sepharose beads. Once partitioned, binding sequences are isolated and amplified via PCR in order to yield an enriched aptamer pool. The enriched sequence pool can then undergo further, more stringent selection rounds, to further enrich high-affinity binders. Counter selection steps are often included to eliminate sequences that display affinity towards the solid support or structural analogues of the target. At the end of the selection process, the enriched library is cloned, sequenced, and characterised. ${ }^{49,54,55}$

\subsubsection{Capture SELEX}

Small molecule-DNA complexation generally does not result in a sufficient mass change to allow partitioning by mass- or size-based partition methods. Wash-based methods are the most common alternative. These, however, require that either the target or the oligonucleotide library be immobilized. ${ }^{34}$ Coupling chemistry is dependent on the presence of certain functional groups. ${ }^{55}$ As previously mentioned, the steric complementarity and the number of potential hydrogen bond acceptors and donors in a molecule are likely to be important factors for the affinity of an aptamer. ${ }^{38}$ The limited numbers of functional groups that are suitable for coupling may also be potential epitopes for aptamer binding. It has been reported that the immobilization of small molecule targets can result in the selection of aptamers that bind chemically modified target with a 
higher affinity. ${ }^{56}$ Moreover, selection conditions are not always conducive to downstream applications, particularly if they require the aptamer to bind target free in solution.

The design of a novel aptamer pool by Nutiu and Li (2005) enabled the immobilization of oligonucleotides sequences instead of the target molecule. The initial intent of immobilizing the pool was to allow for the selection of unmodified aptamers that could be immediately incorporated into effective signalling probes. ${ }^{57}$ The immobilisation of the sequence library encourages the selection of aptamers with structure-switching ability. In contrast to generic SELEX, the specialized DNA library is designed with a central fixedsequence domain flanked by two random binding regions and subsequent primer binding regions. The fixed central domain is designed to hybridize an antisense sequence that is biotinylated at the 5' terminus. When incubated with the target, the immobilized state of the DNA is disrupted upon DNA-target complex formation. Binding sequences can, 
therefore, be partitioned from the nonbinding bead-bound sequences. The modified SELEX process was later referred to as capture-SELEX. ${ }^{57,58}$

Figure 1.2 presents a graphical representation of the capture-SELEX process.

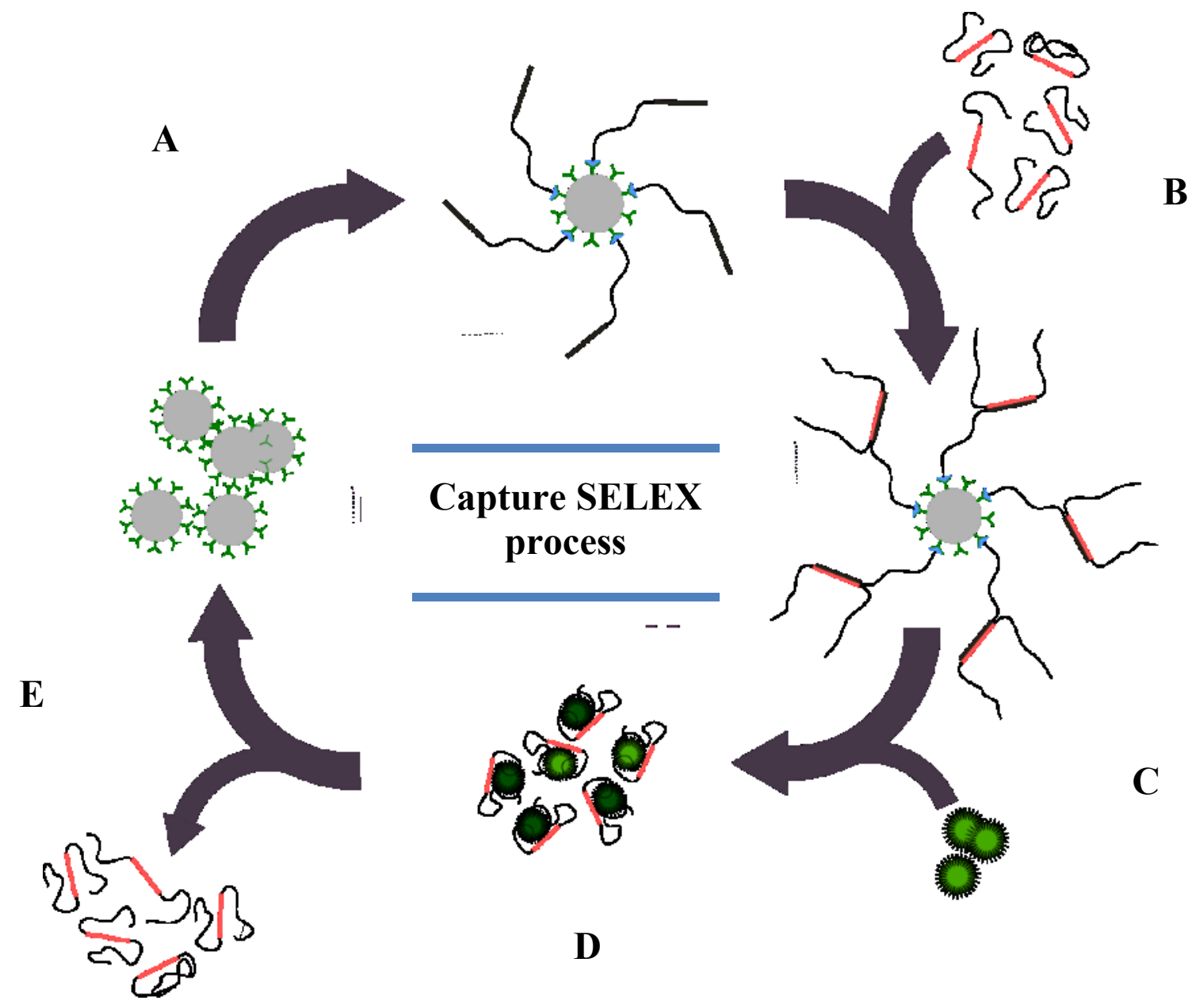

Figure 1.2: Capture SELEX process ${ }^{58}$ A) Capture sequence is immobilized onto solid support matrix (agarose bead) via biotin-streptavidin interaction B) Sequences contain a known central region (red) flanked by two random randomized region C) Sequences are immobilized via hybridization between the known region and immobilized capture sequence D) Elution with target is used to evoke structural changes and the release of binding sequences E) Partitioning of binding sequences and amplification.

Capture-SELEX has been used for a number of applications. Nutiu and Li first reported the selection of structure switching aptamers for ATP and GTP. The aptamers 
were incorporated into a fluorescent signalling probe. Another example by Soo Oh et al. used capture-SELEX in the development of a shape-changing DNA nano structure able to release a "cargo" molecule upon ligand binding. ${ }^{59}$ More importantly, the capture SELEX methodology is tailored to small molecule targets. It has permitted further advancements in the development of high-affinity aptamers that are not suitable for immobilization on a solid support, for example, aminoglycoside antibiotics. ${ }^{58}$

\subsubsection{Measuring aptamer affinity}

The capability of an aptamer to bind a cognate ligand with high affinity is typically defined by its dissociation constant, $\mathrm{K}_{\mathrm{d}}$. The dissociation constant describes an equilibrium binding association for aptamer-target complex with a 1:1 stoichiometry. The binding equilibrium is described as:

$$
\begin{gathered}
A+T \rightleftharpoons C \\
{[A] \cdot[T] \cdot k_{o n}=[C] \cdot k_{o f f}}
\end{gathered}
$$

Where A represents the aptamer, $\mathrm{T}$ is the target and $\mathrm{C}$ are the aptamer-target complex. The $\mathrm{K}_{\mathrm{d}}$ is a measure of the propensity for an aptamer-target complex (C) to dissociate reversibly into its individual constituents. The equilibrium dissociation constant can be described using both thermodynamic and kinetic definition: ${ }^{60}$

$$
\begin{gathered}
K_{d}=\frac{[\mathrm{A}][\mathrm{T}]}{[\mathrm{C}]} \\
K_{d}=\frac{k_{o f f}}{k_{o n}}
\end{gathered}
$$

The $\mathrm{K}_{\mathrm{d}}$ is inversely proportional to the affinity of the aptamer. A smaller dissociation constant relates to a higher binding affinity. Generally, affinity experiments are performed by titrating a constant concentration of aptamer with an increasing 
concentration of target. Doing the opposite is also possible. Using the thermodynamic definition, the fraction of aptamer bound can be given by:

$$
f_{a}=\frac{[T]}{K_{d}+[T]}
$$

$K_{d}$ values are most often determined through the use of binding isotherms. The $K_{d}$ can be estimated using nonlinear regression analysis. ${ }^{60}$ The $\mathrm{K}_{\mathrm{s}}$ is taken as the concentration of target when $f_{a}=0.5$.

There are a number of reported methods for determining dissociation constants. Equilibrium dialysis, gel electrophoresis, fluorescence anisotropy, and surface plasmon resonance are all examples of techniques that have been used. The above techniques are also all examples of experiments that require to aptamer-target complex to elicit a significant structural change, or change in mass. The same limitations that small molecule targets incur in the partitioning stages of SELEX are experienced for $\mathrm{K}_{\mathrm{d}}$ determination. As a result, some of the most common separation-based $\mathrm{K}_{\mathrm{d}}$ methods are not easily tailored to small molecule targets. ${ }^{55,60}$ A number of spectroscopic methods, for instance, those that use the intrinsic fluorescence of the target, or labelled DNA can be used to characterise small molecule targets. ${ }^{61}$ Other potential methods include isothermal titration calorimetry (ITC), Real-time qPCR, and Equilibrium dialysis. ${ }^{62-64}$

Methods for determining the binding have become extremely varied and are continuously being developed and improved. One of the potential limitations for the widespread use of aptamers is the lack of standardized characterization methods ${ }^{65}$ No single method is can perfectly describe the binding capabilities of an aptamer. Moreover, careful consideration must be taken for sample conditions in which aptamers are 
characterized when comparing $\mathrm{K}_{\mathrm{d}} \mathrm{s}$. The goal of aptamer development is to select aptamers that have the highest affinity possible without necessarily taking their performance into account. A comprehensive comparison of multiple common binding assays for various ochratoxin A (OTA) aptamers was performed by Mckeague et al. (2015). The study emphasized that the apparent $K_{d}$ of an aptamer is both method and aptamer dependent. The performance of the aptamers in various assays may not reflect the results of their affinity measurements. One factor that may influence this is the inherent change in structure associated with ligand binding. A number of common techniques rely on this property. Not all aptamers illicit measurable conformational changes, however. ${ }^{55,66}$ This is one example that highlights the importance of characterizing aptamer under conditions that mimic those of the downstream applications. It has also been proposed that the development of aptamers take advantage

of multiple complementary characterization techniques. ${ }^{60,65}$

\subsubsection{Aptamers for mycotoxins}

Aptamers for mycotoxins have been selected for their application in contaminated foodstuffs. It is, therefore, the most commonly identified aflatoxin analogues that are prioritized. $\mathrm{AFB}_{1}$ is the most prominent aflatoxin, and the most toxic. It has therefore been the subject of much of the aptamer-based aflatoxin research. While not as prominent, Aflatoxin $\mathrm{M}_{1}$ is still important as a secondary contaminant in alternate food sources. When livestock is fed with contaminated meal, $\mathrm{AFB}_{1}$ can be hydroxylated by liver cytochrome $\mathrm{P} 450$ s, yielding $\mathrm{AFM}_{1}$. As a result, $\mathrm{AFM}_{1}$ can be detected in milk, eggs, and sometimes meat. Previously selected aptamers for aflatoxins were selected using a 
wash-based method with aflatoxin immobilized onto magnetic nanoparticles ${ }^{67-69}$ Table 1.3shows aptamers that have been developed in recent years.

Table 1.3: Aptamers that have been previously selected for aflatoxin targets

\begin{tabular}{|c|c|c|c|c|}
\hline Target & Aptamer & Sequence & $\begin{array}{l}\text { Dissociation } \\
\text { constant }\left(K_{d}\right)\end{array}$ & Reference \\
\hline \multirow[t]{3}{*}{ Aflatoxin $\mathrm{B}_{1}$} & $\begin{array}{l}\mathrm{AFB}_{1} \\
\text { Aptamer }\end{array}$ & $\begin{array}{l}\text { GTTGGGCACGTGTTGTCTCTCTGTGTCTC } \\
\text { GTGCCCTTCGCTAGGCCCACA }\end{array}$ & $\sim 2.00 \mu \mathrm{M}$ & 70 \\
\hline & $\begin{array}{l}\mathrm{AFB}_{1} \\
\text { Seq. } 1\end{array}$ & $\begin{array}{l}\text { AGCAGCACAGAGGTCAGATGGTGCTAT } \\
\text { CATGCGCTCAATGGGAGA } \\
\text { CTTTAGCTGCCCCCACCTATGCGTGCTA } \\
\text { CCGTGAA }\end{array}$ & $\begin{array}{l}11.29 \pm 1.27 \mathrm{nM} \\
\text { (fluorescent } \\
\text { bioassay) }\end{array}$ & 67 \\
\hline & AFAB3 & $\begin{array}{l}\text { ATCCGTCACACCTGCTCTATTCCTCTGTT } \\
\text { GAAGAACCACTTCCGGAAATAAGAGTG } \\
\text { GTGTTGGCTCCCGTAT }\end{array}$ & $\begin{array}{l}96.0 \pm 8.6 \mathrm{nM} \\
\text { (magnetic bead } \\
\text { assay) }\end{array}$ & 68 \\
\hline Aflatoxin $\mathrm{B}_{2}$ & $\begin{array}{l}\mathrm{AFB}_{1} \\
\text { Seq. } 17\end{array}$ & $\begin{array}{l}\text { AGCAGCACAGAGGTCAGATGCTGACAC } \\
\text { CCTGGACCTTGGGATTCCGGAAGTTTTC } \\
\text { CGGTACCTATGCGTGCTACCGTGAA }\end{array}$ & $\begin{array}{l}9.83 \pm 0.99 \mathrm{nM} \\
\text { (fluorescent } \\
\text { bioassay) }\end{array}$ & 69 \\
\hline \multirow[t]{2}{*}{ Aflatoxin $\mathrm{M}_{1}$} & AFAS3 & $\begin{array}{l}\text { ATCCGTCACACCTGCTCTGACGCTGGGG } \\
\text { TCGACCCGGAGAAATGCATTCCCCTGTG } \\
\text { GTGTTGGCTCCCGTAT }\end{array}$ & $\begin{array}{c}35.6 \pm 2.6 \mathrm{nM} \\
\text { (magnetic bead } \\
\text { assay) }\end{array}$ & 68 \\
\hline & $\begin{array}{l}\mathrm{AFM}_{1} \\
\text { Aptamer }\end{array}$ & ACTGCTAGAGATTTTCCACAT & - & 71 \\
\hline
\end{tabular}

\subsection{Aptasensors}

In their simplest form, biosensors are analytical devices that use a biological or biologically derived component to transduce a binding event into a measurable signal. ${ }^{72}$ Biosensors that incorporate aptamers as recognition elements are commonly referred to as aptasensors. Aptamers have been incorporated into a variety of pre-existing antibodybased sensing systems. Based on certain inherent advantages that aptamers offer, such as their low production cost, and ease of modification, the development of novel sensing platforms has led aptamers to become unique in their use as molecular recognition elements. ${ }^{73}$ Sensing platforms that are unique to aptamers often benefit from the direct 
readout of ligand binding. ${ }^{74}$ Aptamers are readily modified in a site-specific manner and can be developed to undergo conformational changes upon complex formation. Additionally, they can be synthesized readily. Possible signal transduction methods include electrical, electrochemical, optical, piezoelectric, and mass-based. ${ }^{73,75}$

Mass-based signal transduction such as surface plasmon resonance (SPR) has been widely used. They are commonly referred to as label free techniques. In short, the change in conductive properties of a metal surface is altered when the target is bound to a surface immobilized aptamer. The produced signals are proportional to the differential changes in mass. ${ }^{73,76}$ Electrochemical sensors, as well, have produced very sensitive sensors for aflatoxins. Aptamer functionalized gold coated glassy carbon electrodes have been utilized for electrical impedance spectroscopy. Other electrochemical sensors have utilized enzyme-catalyzed target recycling for signal amplification. ${ }^{77,78}$ There are also many approaches to optical sensing. Commonly used colorimetric assays utilize spherical gold nanoparticles (AuNPs) for their unique optical properties. ${ }^{79}$ Fluorescence-based biosensing is one of the most popular, and sensitive methods for the design of optical aptasensors. Further signal transduction schemes for fluorescent aptasensors will be described in Section 1.3.1.

\subsubsection{Optical aptasensors}

Many optical aptasensing techniques exploit the conformational changes that occur in an aptamer upon ligand binding. Many different signal transduction schemes have been reported. As previously stated, fluorescence detection is fast becoming one of the most popular methods for signal transduction in aptasensors. Many conventional 
diagnostic tools utilize immobilized recognition elements or target immobilization. These techniques are often laborious and costly. The development of homogeneous fluorescent assays, on the other hand, allows the elimination of many sample preparation steps, reduce the effects of non-specific interactions, and can potentially reduce cost. ${ }^{72,80}$ Standard DNA molecules do not exhibit intrinsic fluorescence, therefore, the fluorescence signal is not produced by the aptamer directly. The introduction of covalently linked organic dyes in predetermined sequence regions is a valuable tool for the design of aptamer-based sensors. The use of organic chromophores, as well as nanoparticle reporters, means that spectrofluorometric sensing techniques are not limited to detection of ligands with intrinsic fluorescence. Also, given the wide variety of commercially available dyes, a vast array sensor designs are possible. 
Table 1.4: Summary of recent aptasensors developed for aflatoxin contaminants (2014-2017), ordered by the target, then year. (Table adapted from Ref. ${ }^{81}$ )

\begin{tabular}{|c|c|c|c|}
\hline Target & Aptasensor Description & LOD [LDR] & Reference \\
\hline \multirow[t]{20}{*}{ Aflatoxin $\mathrm{B}_{1}$} & $\begin{array}{l}\text { Electrochemical ( } \pi \text {-shaped Apt double layer on } \\
\text { Au electrode) }\end{array}$ & $\begin{array}{l}2 \mathrm{pg} / \mathrm{mL} \\
{[7-500 \mathrm{pg} / \mathrm{mL}]}\end{array}$ & 78 \\
\hline & $\begin{array}{l}\text { Fluorometric (FRET - competitive } \\
\text { displacement of FAM and TAMRA mod. } \\
\text { probes) }\end{array}$ & $\begin{array}{l}1.6 \mathrm{ng} / \mathrm{mL} \\
{[5-100 \mathrm{ng} / \mathrm{mL}]}\end{array}$ & 82 \\
\hline & $\begin{array}{l}\text { Fluorometric (FRET - FAM labelled aptamer } \\
\text { quenching by GO) }\end{array}$ & $4.5 \mathrm{ppb}$ & 83 \\
\hline & Aptamer coated SPR chip & $\begin{array}{l}0.4 \mathrm{nM} \\
{[0.4-200 \mathrm{nM}]}\end{array}$ & 76 \\
\hline & $\begin{array}{l}\text { Fluorometric (FRET - competitive } \\
\text { displacement of FAM and TAMRA mod. DNA }\end{array}$ & $\begin{array}{l}0.2 \mathrm{ng} / \mathrm{mL} \\
{[0.25-32 \mathrm{ng} / \mathrm{mL}]}\end{array}$ & 84 \\
\hline & $\begin{array}{l}\text { Fluorometric (RT-qPCR with aptamer } \\
\text { recognition element) }\end{array}$ & $\begin{array}{l}0.03 \mathrm{ng} / \mathrm{L} \\
{[0.0001-1 \mu \mathrm{g} / \mathrm{L}]}\end{array}$ & 85 \\
\hline & $\begin{array}{l}\text { Protein-encoded aptamer nanomachine for } \\
\text { fluorescence polarization (FP) }\end{array}$ & $0.24 \mathrm{ppm}$ & 86 \\
\hline & Colourimetric ( $\mathrm{AFB}_{1}$ responsive hydrogel) & $0.55 \mathrm{ppb}$ & 79 \\
\hline & $\begin{array}{l}\text { Fluorometric (N-doped carbon dots on aptamer } \\
\text { modified AuNPs) }\end{array}$ & $\begin{array}{l}5 \mathrm{pg} / \mathrm{mL} \\
{[5-200 \mathrm{pg} / \mathrm{mL}]}\end{array}$ & 87 \\
\hline & $\begin{array}{l}\text { Electrochemical (aptamer conjugated SPCEs for } \\
\text { EIS) }\end{array}$ & $\begin{array}{l}0.12 \mathrm{ng} / \mathrm{mL} \\
{[0.125-16 \mathrm{ng} / \mathrm{mL}]}\end{array}$ & 88 \\
\hline & $\begin{array}{l}\text { Fluorometric (DNA-scaffolded silver } \\
\text { nanoclusters) }\end{array}$ & $\begin{array}{l}0.3 \mathrm{pg} / \mathrm{mL} \\
{[0.001-0.05 \mathrm{ng} / \mathrm{mL}]}\end{array}$ & 89 \\
\hline & $\begin{array}{l}\text { Electrochemical (EXO III two-round signal } \\
\text { amplification) }\end{array}$ & $0.0006 \mathrm{ppt}$ & 77 \\
\hline & $\begin{array}{l}\text { Electrochemical (redox indicator utilized in } \\
\text { multi-layer framework for CV and EIS) }\end{array}$ & $\begin{array}{l}0.40 \mathrm{nM} \\
{[0.1-10 \mathrm{nM}]}\end{array}$ & 90 \\
\hline & $\begin{array}{l}\text { Electrochemical (electropolymerization of } \\
\text { functionalized AuNPs in presence of } \mathrm{AFB}_{1} \text { ) }\end{array}$ & $\begin{array}{l}3.0 \mathrm{fM} \\
{[3.2 \mathrm{fM}-3.2 \mu \mathrm{M}]}\end{array}$ & 91 \\
\hline & $\begin{array}{l}\text { Fluorometric (FRET - quenching of aptamer } \\
\text { mod. Q-dots by GO) }\end{array}$ & $\begin{array}{l}1.4 \mathrm{nM} \\
{[1.6 \mathrm{nM}-160 \mu \mathrm{M}]}\end{array}$ & 92 \\
\hline & $\begin{array}{l}\text { Colourimetric (split DNAzyme probes with } \\
\text { HRP mimicking activity) }\end{array}$ & $\begin{array}{l}0.1 \mathrm{ng} / \mathrm{mL} \\
{\left[0.1-10^{\prime} 000 \mathrm{ng} / \mathrm{mL}\right]}\end{array}$ & 93 \\
\hline & $\begin{array}{l}\text { SERS using Ag@Au core-shell nanoparticle } \\
\text { tags }\end{array}$ & $\begin{array}{l}0.03 \mathrm{ng} / \mathrm{mL} \\
{[0.05-100 \mathrm{ng} / \mathrm{mL}]}\end{array}$ & 94 \\
\hline & $\begin{array}{l}\text { Fluorometric (signal amplification by RT- } \\
\text { qPCR) }\end{array}$ & $\begin{array}{l}25 \mathrm{fg} / \mathrm{mL} \\
{\left[5.0 \times 10^{-5}-5 \mathrm{ng} / \mathrm{mL}\right]}\end{array}$ & 95 \\
\hline & Fluorometric (aptamer-based dipstick assay) & $0.1 \mathrm{ng} / \mathrm{mL}$ & 96 \\
\hline & $\begin{array}{l}\text { Chemiluminescent (HRP-mimicking } \\
\text { competitive aptamer assay) }\end{array}$ & $\begin{array}{l}0.11 \mathrm{ng} / \mathrm{mL} \\
{[0.1-10 \mathrm{ng} / \mathrm{mL}]}\end{array}$ & 97 \\
\hline Aflatoxin $B_{2}$ & $\begin{array}{l}\text { Fluorometric (magnetic separation of FAM } \\
\text { labelled aptamer) }\end{array}$ & $\begin{array}{l}50 \mathrm{ng} / \mathrm{L} \\
{[100-1800 \mathrm{ng} / \mathrm{L}]}\end{array}$ & 69 \\
\hline \multirow[t]{2}{*}{ Aflatoxin M1 } & $\begin{array}{l}\text { ECL-RET - Between luminol donor and } \\
\mathrm{Ru}(\mathrm{bpy}){ }_{3}{ }^{2+} \text { acceptor }\end{array}$ & $0.02 \mathrm{pg} / \mathrm{mL}$ & 98 \\
\hline & Electrochemical (aptamer recognition in EIS) & $\begin{array}{l}1.15 \mathrm{ng} / \mathrm{L} \\
{[2-150 \mathrm{ng} / \mathrm{L}]}\end{array}$ & 99 \\
\hline
\end{tabular}


Focusing on the development of aflatoxin sensing schemes, a simple aptamerbased fluorescent sensor similar to the designs proposed in this text was reported by Chen et al. The strategy utilized two fluorophore labelled complementary sequences. The duplex formation allows the quenching of fluorescein by TAMRA. In the presence of AFB1, the release of the complementary strand creates a spatial separation of the two chromophores and a recovery in fluorescence. ${ }^{82}$ Another alternative to fluorescent sensing scheme that has become more prominent is the use the fluorescence quenching ability of graphene oxide (GO). Joo et al. (2017) reported the development of a "signal off" Terminally labelled FAM-aptamer was used for its structure dependent interactions with GO. Ligand-binding induced conformational changes resulted in the adsorption of aptamer/target complex onto GO resulting in a decrease in fluorescence. The sensing platform achieved a limit of detection of $4.5 \mathrm{ppb} .^{92}$

Fluorescent sensors are not limited to using commercially available organic dyes. Slightly more complex transduction strategies can be used with the incorporation of nanoparticle reporters. AuNPs and QDs along with other nanoparticles offer a number of advantages over organic dyes. Due to their unique optical properties, nanoparticle reporters tend to exhibit greater photostability and, longer fluorescent lifetimes, and sharper emission bands. A novel fluorescent sensor was reported in 2016 by Wang et al. The strategy utilized nitrogen-doped carbon dots assembled onto aptamer modified AuNPs. In the presence of $\mathrm{AFB}_{1}$, the carbon dots were released from the surface of the AuNPs causing a recovery of fluorescence intensity. The method exhibited extremely high sensitivity with a detection limit of $5 \mathrm{pg} / \mathrm{mL} .{ }^{87}$ Interestingly, a number of simple homogeneous sensing platforms, that minimize the number of components in a sensor, 
have reported detection limits that rival those of more complex signal transduction schemes. While in some cases, these techniques require a more in-depth understanding of aptamer/target interactions that are occurring, they provide the added benefits of being cost-effective, and simplistic in both use and production. Moreover, the end goal of many biosensor applications for mycotoxin contamination is their use as on-site detection tools. High mycotoxin exposure occurs primarily in developing countries. In order for theses sensing platforms to be marketable, the benefits highlighted above should be of primary concern.

\section{$1.4 \quad$ Thesis objectives}

The characterization and application of aflatoxin aptamer candidates which were previously selected through a specialized selection method known as capture-SELEX, are the focus of this work. The aptamer candidates for aflatoxin will undergo structural characterization using an adapted electrophoretic mobility shift assay (EMSA) technique, melting temperature, circular dichroism, and predictive software tools. Aptamer binding affinity will also be determined for the four major aflatoxin targets $\left(\mathrm{AFB}_{1}, \mathrm{AFB}_{2}, \mathrm{AFG}_{1}\right.$, and $\mathrm{AFG}_{2}$ ). From this, top aptamer candidates will be used in the development of a FRET-based hybridized signalling aptamer sensing platform that can be utilized as a sensitive, selective, and cost effective pre-screening method for aflatoxin analysis in food samples. 


\section{Chapter: Aptamer Characterization}

\subsection{Statement of contribution}

The aptamer candidate selection and screening EMSA experiments were performed by Erin McConnell. Preliminary specificity tests with EMSA were performed by Erin McConnell and Daniel Goudreau. All other experiments and synthesis were performed by Daniel Goudreau.

\subsection{Introduction}

\subsubsection{Aptamer selection}

The aptamer candidates in question were previously selected by the DeRosa group through a multi-pool capture SELEX. Aptamers contained a fixed central domain that was flanked by two randomized regions and primer binding regions. The SELEX protocol was adapted from work done by Nutiu and $\mathrm{Li}$ (2005) on structure switching signalling aptamers. Nutiu and Li reported the selection of unmodified duplexed aptamers that could be applied effectively as signalling probes with little need for optimization. ${ }^{57}$ The DNA library was immobilized onto a solid support through a 12-base complementary region incorporated into the fixed central domain. An antisense, sequence 24-nt in length, containing a 5 ' biotinylated poly T-tail (12-nt), referred to as the capture probe $(\mathrm{CP})$, allowed the DNA library to be immobilized onto streptavidin functionalized agarose beads. 


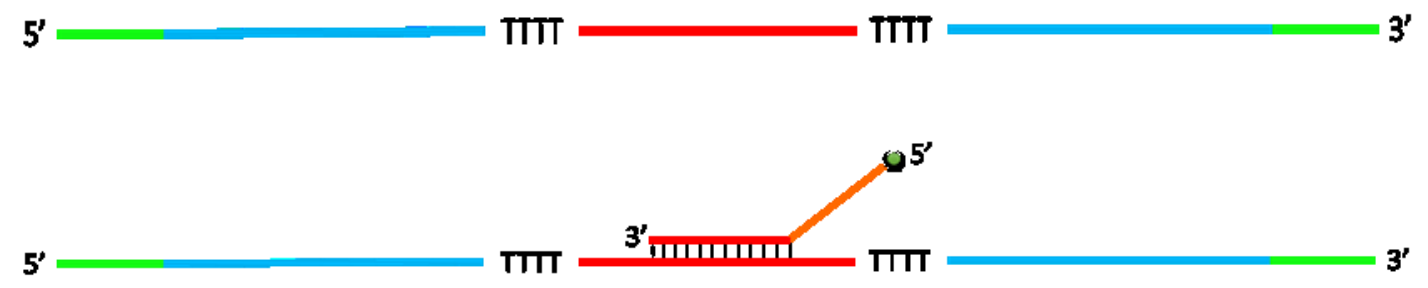

Figure 3.1: Schematic representation of sequence template with fixed internal sequence (Red), flanking randomized regions (Blue), primer binding regions (Green), and biotinylated capture probe.

Table 3.1: Sequence library templates for DZA and MR libraries and their respective complementary elements used for immobilization.

\begin{tabular}{ll}
\hline DNA Template & Sequence (5' to 3') \\
\hline DZA & AGCAGCACAGAGGTCAGATG-N15- \\
& TTTTGTGGGTAGGGCGGGTTGGTTTT-N15- \\
& CCTATGCGTGCTACCGTGAA \\
\hline $3 \mathrm{MR}$ & AGCAGCACAGAGGTCAGATG-N25- \\
& GACTTCGTTCGAGGCTCAT-N25- \\
& CCTATGCGTGCTACCGTGAA \\
\hline DZ CP & Bio - TTTTTTTTTTTTAACCCGCCCTAC \\
\hline MR CP & Bio - TTTTTTTTTTTTECCTCGAACGAA \\
\hline
\end{tabular}

The first template, referred to as DZA, was 96 nucleotides (nt) in length and contained 15-nt random regions. The template was designed to contain a known internal sequence that favoured the formation of a G-quadruplex. The biased sequence domain, PS2.M (5'GTGGGTAGGGCGGGTTGG-3'), is a short 18-nt parallel G-quadruplex forming sequence previously reported by Travascio et al. (1998). It was reported to fold unimolecularly with two single-base loos and two double-base loops. ${ }^{100}$ The porphyrinbinding sequence was originally selected to form a pseudo-catalytic DNA-hemin complex that could rival hemin-antibody complexes. The sequence was shown to bind hemin (Fe(III)-protoporphyrinIX) with submicromolar affinity. The DNA-Hemin complex formed via the intercalation of hemin in the G-quadruplexes exhibited DNA- 
enhanced peroxidative activity. ${ }^{101}$ In comparison to fully randomized sequences, which are expected to have near $25 \%$ guanine, the DZA aptamer candidates have a high occurrence of guanine residues due to their fixed guanine rich domain. The guanine contents of the DZA candidates range from $30.2 \%$ to $34.4 \%$, with DZA7 being the highest (see Table 3.2 and Table 3.3).

The second template, 3MR, was 100-nt in length, containing a 19-nt central sequence, and 25-nt random regions. The MR template contained a fixed capture region, common to all sequences, however, they were not designed to contain any structural biases. In total, 5 selection rounds were performed. Positive elutions were performed using aflatoxins $B_{1}, B_{2}, G_{1}, G_{2}$ in a 5:1:1:1 ratio to select for a total aflatoxin aptamer. And the final round was performed using 3M SELEX buffer containing 5\% BSA and corn extract.

Table 3.2: Full-length candidate sequences from the DZA templated library

\begin{tabular}{|c|c|c|c|}
\hline Name & Sequence (5' to 3') & $\begin{array}{c}\text { Length } \\
\text { (nt) }\end{array}$ & $\begin{array}{c}\text { G } \\
\text { content } \\
(\%)\end{array}$ \\
\hline DZA3 & $\begin{array}{l}\text { AGCAGCACAGAGGTCAGATGGCATGACAAGTAAA } \\
\text { GTTTTGTGGGTAGGGCGGGTTGGTTTTGTTGACAG } \\
\text { ATGTATCCCTATGCGTGCTACCGTGAA }\end{array}$ & 96 & 33.3 \\
\hline DZA5 & $\begin{array}{l}\text { AGCAGCACAGAGGTCAGATGGATACATCTGTCAAC } \\
\text { TTTTGTGGGTAGGGCGGGTTGGTTTTGACATCTGAC } \\
\text { CTCTGCCTATGCGTGCTACCGTGAA }\end{array}$ & 96 & 30.2 \\
\hline DZA7 & $\begin{array}{l}\text { AGCAGCACAGAGGTCAGATGGATACATCTGTCAAC } \\
\text { TTTTGTGGGTAGGGCGGGTTGGTAGAGTGTAGATCT } \\
\text { CGGTGCCTATGCGTGCTACCGTGAA }\end{array}$ & 96 & 34.4 \\
\hline
\end{tabular}


Table 3.3: Full-length candidate sequences from the random aptamer templated library

\begin{tabular}{clcc}
\hline Name & Sequence (5' to 3') & $\begin{array}{c}\text { Length } \\
\text { (nt) }\end{array}$ & $\begin{array}{c}\text { G } \\
\text { content } \\
\text { (\%) }\end{array}$ \\
\hline 3MR2 & $\begin{array}{l}\text { GAGGTCAGATGCAATCCAAACTTTGTTACTCGTCAGA } \\
\text { AATTCGTTCGAGGCTTCCACGGCGCTTTAAAATAGTTG } \\
\text { TTATACCTATGCGTGCTACCGTGAA }\end{array}$ & 100 & 22.0 \\
\hline 3MR3 & $\begin{array}{l}\text { GAGGTCAGATGGGTCTGTTGAACACGACCAGAAAAC } \\
\text { TGGTTCGTTCGAGGCTTCCACGGCGCTTTAAAATAGTT } \\
\text { GTTATACCTATGCGTGCTACCGTGAA }\end{array}$ & 100 & 27.0 \\
\hline 3MR5 & $\begin{array}{l}\text { CAGCACAGAGGTCAGATGTGGCGCCTCACACGTGCA } \\
\text { ATCGAATGACTTCGTTCGAGGCTCATAATCGTCCTCGG } \\
\text { TCTATGATGTATCCCTATGCGTGCTA }\end{array}$ & 100 & 25.0 \\
\hline
\end{tabular}

\subsubsection{Structural characterization of nucleic acid aptamers}

\subsubsection{G-quadruplex structures}

Hydrogen bonding plays a key role in the structural stabilisation of macromolecules. G-quadruplexes are tetra helical nucleic acid structures that are formed by tandem repeats of short guanine rich sequences. G-quartet motifs are formed via Hoogsteen hydrogen bonding between four guanines. ${ }^{102,103}$ Quadruplexes are formed of two or more stacked planes that are stabilized by $\pi-\pi$ interaction. Conformational arrangements of G-quadruplex structures are largely monovalent cation-dependent. The strong negative electrostatic potential created by the guanine O6 oxygens that form the central channel of the G-quadruplex. Both monovalent and small divalent cation bound forms can adopt the same folding topology, where 16-guanine bases build a central core of four stacked hydrogen-bonded quartets. ${ }^{103,104}$ Quadruplexes require monovalent or small divalent cations for the preservation of their complex structures. Monovalent cation positions alter the loop conformation. $\mathrm{K}^{+}$ions typically intercalate between the planes of 
the quartets. Smaller $\mathrm{Na}^{+}$ions are arranged in a coplanar fashion within the quartets. Furthermore, they are less sterically constrained and can adopt a range of positions that reduce electrostatic repulsion. ${ }^{105}$ G-quadruplexes can be formed by the repeated folding of a single strand (intramolecular), or by interactions between multiple strands, typically two and four. ${ }^{106} \mathrm{G}$-quadruplex-forming repeat sequences have been determined in telomeric DNA. Telomeric G-quadruplexes have been shown to suppress and reduce telomerase activity. Non-telomeric DNA plays a role in regulating RNA transcription based on their proximity to promoter regions. ${ }^{107}$ Figure 3.2 shows common G-quadruplex topologies. Sodium stabilized quadruplexes typically exhibit anti-parallel strand orientation in a basket-type structure as illustrated in Figure 3.2C). Potassium stabilized quadruplexes can adopt topologies a variety of topologies including parallel, and antiparallel chair conformations as illustrated in Figure 3.2A) and B).

Several small organic compounds have been known to intercalate and selectively promote the formation G-quadruplexes. Examples of these include anthraquinone, porphyrins, and acridines. ${ }^{108}$ The most well-known use of G-quadruplexes in synthetic oligonucleotides is in the thrombin binding aptamer. The predictable intramolecular folding of the G-quadruplex containing aptamer causes 5' and 3' ends to be in closer proximity to each other when thrombin is intercalated in the G-quadruplex making it ideal for monitoring FRET. ${ }^{109}$ 

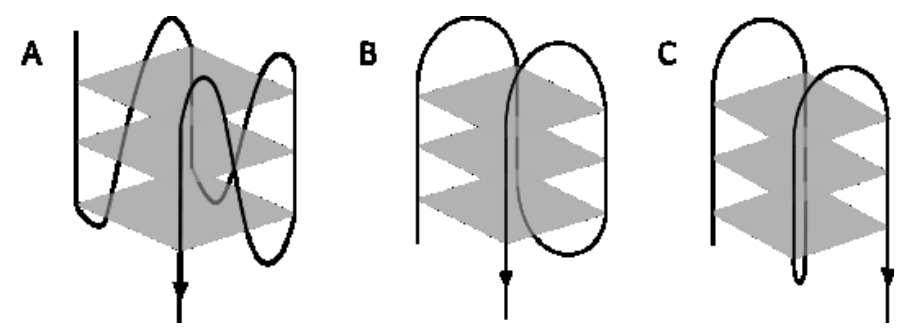

Figure 3.2: Graphical representation of common G-quadruplex topologies A) parallel G-quadruplex with anti conformation of the guanine glycosyl bonds B) antiparallel G-quadruplex adopting the chair conformation $C$ ) antiparallel G-quadruplex structure containing a diagonal loop. Both $B$ \&C contain anti/syn alternating conformation of the glycosyl bonds.

\subsubsection{Predictive analysis: G-quadruplex mapping}

There are a number of available computational programs developed for the predictive analysis of DNA structure. RNA structure and UNAFold are examples of programs that are used to predict the probability of base pair interaction based on thermodynamic parameters. The tools are useful in terms of preliminary structure characterization when analysed comparatively. The resulting structure predictions are limited to two-dimensional conformations and cannot predict complex structures such as G-quadruplexes or structures associated with DNA-ligand interactions.

Other prediction software uses the sequence order to predict the location and the probability of higher order structure formation. The study of G-quadruplex forming sequences has gained traction due to the supporting evidence that quadruplex structures are involved in biological processes such as DNA replication and cell proliferation. Gquadruplexes have been identified near promoter regions and other biologically significant regions of the mammalian genome. ${ }^{106}$ Kikin et al. describe the development of an easily accessible web-based server that can be used to study the ability of an oligonucleotide sequence to form G-quadruplexes and their distribution in guanine rich 
sequences. Moreover, the tool allows users to search all NCBI databases for gene/nucleotide sequences. ${ }^{106}$ The quadruplex forming guanine rich sequences (QGRS) mapper predicts the location of motifs using the following arrangement:

\section{$G_{x} N_{y 1} G_{x} N_{y 2} G_{x} N_{y 3} G_{x}$}

Where $\mathrm{x}$ is the number of guanine tetrads in the quadruplex $\mathrm{y}$ is the length of the loops connecting the tetrads.

A scoring system called G-score uses the above configuration to predict the formation of a stable G-quadruplex. Based on previous literature, shorter and similar loop sizes, as well as a higher number of guanine tetrads $(x \geq 2)$ favour the more stable quadruplexes..$^{110,111}$ The QGRS mapper can be a useful tool in the rational design of aptamers that have a high propensity for forming G-quadruplexes. The current program is suited to predicting the formation of intramolecular quadruplex. Future advances could potentially allow the prediction of intermolecular quadruplexes. This could be a useful tool in the design of label free sensing platforms.

\subsubsection{Circular Dichroism}

Circular dichroism (CD) spectroscopy originated from the study of chiral molecules with circularly polarized light (CPL). Due to their chiral nature, $\mathrm{CD}$ has become a very useful technique for the study of biomolecules, particularly proteins and nucleic acids. CD spectrophotometers record the differential absorption of polarized light along a range of wavelengths. Since $\mathrm{CD}$ depends on the conformation of the oligonucleotide and not on the sum of its individual base constituents, ultra-violet CD has 
become an appropriate tool for studying folding pathways of G-quadruplexes and their varied topologies among other conformational features.

Of the many G-quadruplex topologies, there are two principle topological groups, the first being parallel and the second being the antiparallel quadruplex. Within the UV region of interest $(220-350 \mathrm{~nm})$, there are 4 distinct bands that are commonly observed. These bands are typically centred at $\sim 245, \sim 255, \sim 275$, and $\sim 295 \mathrm{~nm} .{ }^{112}$ The parallel Gquadruplex is described as a right-handed helix with fourfold symmetry in which all guanines have anti conformation of the glycosyl bonds. CD spectra belonging to parallel quadruplexes tend to show a maximum in ellipticity near $260 \mathrm{~nm}$, and a smaller negative band near $240 \mathrm{~nm} \cdot{ }^{113}$ anti-parallel conformations differ in their stacking interactions. Guanines have alternating syn and anti conformations of the glycosyl bonds (see Figure 3.3). The CD spectra of an antiparallel quadruplex exhibits a positive band near $295 \mathrm{~nm}$, a negative band shifted to $260 \mathrm{~nm}$, and a third positive band at $245 \mathrm{~nm} \cdot{ }^{37,113}$ Since CD is very sensitive to base-stacking geometries, it is suggested that the polarity of stacked quartets is the factor that affects spectral signatures and not the relative orientation of the sugar-phosphate backbone. ${ }^{113}$ 

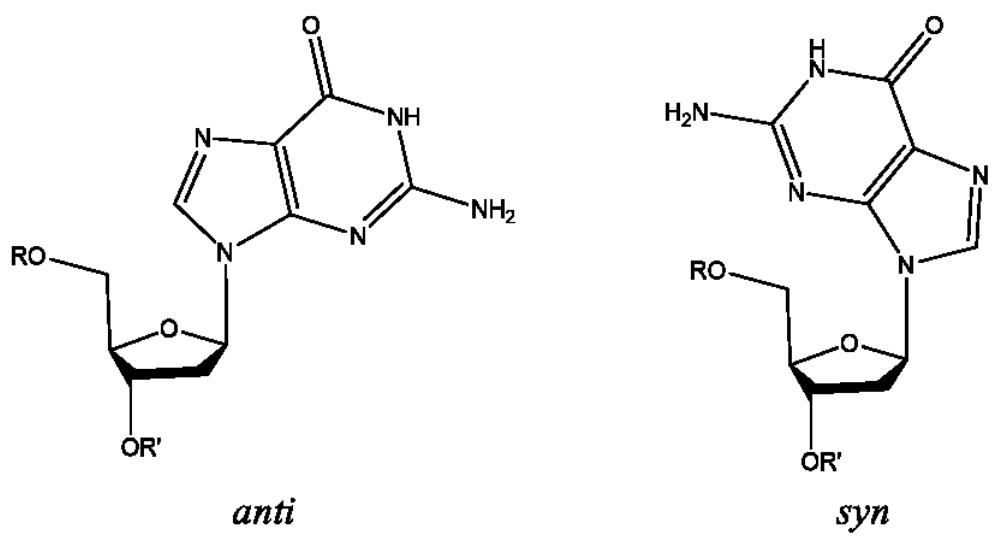

Figure 3.3: Chemical structures representing the anti and syn glycosidic bond angles.

Due to the complexity of large DNA molecules, structural analysis by CD spectroscopy is grossly empirical. The comparison of G-quadruplexes based on the CD profiles of typified parallel and anti-parallel telomeric quadruplexes has proven to be reliable in simple quadruplex forming sequences. However, complex DNA sequences are not as easily elucidated. ${ }^{104,113}$

\subsubsection{Measuring dissociation constants by microscale thermophoresis}

Microscale thermophoresis (MST) is a useful and precise fluorescence-based technique used to quantify biomolecular interactions including DNA hybridization, protein-DNA interactions, and protein-protein interactions. MST is based on the directed movement of molecules along a temperature gradient, often called thermophoresis or thermodiffusion. This diffusion depends on properties such as charge, conformation, hydration shell and size. Small molecule interactions can be detected due to changes in charge and hydration shell. Typically, samples are loaded into thin glass capillaries and temperature gradients are established by an IR laser. Changes in fluorescence are 
monitored as DNA depletion occurs along the temperature gradient and convection drives DNA stagnation against the cold capillary surface. ${ }^{114,115}$ MST can be performed using the intrinsic fluorescent properties of a molecule. Alternatively, aptamers can be fluorescently labelled with little restrictions since they are free in solution. ${ }^{65}$

$\mathrm{K}_{\mathrm{d}}$ from law of mass action. ${ }^{114}$

$$
\begin{gathered}
A+T \leftrightarrow A T \\
F\left(C_{T}\right)=F_{u}+\left(F_{b}-F_{u}\right) \times \frac{C_{A T}}{C_{A}}, \\
\frac{C_{A T}}{C_{A}}=\text { Fraction bound } \\
=\frac{1}{2 C_{A}} \times\left(C_{T}+C_{A}+K_{d}-\sqrt{\left(\left(C_{T}+C_{A}+K_{d}\right)^{2}-4 C_{T} C_{A}\right)}\right.
\end{gathered}
$$

Where, $\mathrm{F}_{\mathrm{u}}$ fluorescence in unbound state

$\mathrm{F}_{\mathrm{b}}$ fluorescence in the bound state.

$\mathrm{K}_{\mathrm{d}}$ dissociation constant, to be determined.

$\mathrm{C}_{\mathrm{AT}}$ concentration of formed complex.

$\mathrm{C}_{\mathrm{A}}$ constant concentration of molecule A known.

$\mathrm{C}_{\mathrm{T}}$ provided concentration of titrated molecule $\mathrm{T}$.

Basic binding parameters are crucial for understanding aptamer-small molecule interactions for impending applications. MST provides several advantages over other methods that rely on biomolecule surface immobilization. For instance, immobilization of biomolecules and surface dependent changes in binding kinetics that appear in surface plasmon resonance techniques are avoided. MST is also capable of detection at low $\mathrm{pM}$ 
concentrations. It has low sample consumption $(\sim 6 \mu \mathrm{L})$ and offers free choice of buffers including complex matrices.

\subsubsection{Chapter objectives}

This chapter describes the use of multiple techniques for the screening and conformational analysis of two structurally different aptamer candidate groups selected for total aflatoxins $\left(\mathrm{AFB}_{1}, \mathrm{AFB}_{2}, \mathrm{AFG}_{1}, \mathrm{AFG}_{2}\right.$ mixture). Aptamer candidates will undergo further characterization using a solution-based binding affinity assay. Optimal candidates will find further use as molecular recognition elements for the development of inexpensive, robust and innovative fluorescent biosensor platforms.

\subsection{Experimental}

\subsubsection{Materials}

DZA and 3MR Oligonucleotide sequences were purchased from Integrated DNA Technologies (Coralville, Iowa, USA). Additional DZA3 aptamer was synthesized via solution-phase phosphoramidite, oligo synthesis method on a BioAutomation Mermade 6 oligonucleotide synthesizer. Phosphoramidites, sequence modifiers, activator, deblock, capping agents, and oxidizing agents were purchased from Glen Research. HPLC grade Acetonitrile was purchased from EMD Millipore. Controlled pore glass (CPG) support columns were purchased from BioAutomation. All buffers and solutions were prepared with EMD Millipore, Milli-Q deionized water $\left(18.2 \mathrm{M} \Omega \cdot \mathrm{cm} @ 25^{\circ} \mathrm{C}\right)$. Buffering agents and salts were purchased from Sigma-Aldrich. Ultra-high purity 5.0 argon was supplied by Praxair. Aflatoxin $\mathrm{B}_{1}$ and $\mathrm{G}_{1}$ from Aspergillus flavus $\geq 98.00 \%$ (HPLC), and 
Aflatoxins $\mathrm{B}_{2}$ and $\mathrm{G}_{2} \geq 98.00 \%$ (TLC) were purchased from Sigma-Aldrich.

Acrylamide/bis-acrylamide, $40 \%$ solution, electrophoresis grade ammonium persulfate, $\mathrm{N}, \mathrm{N}, \mathrm{N}$ ', N'-tetramethylethylenediamine (TEMED), ethylenediaminetetraacetic acid (EDTA), Boric acid, tris(hydroxymethyl) aminomethane (Tris) and urea were all purchased from Bioshop Canada Inc. Amicon-Ultra $0.5 \mathrm{~mL} 3 \mathrm{kDa}$ centrifugal filters were purchased from Sigma-Aldrich. PAGE gels were imaged using an Alpha Innotech multiimage light cabinet. UV/Visible absorption spectra were acquired using a Varian Cary 300 Bio UV/Visible spectrophotometer.

\subsubsection{Buffers}

Table 3.4: Composition of buffers used in chapter 2

\begin{tabular}{ccc}
\hline Buffer & Composition & pH \\
\hline $3 \mathrm{M} \mathrm{Selex} \mathrm{Buffer}$ & $20 \mathrm{mM}$ Tris, $100 \mathrm{mM} \mathrm{NaCl}, 5 \mathrm{mM} \mathrm{KCl}, 1 \mathrm{mM} \mathrm{CaCl}_{2}$ & 7.4 \\
\hline $3 \mathrm{M} \mathrm{Mg}$ Buffer & $20 \mathrm{mM}$ Tris, $100 \mathrm{mM} \mathrm{NaCl}, 5 \mathrm{mM} \mathrm{KCl}, 1 \mathrm{mM} \mathrm{CaCl}_{2}, 1$ & 7.4 \\
& $\mathrm{mM} \mathrm{MgCl}_{2}$ & \\
\hline
\end{tabular}

\subsubsection{DNA synthesis, purification, and quantification}

Oligonucleotide sequence DZA3 was synthesized using standard phosphoramidite chemistry. $0.067 \mathrm{M}$ solutions of guanosine, thymidine, adenosine, and cytidine phosphoramidites were prepared by dissolving $100 \mu \mathrm{mol}$ samples in $1.5 \mathrm{~mL}$ of HPLC grade acetonitrile (Glen Research). 0.067 M Black Hole Quencher 1 (BHQ-1-dT) modifier was prepared with a 1:3(v/v), anhydrous acetonitrile/dichloromethane diluent, and $0.067 \mathrm{M}$ fluorescein-dT phosphoramidite solution was prepared with anhydrous acetonitrile. Phosphoramidite solutions and $1000 \AA$ CPG support columns were loaded onto a BioAutomation Mermade 6 synthesizer. Sequences were synthesized following 
manufacturer suggested conditions. Coupling time was increased to 10 minutes for all modifiers (BHQ-1-dT, and Fluorescein-dT).

Following synthesis, support columns were removed from the synthesizer. Each columns' CPG support beads were carefully transferred to separate microcentrifuge tubes, and allowed to incubate in $1 \mathrm{~mL}$ of $28 \% \mathrm{NH}_{4} \mathrm{OH}$ for $17-24$ hours at $55^{\circ} \mathrm{C}$. Following the cleavage and deprotection steps, the tubes were centrifuged, and the supernatant was transferred to a series of clean microcentrifuge tubes. The beads were washed with an additional $1 \mathrm{~mL}$ of deionized water. Both $\mathrm{NH}_{4} \mathrm{OH}$ and water fractions were collected and dried overnight on a Savant AES2010 SpeedVac cryopump.

\subsubsection{PAGE purification}

The oligonucleotides were then purified using $12 \%$ denaturing polyacrylamide gel electrophoresis (PAGE). Gel solutions were prepared by mixing $63 \mathrm{~g}$ of urea, $47 \mathrm{~mL}$ of acrylamide solution (acrylamide/bisacrylamide, 40\% solution, 19:1), $30 \mathrm{~mL}$ of 5X TBE buffer (45 mM Tris, $45 \mathrm{mM}$ boric acid, $1 \mathrm{mM}$ EDTA), and $28 \mathrm{~mL}$ of deionized water. The solution was stirred on heat to $37^{\circ} \mathrm{C}$, and filtered through a Whatman No. 1 filter paper. Once cooled, $900 \mu \mathrm{L}$ of $10 \%$ ammonium persulfate and $70 \mu \mathrm{L}$ of TEMED were added to the solution with gentle stirring. The gel was rapidly cast in an SE600 Chroma standard dual cooled gel electrophoresis assembly (Hoefer). Gels were pre-run for 15 minutes using the constant voltage setting at $300 \mathrm{~V}$. Unpurified DNA samples were resuspended in $400 \mu \mathrm{L}$ of deionized water. An equal portion of formamide was added to each sample. Once heated to $55^{\circ} \mathrm{C}$ for 5 minutes, the samples were loaded into the gel. The crude DNA from one CPG support column was loaded into each gel assembly. 
Migration was tracked using Thermo Scientific TriTrack DNA loading dye. The gels were run for approximately 2.5 hours after which, they were imaged on a $20 \times 20 \mathrm{~cm}$ TLC plate $\left(F_{254}\right)$ using a multi-image light cabinet on the epi-UV setting $\left(\lambda_{\mathrm{ex}}=254 \mathrm{~nm}\right)$. Fluorescein labelled DNA was visualized using $302 \mathrm{~nm}$ excitation wavelength. The relevant bands containing the pure DNA were cut from the gel and placed in $50 \mathrm{~mL}$ microcentrifuge tubes. In order to recover the purified DNA, an adaptation of the crush and soak method was used. ${ }^{116}$ The gels were crushed and incubated in deionized water on a New Brunswick Scientific Innova 40 incubator/shaker at $37^{\circ} \mathrm{C}$ for up to 36 hours.

Once extracted, the solution was filtered through $0.22 \mu \mathrm{m}$, low retention, cellulose acetate syringe filters. The filtrate was then freeze-dried on a Labconco Freezone lyophilizer. The solid was resuspended in a minimal amount of deionized water and desalted using Amicon-Ultra $0.5 \mathrm{~mL}, 3 \mathrm{kDa}$ centrifuge units. Purified DNA was quantified by measuring the optical absorbance at $260 \mathrm{~nm}$. Molar extinction coefficients were determined using the nearest neighbour approximation (IDT oligoanalyzer 3.1), taking in to account the $\varepsilon 260$ s of the modifiers used. Oligonucleotide sequences were confirmed using mass spectrometry.

\subsubsection{UV melting temperature studies}

The thermal stability analysis of DNA sequences was performed on a Varian Cary 300 Bio UV-Vis Spectrophotometer equipped with a 6x6 Peltier-thermostated multicell holder. Absorbance was monitored at $260 \mathrm{~nm}$ over a temperature ramp from $20-85^{\circ} \mathrm{C}$. Sample concentrations were determined based on the molar extinction coefficient of the full-length aptamer sequences. For the single stranded sequences, $3 \mathrm{~mL}$ of $0.2 \mu \mathrm{M}$ DNA 
was prepared in $3 \mathrm{M} \mathrm{Mg}$ buffer. Samples containing hybridized aptamer/capture probe were prepared in a 1:2 ratio.

Single stage collection was performed using the following parameters:

Temperature rate was set to $1^{\circ} \mathrm{C} / \mathrm{min}$. or $5^{\circ} \mathrm{C} / \mathrm{min}$. The collection increments were set at $1^{\circ} \mathrm{C}$ with the final hold time of $5 \mathrm{~min}$. was used. Each sample was allowed to cool back down to $20^{\circ} \mathrm{C}$ for $30 \mathrm{~min}$. before the next ramp was recorded. Melting temperature curves were analysed using the dose response curve fitting tool in OriginLab Pro 2017.

\subsubsection{Gel shift assay}

\subsubsection{Hybridization}

In a $1.5 \mathrm{~mL}$ microcentrifuge tube, small aliquots of aptamer and capture were mixed. The samples were made up to $40 \mu \mathrm{L}$. Final concentrations of aptamer and capture probe were $4 \mu \mathrm{M}$. Once vortexed, the samples were placed on a heat block at $95^{\circ} \mathrm{C}$ for 15 mins. They were then allowed to cool to room temperature for 30 mins. and stored at $4^{\circ} \mathrm{C}$ until use (24 hours).

\subsubsection{Non-denaturing gel shift assay}

To prepare the samples for the gel shift assay, $20 \mu \mathrm{L}$ of the hybridized DNA samples and $20 \mu \mathrm{L}$ of $0.1 \mu \mathrm{M}$ of total aflatoxin stock $\left(5: 1: 1: 1 \mathrm{AFB}_{1}, \mathrm{AFB}_{2}, \mathrm{AFG}_{1}, \mathrm{AFG}_{2}\right)$ were placed in a $1.5 \mathrm{~mL}$ microcentrifuge tube and vortexed for 2 hours. Non-denaturing polyacrylamide gels were prepared by mixing $27 \mathrm{~mL}$ of acrylamide solution (acrylamide/bisacrylamide, 40\% solution, 19:1), $18 \mathrm{~mL}$ of $5 \mathrm{X}$ TBE buffer (45 $\mathrm{mM}$ Tris, $45 \mathrm{mM}$ boric acid, $1 \mathrm{mM}$ EDTA), and $44 \mathrm{~mL}$ of deionized water. The solution 
was stirred on heat to $37^{\circ} \mathrm{C}$, and filtered through a Whatman No. 1 filter paper. Once cooled, $900 \mu \mathrm{L}$ of $10 \%$ ammonium persulfate and $90 \mu \mathrm{L}$ of TEMED were added to the solution and gently stirred. The gels were cast and pre-run as previously described for the PAGE purification. The power supply was set to a constant $200 \mathrm{~V}$. Cold water was circulated through the vertical heat exchanger to maintain cool buffer temperatures. Before loading the DNA samples into the gel, equal volumes of $50 \%$ glycerol were added to each sample. The gels were run for approximately 3 hours after which, they were imaged on a $20 \times 20 \mathrm{~cm}$ TLC plate $\left(\mathrm{F}_{254}\right)$ using a multi-image light cabinet on the epi-UV setting $\left(\lambda_{\mathrm{ex}}=254 \mathrm{~nm}\right)$. Fluorescein labelled DNA was visualized using $302 \mathrm{~nm}$ excitation wavelength.

\subsubsection{Circular Dichroism}

Circular dichroism experiments were performed on a Olis Cary-17 spectropolarimeter with circular dichroism module. and analyzing using the inbuilt software. DNA samples were prepared at $3 \mu \mathrm{M}$ in a buffer containing $20 \mathrm{mM}$ Tris, 100 $\mathrm{mM} \mathrm{NaCl}, 5 \mathrm{mM} \mathrm{KCl}, 1 \mathrm{mM} \mathrm{CaCl}$, and $1 \mathrm{mM} \mathrm{MgCl}_{2} .2 \mathrm{~mL}$ of each sample was placed in a $2 \mathrm{~mm}$ path length quartz cuvette. Scans were performed over a range of $220-350$ $\mathrm{nm}$ in $0.75 \mathrm{~nm}$ increments.

\subsubsection{Microscale thermophoresis}

Experiments were performed by 2bind Gmbh as described by Entzian and Schubert (2016) (Regensberg, Germany). Samples were prepared using 3M SELEX buffer (see Table 3.4). A serial dilution of the target was prepared. $4 \mu \mathrm{L}$ of each dilution 
step was mixed with $4 \mu \mathrm{L}$ of the fluorescently labelled aptamer. Capillaries were filled with final reaction mixtures containing individual $\mathrm{AFB}_{1}, \mathrm{AFB}_{2}, \mathrm{AFG}_{1}$, and $\mathrm{AFG}_{2}$ ranging from $1.526 \mathrm{nM}$ to $50 \mu \mathrm{M}$. Aptamers aptamer concentration was kept constant at $5 \mathrm{nM}$. Samples were analyzed on a Monolith NT.115 Pico at $25^{\circ} \mathrm{C}$, with $7 \%$ LED, and $80 \%$ laser power. For each sample, a capillary scan was performed in order to insure the sample was not interacting with the capillary walls. Normalized fluorescence was recorded to ensure no precipitation effects were observed. NanoTemper analysis software that uses the law of mass action fit function to determine the $K_{\mathrm{d}}$ was used for curve fitting. ${ }^{114}$

\subsection{Results and discussion}

\subsubsection{Conformational studies}

\subsubsection{G-quadruplex mapping}

QGRS scoring was computed for each of the six aptamer candidates. The QGRS data reported shows all possible quadruplex locations regardless of overlap. The guanine rich sequences found in DZA3 all coincide with the central PS2.M domain of the aptamer. According to the data in Table 8, the most probable G-quadruplex would occur at position 42 . The results for the DZA 5 aptamer are identical to those of DZA3 indicating that the only probable location for a G-quadruplex falls within the fixed central domain as well. In both cases, the highest G-score obtained is 20. DZA7 is the only sequence that differs slightly. It's highest G-score is 20 , as was found in the other candidates. DZA7 also showed the potential to form low scoring G-quadruplex beyond the PS2.M domain. There are QGRSs reported at positions 47 and 48 (see Table 3.7). 
Table 3.5: Composition and distribution of G-quadruplex forming G-rich sequence DZA3, as predicted by QGRS Mapper

\begin{tabular}{cclc}
\hline Position & Length & QGRS & G-Score \\
\hline 42 & 16 & GGGTAGGGCGGGTTGG & 20 \\
42 & 16 & GGGTAGGGCGGGTTGG & 20 \\
42 & 16 & GGGTAGGGCGGGTTGG & 18 \\
42 & 16 & GGGTAGGGCGGGTTGG & 19 \\
43 & 15 & GGTAGGGCGGGTTGG & 20 \\
43 & 15 & GGTAGGGCGGGTTGG & 20 \\
43 & 15 & GGTAGGGCGGGTTGG & 19 \\
43 & 15 & GGTAGGGCGGGTTGG & 20 \\
\hline
\end{tabular}

Table 3.6: Composition and distribution of Q-quadruplex forming G-rich sequence DZA5, as predicted by QGRS Mapper

\begin{tabular}{cclc}
\hline Position & Length & QGRS & G-Score \\
\hline 42 & 16 & GGGTAGGGCGGGTTGG & 20 \\
42 & 16 & GGGTAGGGCGGGTTGG & 20 \\
42 & 16 & GGGTAGGGCGGGTTGG & 18 \\
42 & 16 & GGGTAGGGCGGGTTGG & 19 \\
43 & 15 & GGTAGGGCGGGTTGG & 20 \\
43 & 15 & GGTAGGGCGGGTTGG & 20 \\
43 & 15 & GGTAGGGCGGGTTGG & 19 \\
43 & 15 & GGTAGGGCGGGTTGG & 20 \\
\hline
\end{tabular}


Table 3.7: Composition and distribution of G-quadruplex forming G-rich sequence DZA7, as predicted by QGRS Mapper

\begin{tabular}{ccll}
\hline Position & Length & QGRS & G-Score \\
\hline 42 & 16 & GGGTAGGGCGGGTTGG & 20 \\
42 & 16 & GGGTAGGGCGGGTTGG & 20 \\
42 & 16 & GGGTAGGGCGGGTTGG & 18 \\
42 & 16 & GGGTAGGGCGGGTTGG & 19 \\
43 & 15 & GGTAGGGCGGGTTGG & 20 \\
43 & 15 & GGTAGGGCGGGTTGG & 20 \\
43 & 15 & GGTAGGGCGGGTTGG & 19 \\
43 & 15 & GGTAGGGCGGGTTGG & 20 \\
47 & 28 & GGGCGGGTTGGTAGAGTGTAGATCTCGG & 8 \\
47 & 28 & GGGCGGGTTGGTAGAGTGTAGATCTCGG & 8 \\
48 & 27 & GGCGGGTTGGTAGAGTGTAGATCTCGG & 7 \\
\hline
\end{tabular}

Each of the random aptamers was analysed using the QGRS mapper. The analysis of the random aptamer candidates did not provide evidence for G-quadruplex binding motifs in any of the aptamers. These and previous results do suggest that the two aptamer templates differ significantly. The DZA sequences have roughly a $10 \%$ higher guanine content than those of the random template.

The QGRS mapper is a useful tool to predict structural binding motifs within the aptamer sequences. Experimental methods for elucidating the structural characteristics of the aptamers will be further discussed in this text. 


\subsubsection{Electrophoretic mobility shift assay}

Based on the selection of structure-switching aptamers, it is theorized that the aptamers in question can adopt two different conformations: a duplexed structure where the aptamer is hybridized to a complementary element in an inhibited state, and a structure associated with the binding of a cognate ligand. ${ }^{57}$ The binding-related conformational changes that occur, can be exploited for sensor applications involving

fluorescent, electrochemical, colorimetric, and various other reporting mechanisms. ${ }^{117,118}$ With this in mind it was important to further characterize switching abilities of the candidate's and to screen them for future applications.

The electrophoretic mobility shift assay (EMSA) is a technique that was originally developed for the study of DNA binding proteins. ${ }^{119}$ The principle of the technique lies in DNA-protein interactions. Changes in molecular weight of DNA complexes can affect their migration through a polyacrylamide gel. If the binding of an aptamer to its small molecule target causes a significant structural change, changes in migration can be visualized in a nondenaturing gel shift assay.

EMSA was used to screen the top five candidates from each template. Aptamers were first hybridized to their fluorescently labelled capture probes in a 1:1 ratio. For each aptamer, one hybridized sample was combined with buffer and one with total aflatoxin mixture. Once incubated, the samples were run on a non-denaturing polyacrylamide gel. The polyacrylamide gels were examined for CP strand displacement. Figure 3.4 shows a graphical representation of the expected gel bands. Only the $\mathrm{CP}$ was fluorescently labelled in this assay, and only two bands should be observable under $302 \mathrm{~nm}$ light. The first being CP, illustrated in lane 1 of Figure 3.4, and the second being the hybridized CP 
+ Apt complex illustrated in lane 2. Lane 3 of Figure 3.4 illustrates the loss of CP + Apt band intensity due to the displacement of $\mathrm{CP}$ when incubated with aflatoxins.

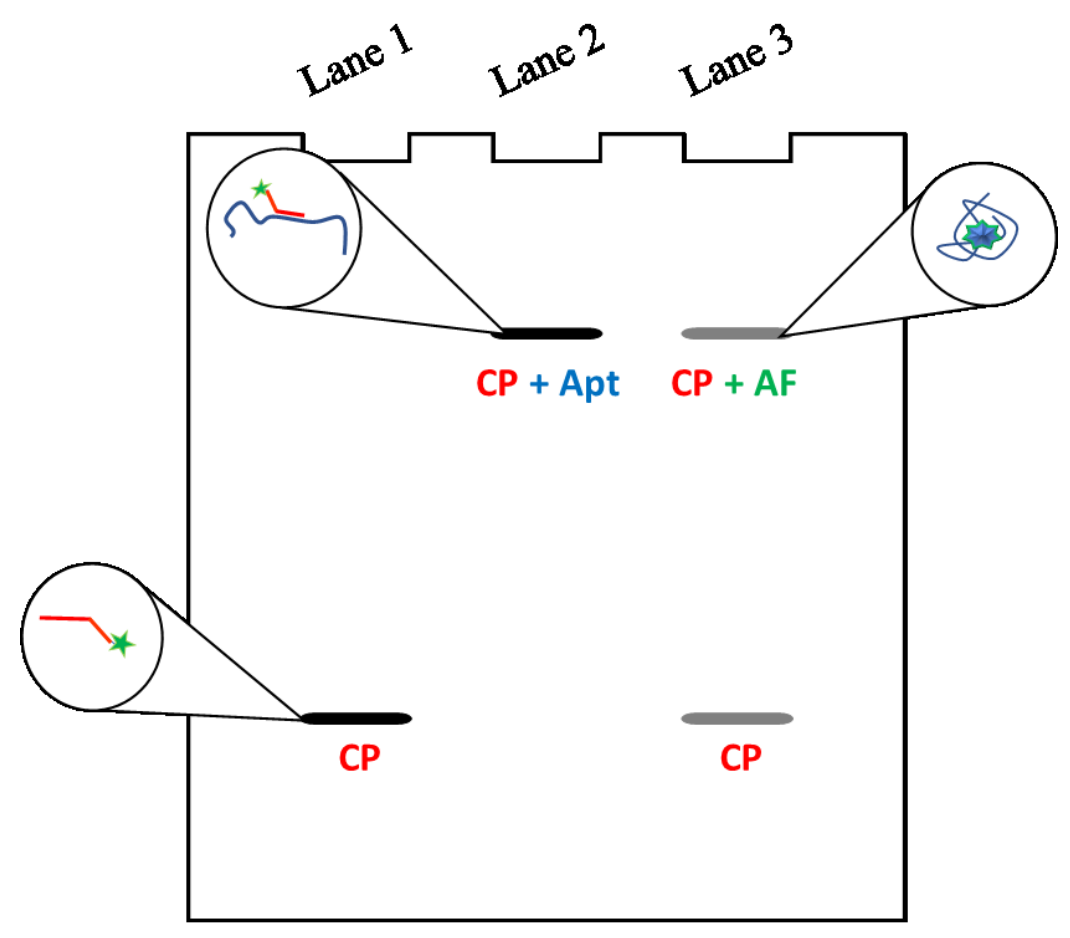

Figure 3.4: Graphical representation of electrophoretic mobility shift assay performed for aptamer candidates. Lane 1: Fluorescently labelled capture probe (CP) control. Lane 2: Hybridized aptamer and fluorescent capture probe. Lane 3: Hybridized complex challenged with aflatoxins causes disassembly of hybridized Apt/CP. The result is a decrease in top band intensity and increase in bottom (CP) band intensity.

Band intensities for each gel were analysed using ImageJ image processing and analysis software. Results for the EMSA screening tests are shown in Figure 3.5 and Figure 3.6. In Figure 3.5 and Figure 3.6, the bottom band intensities representing the $\mathrm{CP}$ are plotted as red bars. The top band intensities are plotted as a line within the bar graph. In each of the figures, the $\mathrm{CP}$ control exhibits a zero value for the top band intensities and a high band intensity for the bottom band. In the case of DZA1 (Lane $2 \& 3$ ), DZA2 (Lane 4 \& 5), and DZA5(Lane $8 \& 9$ ), it is apparent that the aptamers did not hybridize well (Figure 
3.5). As in the CP control lanes, the highest fluorescence intensity is observed in the bottom band. This is further supported when observing the gel under the epi-UV $\left(\lambda_{\mathrm{ex}}=\right.$ $254 \mathrm{~nm}$ ) setting in the imaging cabinet (Appendix B), in which there is no band that coincides with the annealed aptamer-CP complex. This does not appear to be the case for two of the DZA candidates. DZA3 (Lane $6 \&$ 7) and DZA 7 (Lane $11 \& 12$ ) show significant decreases in top band intensities (38\% and $60 \%$, respectively) when challenged with aflatoxins. This is likely attributed to the displacement of CP upon ligand binding. An increase in bottom band intensity was anticipated for the positive strand displacement results, however, very slight changes in band intensity were hard to record due to the high background. DZA7 did show a slight increase in bottom band intensity which was expected to occur during binding.
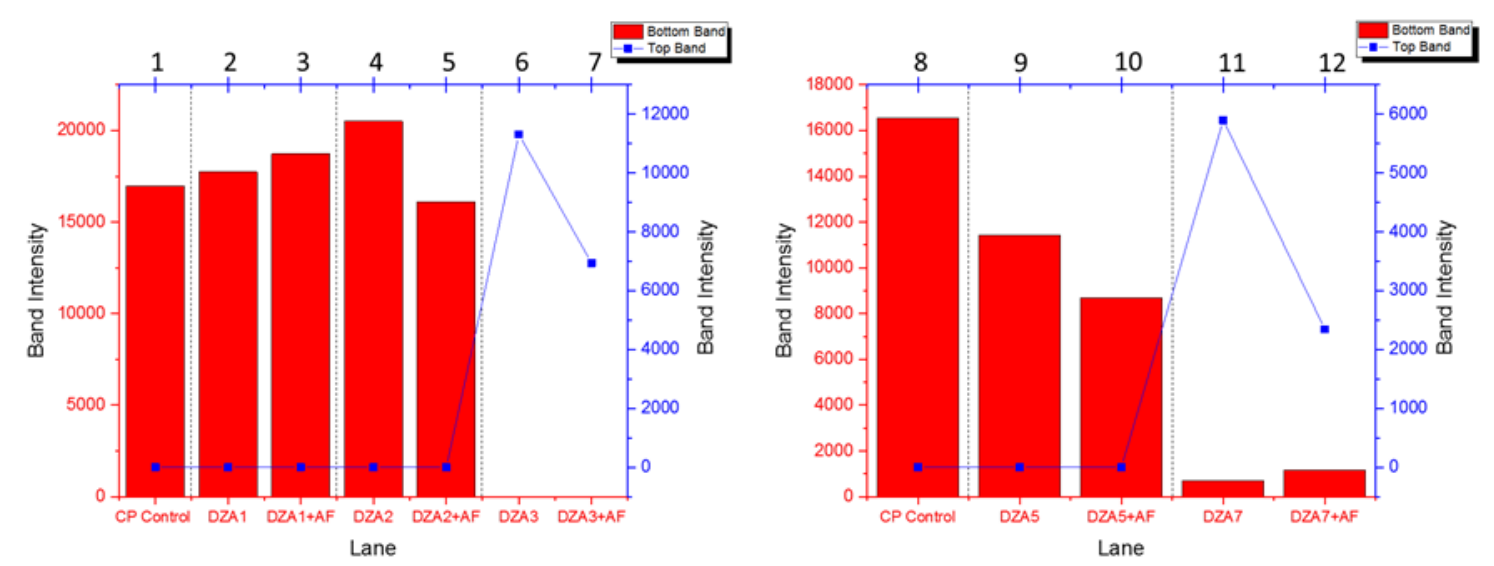

Figure 3.5: EMSA results from preliminary DZA aptamer candidate screening for total aflatoxin mixture. Red bars represent bottom CP band intensity. The blue line represents the change in top CP + Apt band intensity.

When analysing the ImageJ results for the random aptamer candidates, most of the sequences hybridized with higher efficiencies than those of the DZA candidates (see Figure 3.6). 3MR1 had the lowest observed hybridization as seen in Lane 2 of Figure 3.6. 
The epi-UV images also show an observable amount of unhybridized 3MR1 aptamer in Lanes 2 and 3. Lane 3 shows significant strand displacement based on the decrease in top band intensity. The decrease in top band intensity caused by the addition of aflatoxins does seem promising (37\%) even though the hybridization was inefficient. 3MR2 and 3MR4 both showed efficient hybridization of the aptamer and random CP. Neither showed discernable decreases in top band intensities on the other hand. The ImageJ analysis showed a slight $1 \%$ decrease in top band intensity for 3MR2. 3MR3 appeared to hybridize efficiently and had a decrease top band intensity decrease of 33\%. Finally, the 3MR5 aptamer displayed slightly higher bottom band intensity. The CP strand displacement resulted in a decrease of roughly $13 \%$ for 3MR5's top band.
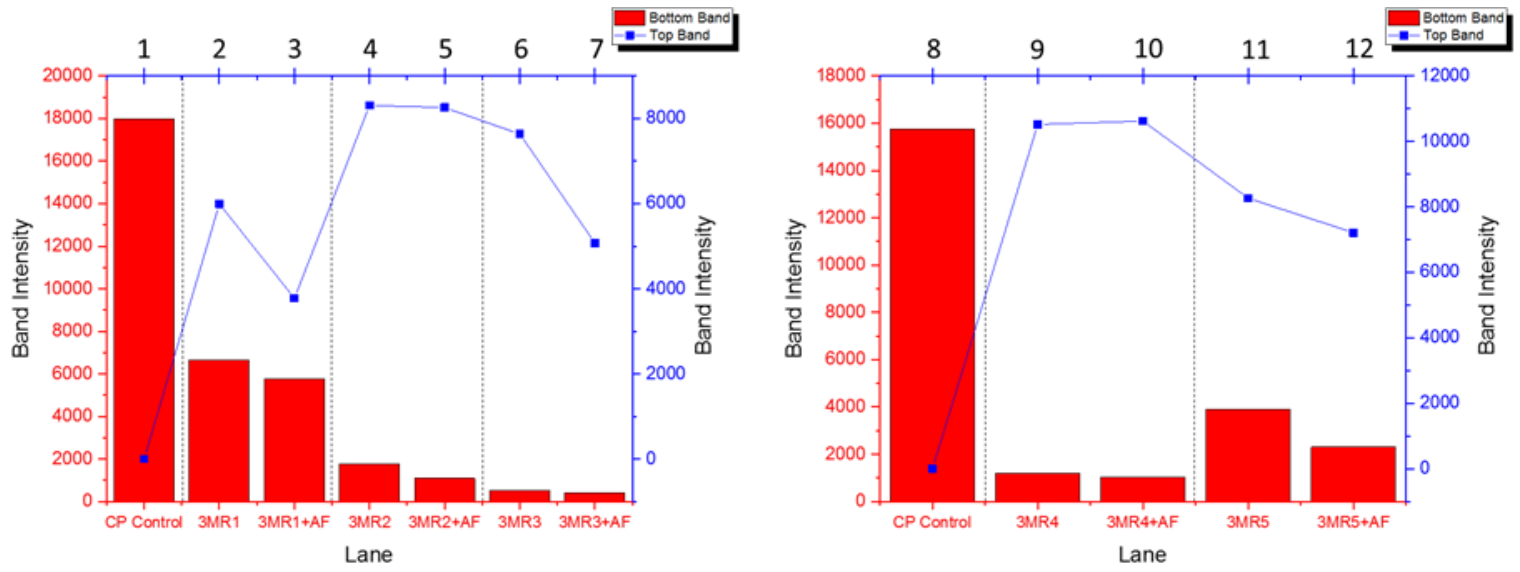

Figure 3.6: EMSA results from preliminary 3MR aptamer candidate screening for total aflatoxin mixture. Red bars represent bottom $\mathrm{CP}$ band intensity. The blue line represents the change in top $\mathrm{CP}$ + Apt band intensity.

It is important to note that when performing the analysis with the ImageJ tool, lowintensity bands are difficult to assess since they are sometimes concealed by background fluorescence. For this reason, small increases in bottom band intensity are often not 
observed after stand displacement. This method was utilized as a qualitative screening method for the aptamer candidates. To save time, a total of three aptamers from each template were chosen for further testing and dissociation constant determination. Of the DZA aptamers, DZA3 and DZA7 were chosen due to their apparent hybridization efficiencies and their strand displacement results. DZA5 was the third candidate to be chosen. The results for DZA5 were less promising. Low bottom band intensities in Lanes 9 and 10 may suggest that there was some degree of hybridization and that the lanes were subject to high background fluorescence due to streaking. This is further supported in Appendix B.1. Of the 3MR aptamers, 3MR3 and 3MR5 were the top 2 candidates. The third candidate to be considered was 3MR2. 3MR2 did have a much lower decrease in top band intensity, however, it did boast a higher apparent degree of hybridization and as a result, would form a more stable duplex with its complementary CP.

\section{Preliminary specificity screening using EMSA}

Following the structure switching gel shift assays, EMSAs were performed to determine the preliminary selectivity of the aptamers. Candidates were screened for cross-reactivity against $0.05 \mu \mathrm{M}$ OTA, DON, FB1, and ZON. Upon initial inspection of the fluorescent images, there does not appear to be a significant decrease in top band intensity for either the DZA or the 3MR candidates. The gel images were again analysed using the ImageJ software. Differences in top band and bottom band intensities were recorded and compared. DZA3 had varying top band intensities when compared to the controls. The lane that was challenged with FB1 appears to have a large decrease in top band intensity. This could indicate the cross-reactivity of DZA3 with FB1. Further 
analysis of the Apt. band from the epi-UV image, suggest that the low top band intensity is not a result of the strand displacement reaction. In all lanes, the Apt band intensities were lower than that of the control Apt band. Results for DZA5 show a decrease in top band intensity in the OTA lane. Again, when analysing the epi-UV gel images, there does not appear to be noticeable changes in $\mathrm{CP}+$ Apt and Apt bands when compared to the control lane. DZA5 does not appear to have hybridized as well as the other aptamers. Evidence of this is seen in the high background of all lanes including the hybridized control. DZA7 showed decreases in band intensity for all counter-toxins. The Apt band intensities follow the same trend as the top band intensities indicating that the low band intensities could again be attributed to poor hybridization. It is thought that the DZA aptamers may form less stable duplex due to their propensity for forming high order folding motifs. The presence of the G-quadruplex domain could result in enhanced structure switching properties at the expense of duplex stability.
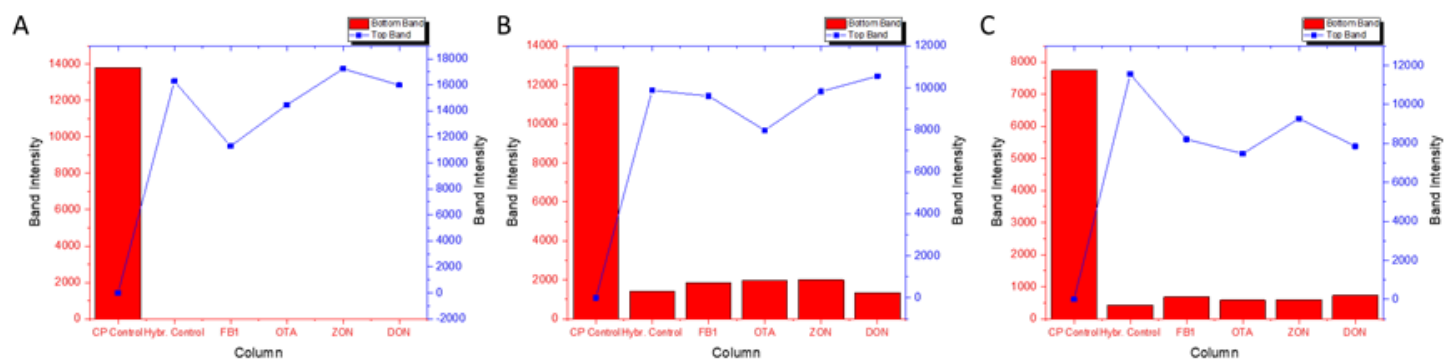

Figure 3.7: EMSA results from preliminary selectivity screening with counter toxins FB1, OTA, ZON, and DON for aptamers DZA3 (A), DZA5 (B), DZA7 (C). Red bars represent bottom CP band intensity. The blue line represents the change in top $\mathrm{CP}+\mathrm{Apt}$ band intensity. 

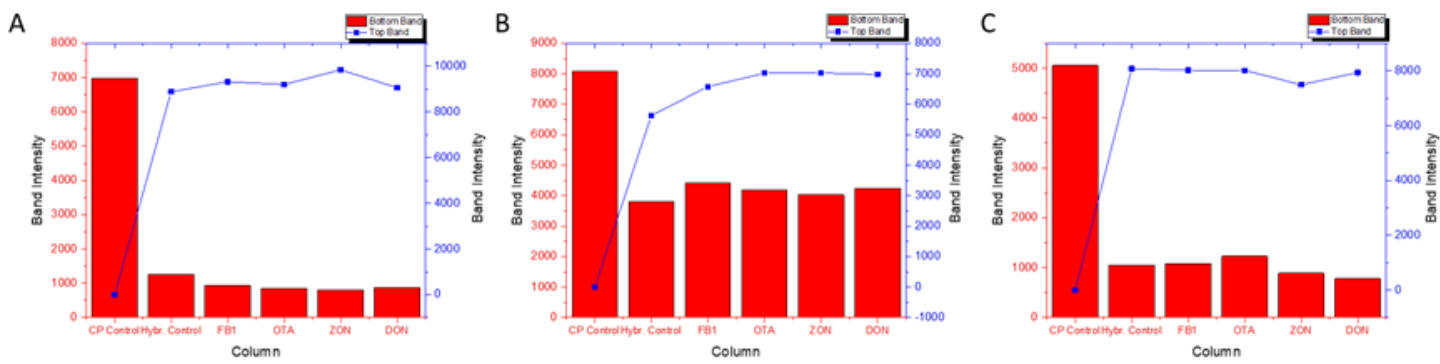

Figure 3.8: EMSA results from preliminary selectivity screening with counter toxins FB1, OTA, ZON, and DON for aptamers 3MR2 (A), 3MR3 (B), 3MR5 (C). Red bars represent bottom CP band intensity. The blue line represents the change in top $\mathrm{CP}+\mathrm{Apt}$ band intensity.

The random aptamers exhibited more consistent results. 3MR2, 3MR3, and 3MR5 all had top band intensities comparable to that of their respective hybridized controls. Upon visual examination, and analysis by ImageJ it appears the random aptamers did not hybridize consistently. There is little evidence, however, that the counter-toxins caused significant strand displacement. The random candidates are likely selective.

The EMSAs provide preliminary information on both the structure-switching ability and the selectivity of the aptamer candidates. It would be beneficial however to test the aptamer candidates using a method that does not depend on the signalling of an antisense strand. The results of the gel shift assay can be somewhat ambiguous. Poor hybridization of the complementary sequences can suggest that the strand displacement is occurring due to ligand-binding even if this is not the case. Extra care must also be taken when running a nondenaturing gel. Elevated temperatures within the gel apparatus could have accounted for some of the discrepancies.

The intention was to use the EMSA as a preliminary screening tool to further narrow the list of possible aptamers for more in-depth characterization. Therefore, no definitive conclusions were made based on the apparent affinity and selectivity of the aptamers. The results do support that the selection process produced aptamers that contain some 
structure switching properties. To further confirm the affinity and selectivity of the aptamers a method of testing that does not involve the use of a complementary $\mathrm{CP}$ will be discussed in section 2.4.2.

\subsubsection{Thermal stability}

To further support the predicted structural analysis of the aptamers, a thermal stability test was performed by recording ultraviolet DNA melting curves for the aptamer candidates. The melting temperature $\left(\mathrm{T}_{\mathrm{m}}\right)$ analysis of aptamers can provide insight into the stability and conformation of folded nucleic acid structures. The ultraviolet absorption of a polynucleotide is significantly less than that of the sum of its constituent nucleotides. ${ }^{120}$ Moreover, the absorption of a structure-stabilized oligonucleotide is reduced even further due to excitonic state localization observed in dual stranded DNA. ${ }^{121}$ The hyperchromic effect is described as the experimentally observed increase in absorption ( $25-30 \%$ ) during DNA denaturation. ${ }^{121}$ The $\mathrm{T}_{\mathrm{m}}$ of an oligonucleotide sequence is taken as the midpoint in the hyperchromic transition, where the DNA 
undergoes a conformation transition from folded to unfolded or double-stranded to single-stranded.

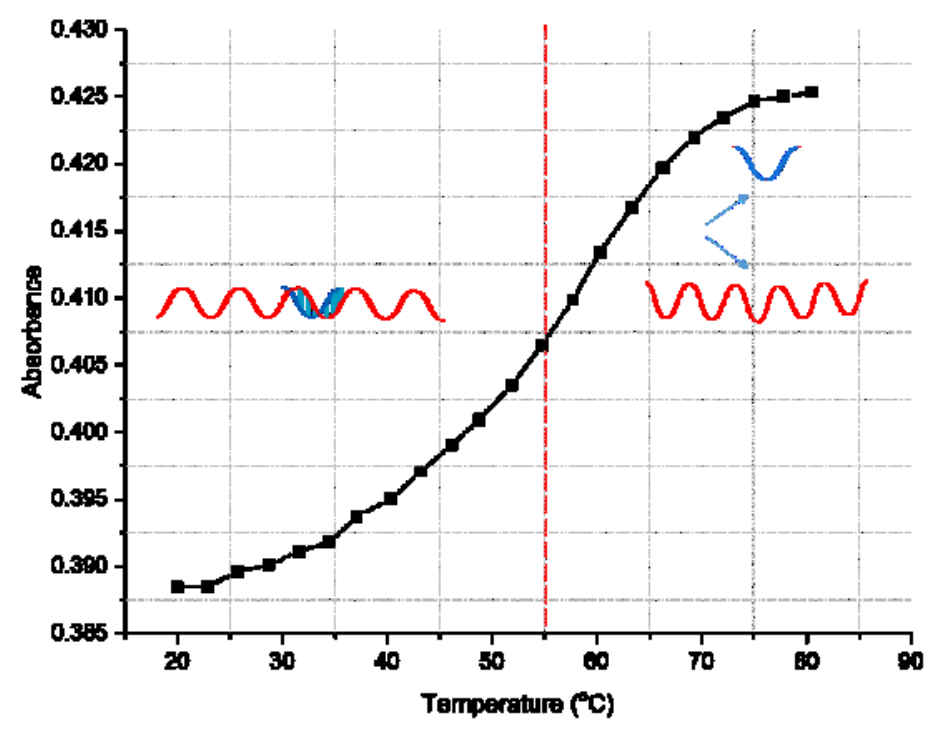

Figure 3.9: Schematic representation of the thermal denaturation of duplexed DNA. The UV melting temperature curve demonstrates that the hyperchromic effect is proportional to the rate of denaturation. The melting temperature is taken as the midpoint in the hyperchromic transition, where the amount of ssDNA is of equal proportion to that of the dsDNA in solution

The Single stranded DNA melting temperatures that were obtained experimentally displayed monophasic curves with varying cooperativities based on the complexity and stability of their structures. Secondary structure predictions were also modelled for each of the final aptamer candidates. Structure prediction software like UNAFold approximates nucleic acid folding, hybridization and $\mathrm{T}_{\mathrm{m}} \mathrm{s}$ using corrected parameters. For these experiments, folding temperature was set to $25^{\circ} \mathrm{C}$, and DNA concentration was set to $0.2 \mu \mathrm{M}$. The $\mathrm{Na}^{+}$and $\mathrm{Mg}^{2+}$ concentrations were adjusted to $100 \mathrm{mM}$, and $1 \mathrm{mM}$ respectively, to mimic the salinity of the buffer used during the $T_{m}$ studies. The three aptamer candidates from the DZA library; DZA3, DZA5, and DZA7 had experimental $\mathrm{T}_{\mathrm{m}} \mathrm{s}$ of $45.5 \pm 0.2^{\circ} \mathrm{C}, 83.6 \pm 3.0^{\circ} \mathrm{C}$, and $51.9 \pm 0.3$ respectively. Table 3.8 compares the 
experimental $\mathrm{T}_{\mathrm{m}} \mathrm{s}$ and predicted melting temperatures obtained from UNAFold. DZA5's $\mathrm{T}_{\mathrm{m}}$ differs greatly from the predicted value. In the case of the DZA candidates, the trend in melting temperature does, however, follow the apparent degree of secondary structure. As seen in Figure 3.10 DZA5 appears to have the highest degree of secondary structure while DZA 3 and DZA7 have comparable folding patterns. These observations are primarily based on the presence and size of loops, bulges, internal loops, and hairpin motifs within the predicted structures. While it is not entirely known if the large single stranded loops or the duplexed portions form the binding regions of the aptamer, DZA 3 and DZA 7 both have very similar secondary structures, and their central PS2.M domains are involved in a region with relatively high complementarity. This may provide more insight into the binding characteristics of the aptamers when tested via other binding assays.

Table 3.8: Melting temperatures for oligonucleotide sequences

\begin{tabular}{ccc}
\hline Sequence & Predicted $\mathbf{T m}^{*}\left({ }^{\mathbf{0}} \mathbf{C}\right)$ & Experimental $\mathbf{T m}\left({ }^{\mathbf{0}} \mathbf{C}\right)$ \\
\hline DZA3 & 49.3 & $45.5 \pm 0.2$ \\
DZA5 & 54.2 & $83.6 \pm 3.0$ \\
DZA7 & 46.3 & $51.9 \pm 0.3$ \\
\hline 3MR2 & 48.9 & $39.2 \pm 0.4$ \\
3MR3 & 48.7 & $45.0 \pm 1.7$ \\
3MR5 & 51.0 & $53.3 \pm 3.6$ \\
\hline
\end{tabular}

*Predicted melting temperatures determined using UNAFold (Licensed to Integrated DNA Technologies) 


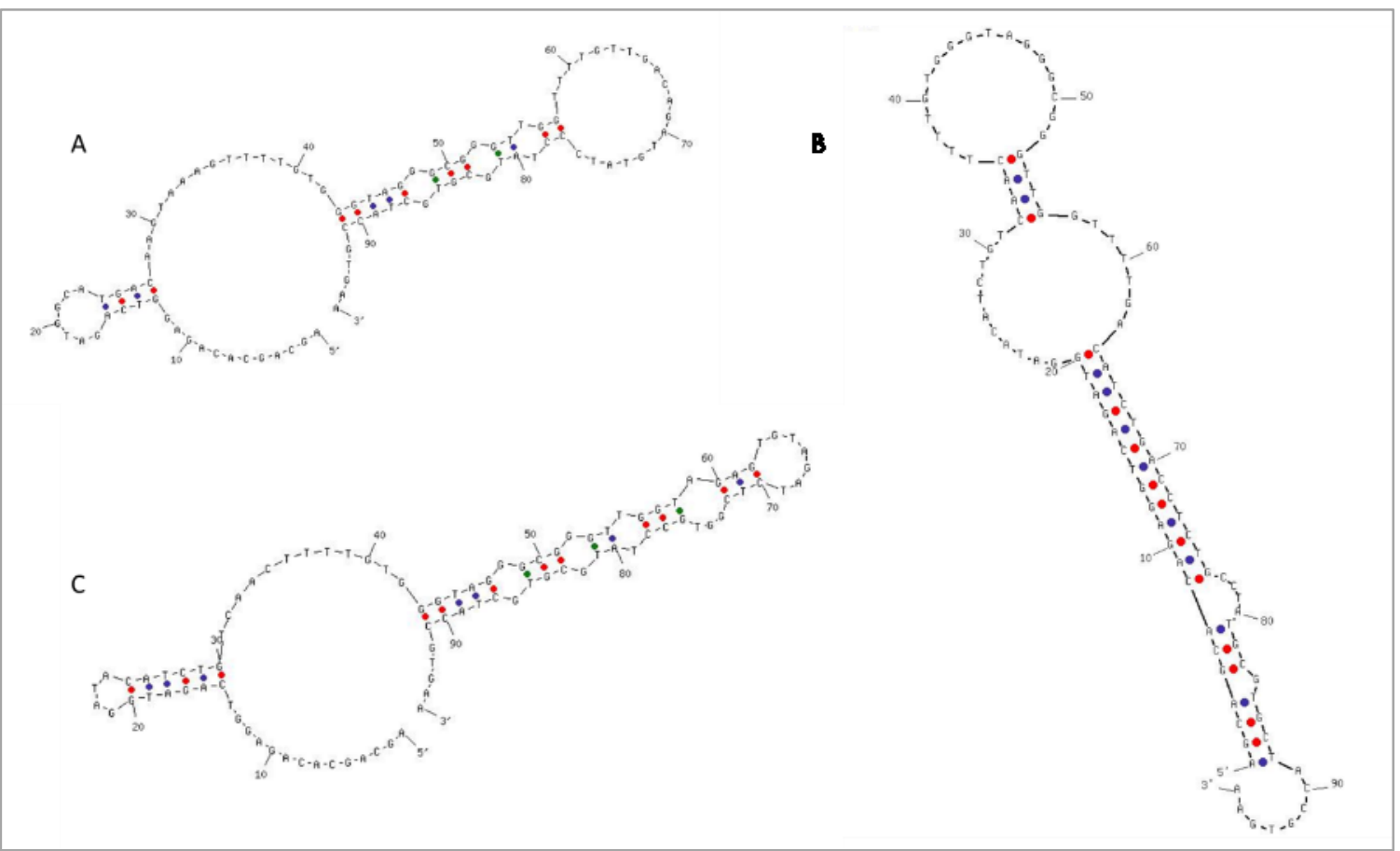

Figure 3.10: Secondary structure prediction for A) DZA3, B) DZA7, and C) DZA5 sequences. Image generated by UNAFold.

In the case of the $3 \mathrm{MR}$ aptamer candidates, all melting temperature curves were monophasic. Aptamers 3MR2, 3MR3, and 3MR5 had $\mathrm{T}_{\mathrm{ms}}$ of $39.2 \pm 0.4,45.0 \pm 1.7$, and $53.3 \pm 3.6$, respectively. Both the experimental and predicted $\mathrm{T}_{\mathrm{ms}}$ were within the range of the DZA $T_{m} s .3 M R 5$ had the highest predicted and experimental $T_{m}$. Both results were comparable. 3MR2 and 3MR3 had almost identical predicted $\mathrm{T}_{\mathrm{m}} \mathrm{s}$, however, their experimental $\mathrm{T}_{\mathrm{ms}}$ differed by roughly $6^{\circ} \mathrm{C}$ (see table 8 ). When looking at the secondary structure predictions, they appear to support that 3MR3 could have a slightly higher melting temperature due to the degree of folding. Upon initial assessment, both the predicted structures of 3MR2 and 3MR3 look very similar. One noticeable difference in the structure is the presence of a tight hairpin loop and internal loop. 3MR2, on the other hand, contains a much larger stem loop within the same region. This suggests that the 
predicted secondary structures that show higher degrees of folding are associated with higher melting temperatures.

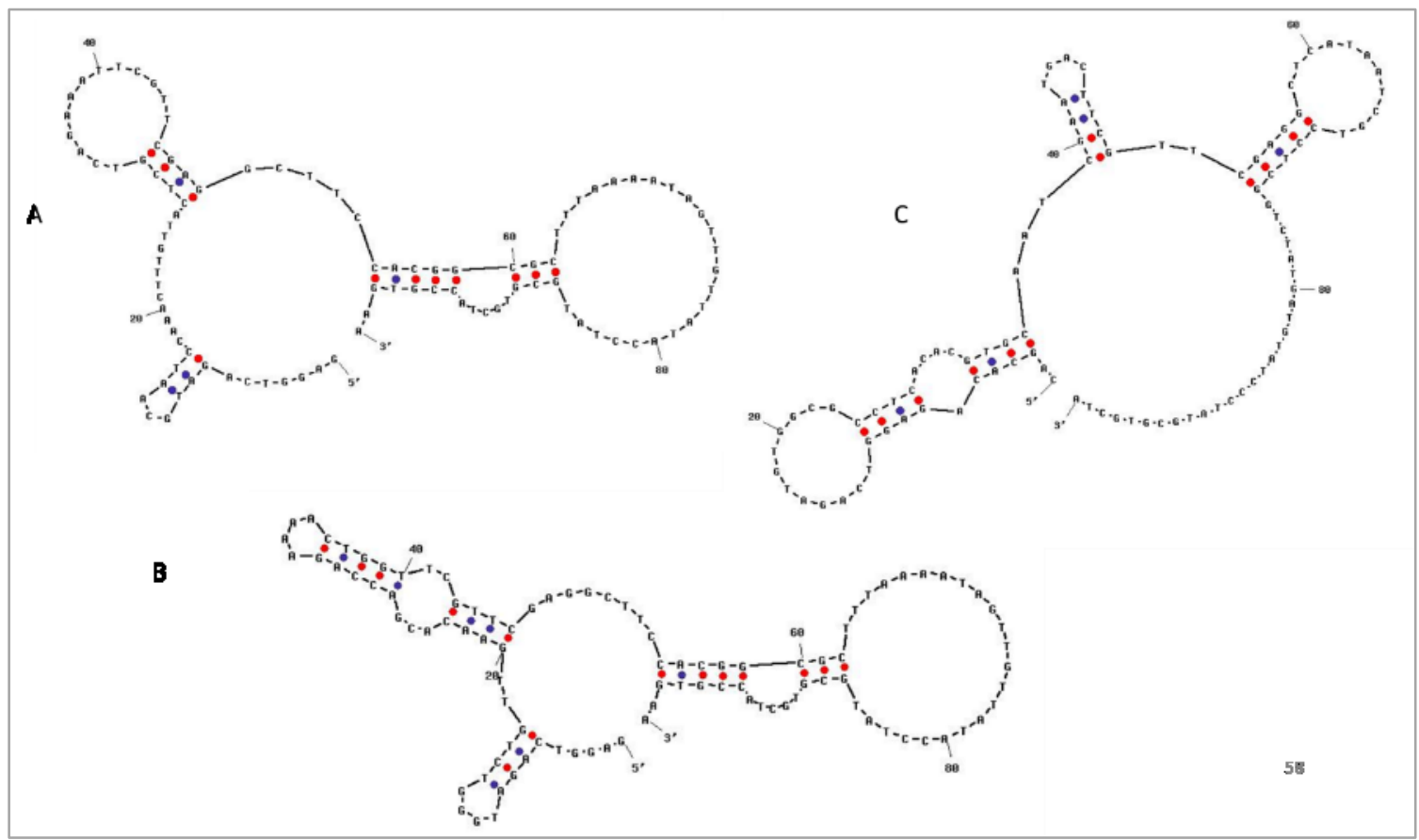

Figure 3.11: Secondary structure prediction for A) 3MR2, B) 3MR3, and C) $3 M R 5$ sequences. Image generated by UNAFold.

When investigating structure predictions for aptamers, it is important to remember that software is limited to modelling two-dimensional folding, and therefore cannot predict higher order structures such as G-quadruplexes and other three-dimensional binding motifs. Melting temperature studies, on the other hand, can be utilized for the elucidation of higher order structures in DNA. Changes in absorbance due to Gquadruplex melting can be monitored at $295 \mathrm{~nm}$. While the absolute absorbance at 295 $\mathrm{nm}$ is not comparable to the absorption maximum of DNA at $260 \mathrm{~nm}$, Mergny and Lacroix (1998) reported a change in absorbance at $295 \mathrm{~nm}$ that ranged from $50-80 \%$ when denaturing G-quadruplex structures. ${ }^{122,123}$ Short quadruplex containing sequences exhibit a decrease in absorbance with increasing temperature. This is shown in Figure 
3.12, where the PS2.M, G-quadruplex containing sequence shows higher absorbance at $295 \mathrm{~nm}$ due to the presence of a more ordered binding motif. The denaturing of the sequence results in hypochromicity since the G-quadruplex structure has a higher optical absorbance than that of the unstacked bases. This is often the case in relatively short sequences (PS2.M being 18-nt in length). In the case of both the DZA and 3MR aptamer candidates, the same melting profile is not observed. This is purportedly due to the length of the sequences. The number of bases outweighs the absorbance of the G-quadruplex when denatured.

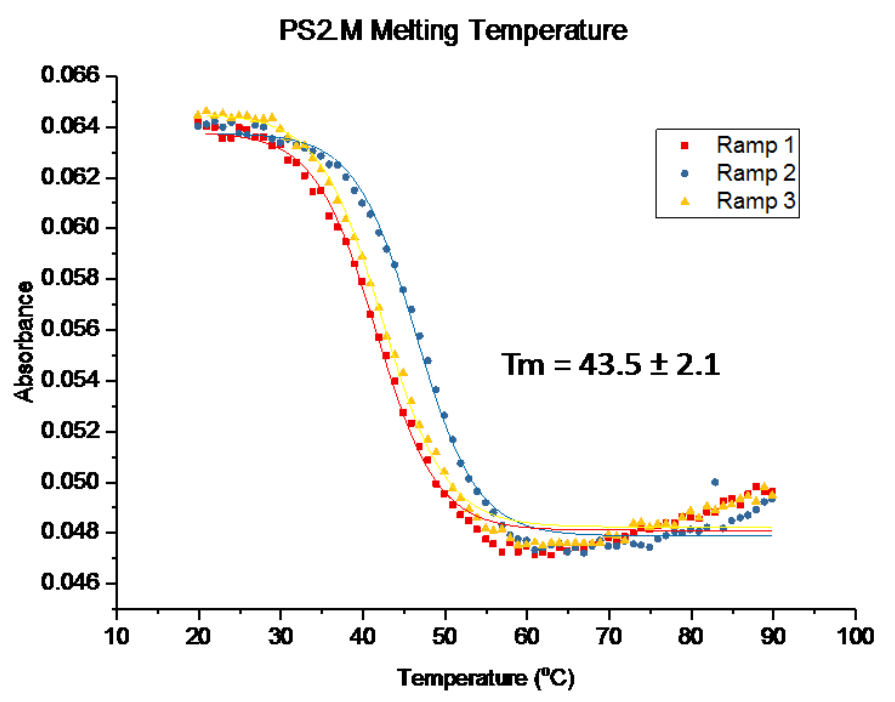

Figure 3.12: Melting temperature curves for PS2.M G-quadruplex forming sequence (295 $\mathrm{nm})$. Hyperchromicity observed at low temperatures suggests the presence of higher order structures.

Figure 3.13 and Figure 3.14 demonstrate the difference in melting temperature profiles at $295 \mathrm{~nm}$ between the DZA and random aptamers.

The melting temperature curve of DZA3 contains a "hump" near $40^{\circ} \mathrm{C}$. The subsequent decrease in absorbance at $295 \mathrm{~nm}$ as the temperature increases is indicative of G- 
quadruplex melting. The hypochromicity resulting from G-quadruplex melting is however superimposed by an overall increase in absorbance due to the overlying hyperchromic effect. This increase suggests that the sequence is stabilized by higher order structures at low temperatures. As the DNA strand is denatured, the loss of hydrogen bonds in duplexed portions and decreased base stacking still causes a substantial increase in absorption. 3MR3 is used as an example in Figure 3.14. In contrast, 3MR3 does not show any increased absorbance at lower temperatures when the melting curve is recorded at $295 \mathrm{~nm}$. The melting temperature profiles are similar to those recorded at $260 \mathrm{~nm}$ and do not suggest that there is are any structures present that exhibit high absorbance.

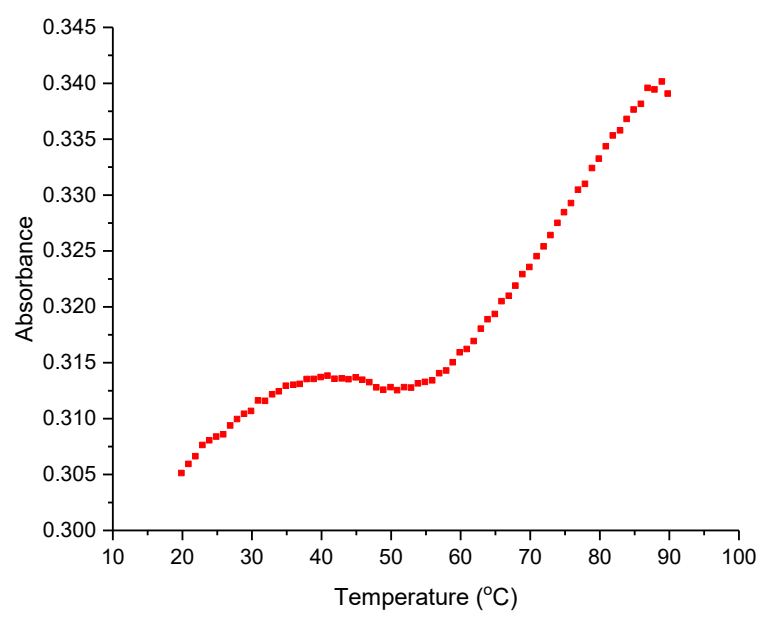

Figure 3.13: Example of melting temperature profile from DZA templates (DZA3). The decrease in intensity resulting from G-quadruplex melting is masked by an overlying increase in absorbance caused by the hyperchromic effect. The resulting "hump" in the spectrum is not present in the $3 M R$ candidates 


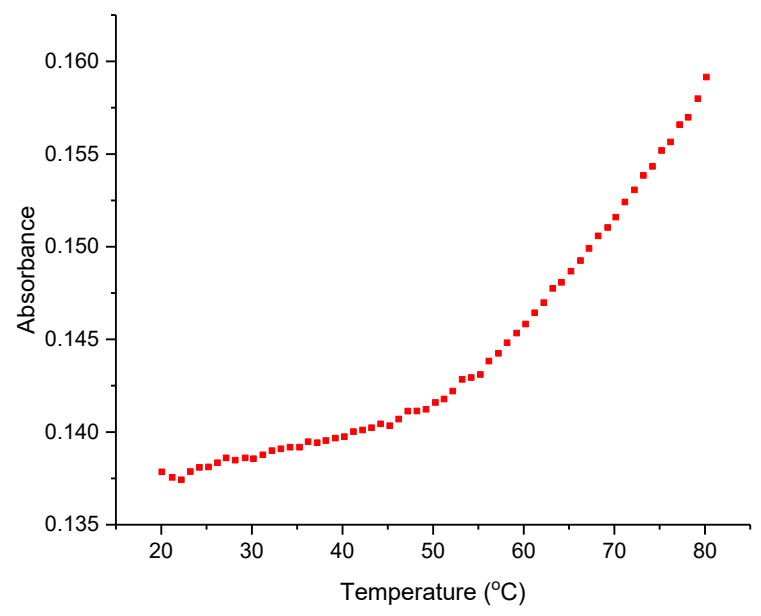

Figure 3.14: Example of melting temperature profile random template (3MR2). The sequence does not exhibit increased absorbance at low temperatures, suggesting there is no G-quadruplex formation.

\subsubsection{Circular Dichroism}

CD spectra were recorded for each of the six aptamer candidates to help support the presence of structural differences between the two candidate templates. The PS2.M sequence was also studied under the same conditions and used as a comparison.

Figure 3.15 shows the recorded CD spectrum of the PS2.M sequence. Within the spectral range of interest, the short 18-nt sequence exhibits a maximum at $\sim 265-270 \mathrm{~nm}$ and a negative band at $\sim 245 \mathrm{~nm}$. This suggests the presence of a parallel G-quadruplex. Furthermore, the dichroic signals coincide with the signatures of a potassium stabilized parallel G-quadruplexes 1-1XAV, which has a repeating G-rich sequence $\mathrm{d}\left(\mathrm{AG}_{3} \mathrm{TG}_{3} \mathrm{~T}\right)$ that is analogous to that of PS2.M. ${ }^{112}$ Risitano and Fox report the use of non-nucleosidic linkers such as propanediol and hexaethylene glycol among $\mathrm{G}_{3}$-tracts, to stabilize the 
formation of parallel G-quadruplexes. These too generate very similar CD signatures, with ellipticity a maximum at $\sim 264 \mathrm{~nm}$ and a minimum at $\sim 244 \mathrm{~nm} .{ }^{124}$

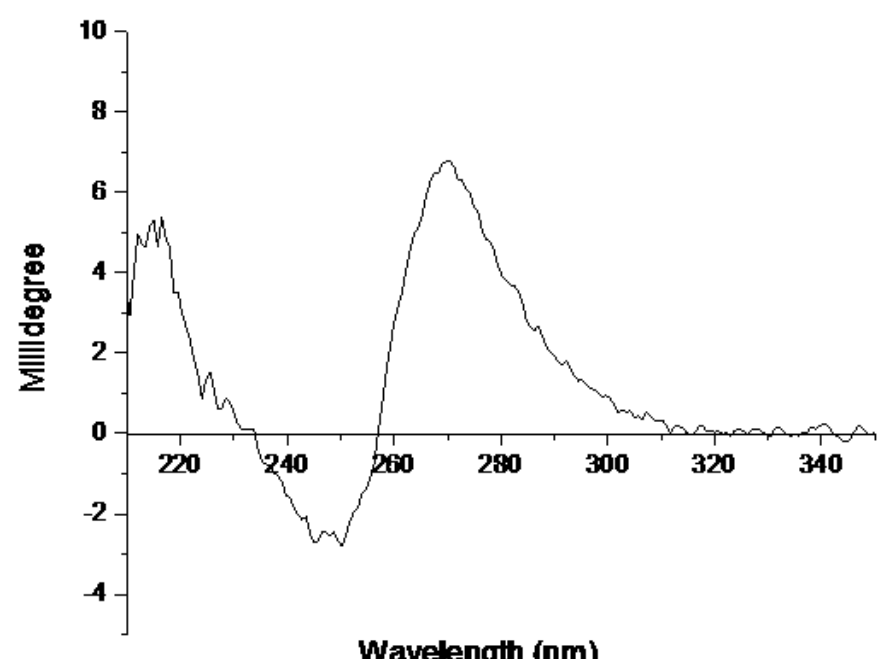

Figure 3.15: CD spectrum of parallel-stranded G-quadruplex forming PS2.M sequence in $20 \mathrm{mM}$ Tris buffer (100 mM NaCl, $\left.5 \mathrm{mM} \mathrm{KCl,} 1 \mathrm{mM} \mathrm{CaCl}_{2}, 1 \mathrm{mM} \mathrm{MgCl}_{2}\right)$, as monitored by $\mathrm{UV}-\mathrm{CD}$ using an Olis Cary 17 CD spectropolarimeter.

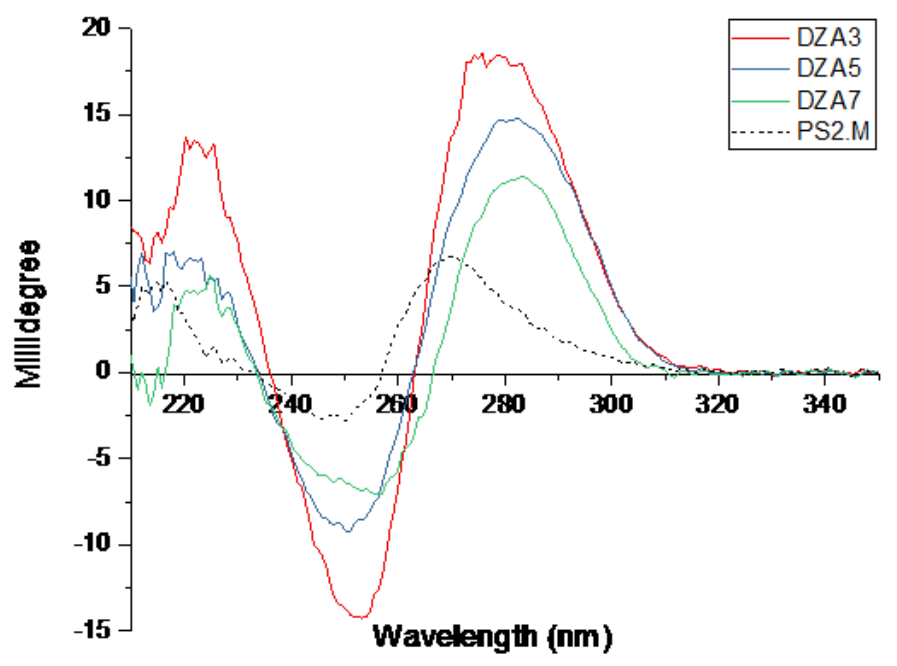

Figure 3.16: CD spectrum of DZA aptamer candidate sequences in $20 \mathrm{mM}$ Tris buffer $(100 \mathrm{mM}$

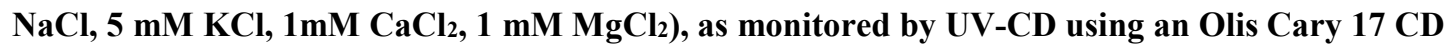
spectropolarimeter. 
DZA aptamer candidates show slight shifts in maxima (see Figure 3.16). Initially, it was postulated that parallel to antiparallel G-quadruplex transitions were occurring due to conformational changes that arise from destabilization caused by the intramolecular duplex formation in the elongated sequences. Further analysis did not support this notion, however. CD spectra belonging to antiparallel G-quadruplexes typically exhibit a maximum equal to or higher than $295 \mathrm{~nm}$. None of the DZA aptamer candidates produced ellipticity maxima that high. In comparison to the $3 \mathrm{MR}$ CD spectra, the DZA maxima have slightly squared peaks, and are slightly shifted towards a lower wavelength. Smaldino et al. have reported human telomeric sequences that produce unimolecular mixed parallel/antiparallel strand orientations. G-rich sequences that contain successive parallel and antiparallel structures produce spectra with overlapping maxima. ${ }^{125}$ This results in both a merging of the two peaksand a decrease in amplitude. Based on previous characterizations it is unlikely that multiple quadruplexes are forming. QGRS mapping suggests that the only quadruplex forming regions are within the short central domain. Due to similar base stacking interactions, the $\mathrm{CD}$ spectrum of a parallel G-quadruplex is very similar to that of an A-form helical conformation. Both profiles are characterized by an ellipticity maximum at roughly $265 \mathrm{~nm}$, a transition near $260 \mathrm{~nm}$, and a minimum at $245 \mathrm{~nm}$. Herein, it is suggested that the presence of parallel G-quadruplexes in DZA 3 and DZA5 primarily is overlapped by a signal produced by the higher degree of duplexed DNA within the aptamer structures. Differences between parallel quadruplexes and helical structures do arise below $220 \mathrm{~nm}$ where A-form helical DNA produced a negative band centred towards $210 \mathrm{~nm}$. Below $220 \mathrm{~nm}$ the 3MR sequences do all show lower negative values than the DZA candidates which would suggest the presence of helical 
duplexed structures. However, the CD spectra can only be reliably analysed from 220$350 \mathrm{~nm}$ since the Tris containing buffer shows strong positive bands below $220 \mathrm{~nm} .{ }^{126}$ This can be seen in Appendix D.1.

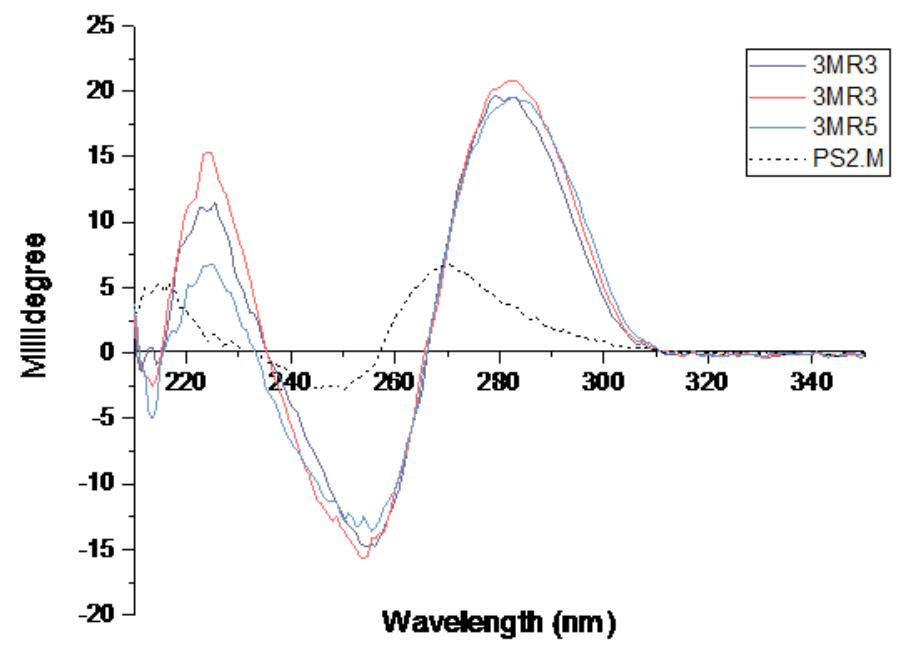

Figure 3.17: CD spectrum of 3MR aptamer candidate sequences in $20 \mathrm{mM}$ Tris buffer $(100 \mathrm{mM}$ $\mathrm{NaCl}, 5 \mathrm{mM} \mathrm{KCl}, 1 \mathrm{mM} \mathrm{CaCl} 2,1 \mathrm{mM} \mathrm{MgCl}$ ), as monitored by UV-CD using an Olis Cary $17 \mathrm{CD}$ spectropolarimeter.

In order to fully characterize these systems, it would have been beneficial to explore temperature dependent ellipticity measurements, as they may have helped determine secondary structures within the relatively long aptamers. This as well as ligand binding could trigger changes in spectral properties due to the disruption of base stacking. Alternatively, ethanol has been shown to be a potent G-quadruplex inducer. ${ }^{127}$ Given the long aptamer sequences, ethanol solutions could be utilized to uncover underlying Gquadruplex structures. 


\subsubsection{Dissociation constant $\left(K_{d}\right)$ determination using microscale thermophoresis}

Aptamer candidates were tested by MST to determine their potential for in-

solution biorecognition assays. For each sample, capillary scans were performed to

ensure that there were no non-specific interactions between the biomolecules and capillary tubes (see Figure 3.18).

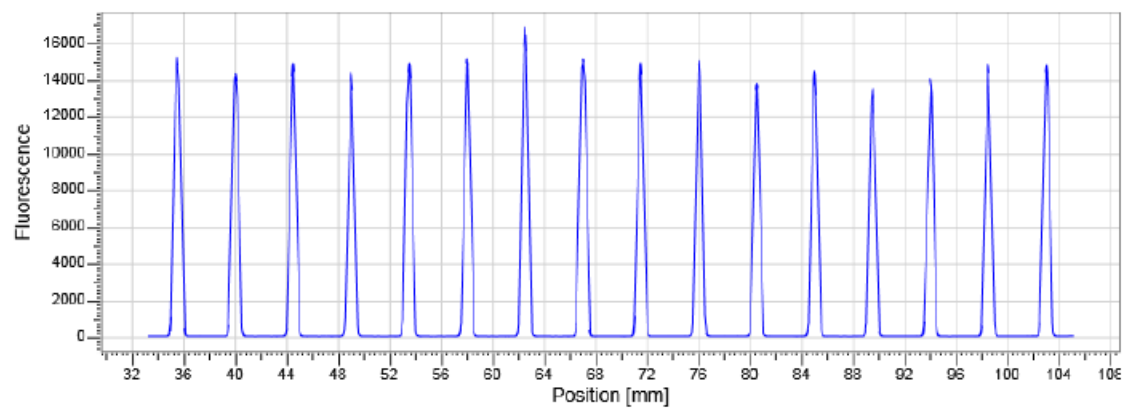

Figure 3.18: Successful capillary scan for DZA3 samples. Non-specific adsorption of sample onto the capillary wall is normally demonstrated by irregularly shaped fluorescence peaks along the capillary

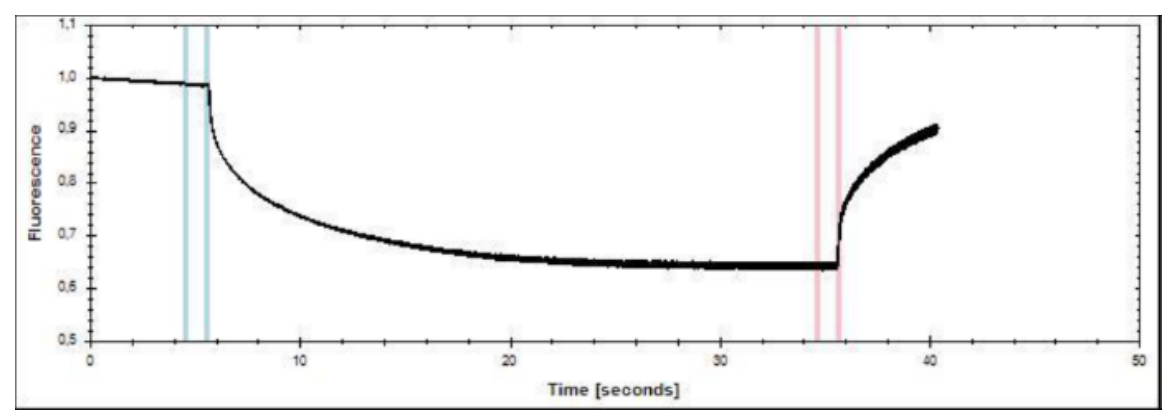

Figure 3.19: Normalized fluorescence measurement for Cy5 labelled DZA3. Performed in order to ensure there is no precipitation or aggregation effect. The figure represents an MST time trace. The initial cold phase (green) shows the sample fluorescence with the laser off. The laser is switched on after the 5 second and a temperature gradient is established. A decrease in fluorescence is observed due to thermophoretic movement (red). After 30 seconds, the laser is turned off and the molecules diffuse back.

Background fluorescence for each sample was measured to ensure there were no irregular jagged traces caused by precipitation or molecule adsorption to the capillary walls 
(Figure 3.19). Initially, each of the aptamer candidates was tested against $\mathrm{AFB}_{1}$. Of the six chosen candidates, DZA3 and 3MR5 were shown to bind $\mathrm{AFB}_{1}$ with high affinity. Their $\mathrm{K}_{\mathrm{d}}$ values were determined to be $42.1 \pm 23.8 \mathrm{nM}$ and $1.4 \pm 6.7 \mu \mathrm{M}$, respectively.Figure 3.20 shows the raw and normalized binding curves for DZA3 and $\mathrm{AFB}_{1}$
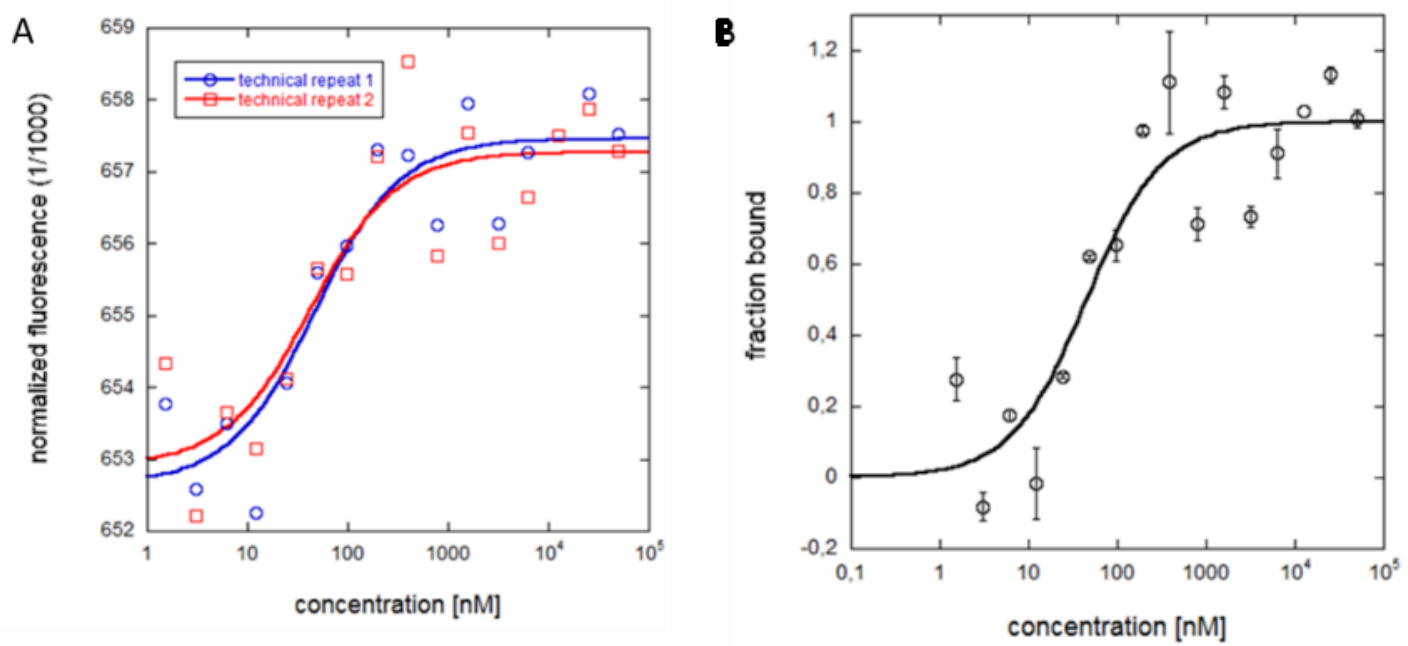

Figure 3.20: A) Binding curve for DZA3 and AFB1 B) Binding curve for DZA3 and AFB1 normalized to fraction bound.

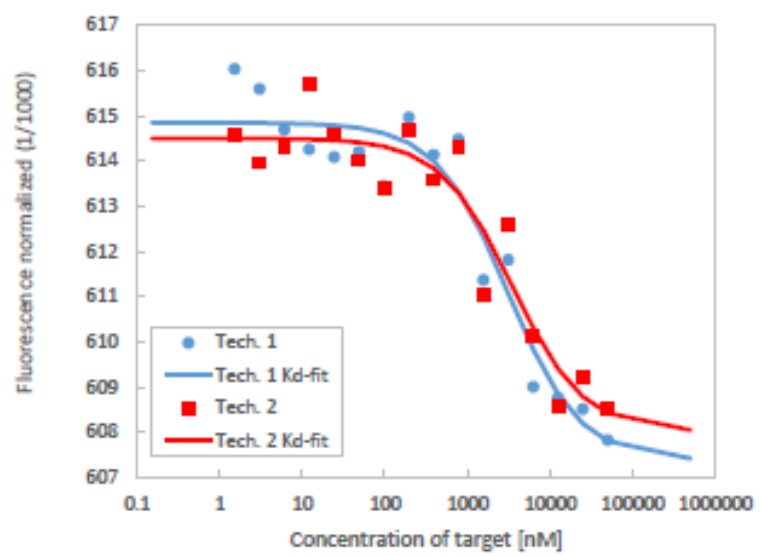

Figure 3.21: Binding curve from DZA7 challenged with $\mathrm{AFB}_{2}$

The six aptamer candidates were also tested against aflatoxins $B_{2}, G_{1}$, and $G_{2}$. It was found that DZA3 also bound $\mathrm{AFG}_{2}$ with high affinity. The reported $\mathrm{K}_{\mathrm{d}}$ from the MST was 
$15.3 \pm 4.8 \mathrm{nM}$. No measurable dissociation constants were determined for DZA5. DZA7, on the other hand, was determined to have a $\mathrm{K}_{\mathrm{d}}$ value of $3.3 \pm 0.2 \mu \mathrm{M}$ for aflatoxin $\mathrm{B}_{2}$.

The results of the MST studies that showed no measurable $\mathrm{K}_{\mathrm{d}}$ can be seen in Appendix C.
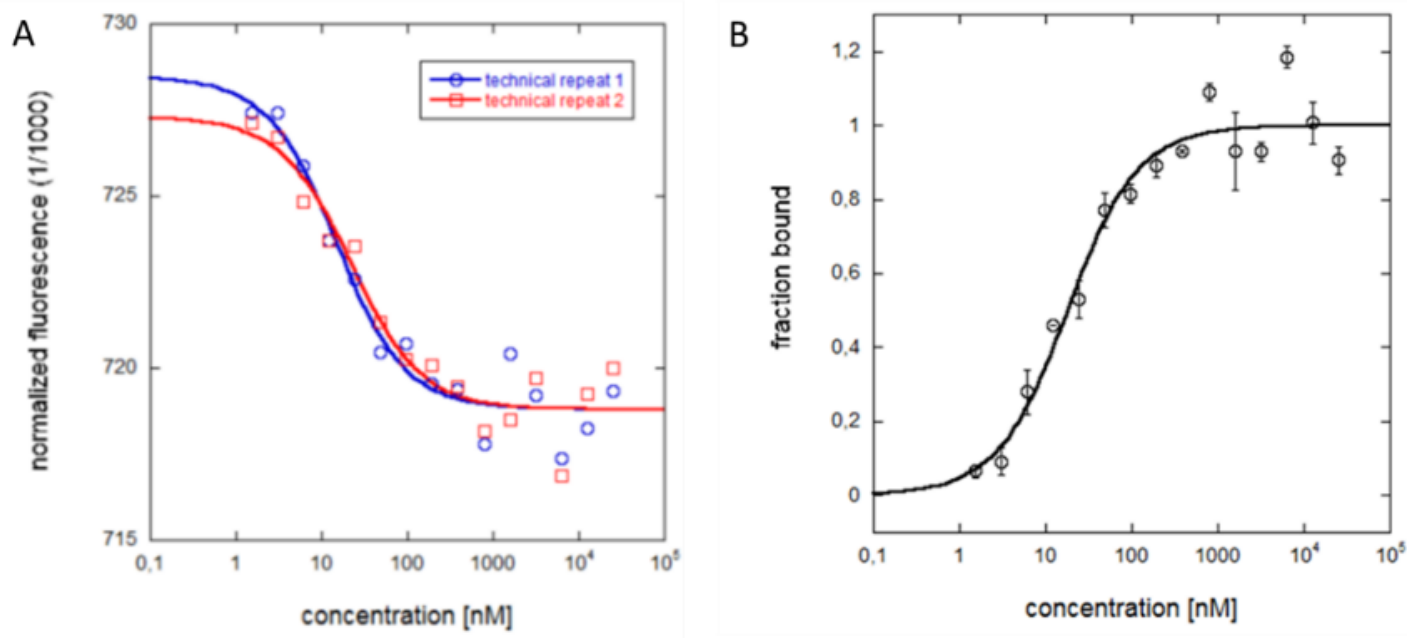

Figure 3.22: A) Binding curve for DZA3 and $\mathrm{AFG}_{2}$ B) Binding curve for $\mathrm{DZA3}$ and $\mathrm{AFG}_{2}$ normalized to fraction bound.
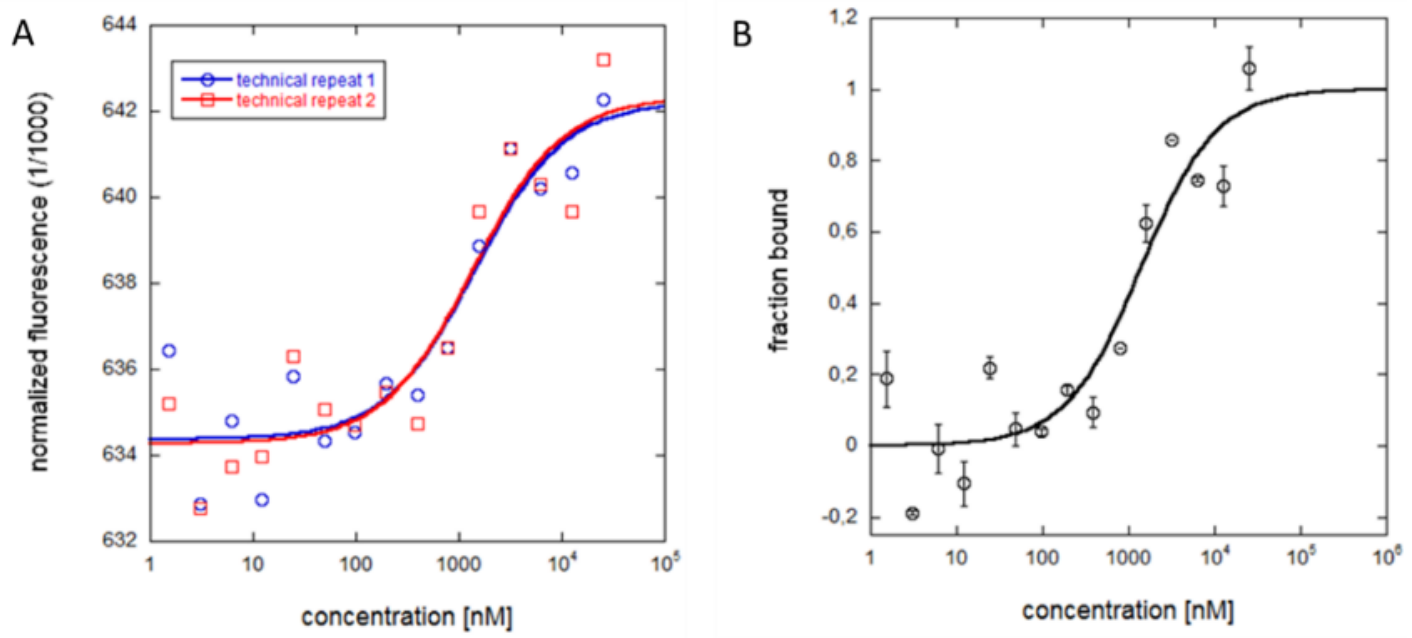

Figure 3.23: A) Binding curve for $3 M R 5$ and $A_{F B}$ B) Binding curve for $3 M R 5$ and AFB $_{1}$ normalized to fraction bound.

Of the random aptamers, $3 \mathrm{MR} 5$ was the only aptamer to exhibit a measurable $\mathrm{K}_{\mathrm{d}}$, with a value of $1.4 \pm 6.7 \mu \mathrm{M}$. 3MR5, 3MR3, and 3MR2 were also tested against $\mathrm{AFB}_{2}, \mathrm{AFG}_{1}$, 
and $\mathrm{AFG}_{2}$ but they were all determined to be nonbinding for the additional aflatoxins tested. The in-solution-based binding assay provides a suitable platform for analysing binding parameters for aptamers in conditions that are conducive to sensing applications. Moving forward MST will be a useful tool for obtaining conclusive evidence about the selectivity of the aptamers when challenged with counter-toxins.

\subsection{Conclusions}

The work highlighted in this chapter served to quickly screen aptamer candidates for aflatoxins using a multi-faceted approach. Of the original aptamer candidates, three sequences from each template design were selected for further characterization. Using structure characterization techniques such as recording the melting temperature profiles and circular dichroism, we maintain that the DZA aptamer candidates that were designed to contain structural biases show evidence of these structural features after the selection

process. Results also support that the previously performed SELEX successfully selected unmodified aptamers that display structure-switching abilities. Preliminary selectivity testing indicates that the aptamer candidates bind aflatoxin selectively. Cross-reactivity remains to be studied using microscale thermophoresis. Dissociation constants for the aptamer candidates were determined for individual aflatoxins.

Aptamers DZA3 and 3MR5 were shown to bind aflatoxin $B_{1}$ with high affinity. Their dissociation constants were determined to be $42.1 \pm 23.8 \mathrm{nM}$ and $1381.5 \pm 672.6 \mathrm{nM}$ respectively. Both aptamers showed consistent results for both the characterization 
studies and the binding assay. DZA3 and 3MR5 were therefore chosen as the optimal candidates for the development of structure-switching fluorescent biosensing platforms.

\section{Chapter: Nanoaptasensors for Aflatoxin}

\subsection{Statement of contribution}

Synthesis and purification of DZA3_BHQ1 were performed by Brandon Ho. Other syntheses were performed by Daniel Goudreau. Quenching measurements were performed by Daniel Goudreau and Brandon Ho.

\subsection{Introduction}

Molecular recognition is central to the regulated interactions of all biological process. Recognition events in proteins and nucleic acids elicit discrete structural changes. ${ }^{118,128}$ Synthetic recognition elements like their biological counterparts have been shown to elicit discrete, duplex-to-complex, structural changes in conformation when binding to their cognate ligands. ${ }^{57}$ Aptamers that are selected to bind small molecules have also been shown to undergo conformational changes. Methods that rely on structure-switching mechanism have been used to design aptamer-based colorimetric, electrochemical, and fluorometric sensors. ${ }^{117}$ Many aptamer-based detection methods mimic those that have been previously developed for antibodies. Those that do not require subsequent washing and separation steps have become progressively more

useful. ${ }^{74}$ Aptamers have shown great promise in fluorescence-based analytical applications due to their stability and ease of chemical modifications. 


\subsubsection{Incorporation of a single reporter}

Studies have shown that aptamers often undergo ligand-binding events that cause distinct changes in structural conformation. These distinct structural changes have been a driving force for biosensing. Fluorophores linked to a structurally labile region of an aptamer sequence can cause a change in the electronic environment of said Fluorophores. The change in environment is driven by the apparent "induced fit" conformational change of the aptamer binding its cognate ligand. ${ }^{80}$ Using fluorophore conjugated nucleobases in singly labelled sensors is common. In addition to these modifications, the use of fluorescent nucleotide analogues that are capable of pseudo base pairing such as 2aminopurine, and 3-methylisoxanthopterin have been reported. ${ }^{73}$ Changes in the local environment that result in the fluorescent quenching typically arise from base stacking interaction. Ligand-binding events causing a destabilization of duplexed regions within an aptamer result in a target dependent increase in fluorescence. A number of difficulties arise when dealing with the precise prediction of labile sequence regions. Also, it is difficult to predetermine whether sequences will undergo substantial conformational change, leading to a change in fluorescent properties of the fluorophore. Moreover, optimization steps for post-selection modified signalling aptamers is often a very lengthy process. ${ }^{57}$ Attempts have been made to circumvent these issues. Jhaveri et al. developed an in vitro selection method for fluorescently labelled signalling aptamers for ATP. The selection method involved a specialized pool of RNA aptamers that were skewed to 
contain fluorescein uridines. The method was the first of its kind to produce readily synthesized single reporter diagnostic reagents. ${ }^{74}$

\subsubsection{Incorporation of multiple reporters}

The simplest format for a FRET based assay is the incorporation of fluorophore and quencher or two compatible fluorophores in a single strand configuration. Förster resonance energy transfer (FRET) is a distance-dependent mechanism by which energy is transferred between two chromophores. Electronic excitation is transferred from an energetically excited fluorophore (donor) to a resonant state in another chromophore (acceptor) via coupling between two transition dipole moments. ${ }^{129}$ The resonance energy transfer is a non-radiative mechanism, as such, the donor returns to the ground state without undergoing fluorescence photon emission. This phenomenon is referred to as quenching and is observed via a decrease in donor fluorescence and a reduction of excited state lifetime. Based on the acceptor, an increase in acceptor fluorescence can also be observed. In order for FRET to occur between chromophores, there must be overlap between the fluorescence emission spectrum of the donor and the absorption spectrum of the acceptor. When considering FRET pairs, it was important to note that

increases in spectral overlap result in higher quenching efficiencies. ${ }^{130} \mathrm{FRET}$ is dependent on many factors including, but not limited to, the fluorescence quantum yield of the donor, and the spectral overlap of the two chromophores. Typically, efficient FRET can occur within the order of 10 to $100 \AA .^{131,132}$ 
Many homogeneous fluorescent sensing platforms employ FRET mechanisms. The incorporation of 3' and 5' terminal modifiers can be exploited for both the detection of FRET and its disruption. These designs function like molecular beacons and were first reported by Tyagi et al. Directly adjacent to the terminal modifiers is a 5-8 base complementary stem that dissociates during ligand binding. ${ }^{133}$ The disruption of FRET is utilized in "signal-on" strategies that are commonly engineered to induce spatial separation between the relative positions of the chromophores. Signal reduction schemes (turn-off) can also be utilized by hybridizing a partially complementary antisense strand to inhibit FRET. The use of commercially available quencher has allowed reductions in background fluorescence. This is especially true for the incorporation of dark quenchers such as the Black hole quencher family, and Iowa black quenchers. ${ }^{134}$ One method of doing this by sequestering the aptamer into to a nonbinding duplex state using a second sequence of partial complementarity. ${ }^{118}$

\subsubsection{Fluorescent hybridized probes}

Many studies have been devoted to the direct labelling of aptamers to optimize the performance and sensing ability of said aptamers. As briefly mentioned above, other methods that leverage the structure-switching properties for the sensing of targets involve the use of hybridized aptamer assemblies. ${ }^{108}$ DNA hybridization is a fundamental process in biology. The adaptation of strand-specific duplex formation through noncovalent bonds can be leveraged in sensing applications. ${ }^{135}$ Strand-specific duplex formation has been previously used for a wide range of techniques such as DNA architecture, DNAmediated organic synthesis, guided drug delivery, and nucleic acid-based biosensors. ${ }^{136-}$ 
${ }^{140}$ It is also useful in the design of structure-switching signalling aptamers. Decreasing the probability of secondary structure formation in the absence of target can significantly reduce the background allowing for lower signals to be measured reliably. ${ }^{73}$ Building on Jhaveri's work, Nutiu et al. (2005) later developed a selection protocol based on captureSELEX that could produce structure-switching signalling aptamers. In order to optimize the signalling properties of the aptamers, a duplexed structure utilizing a multi-reporter framework was developed. The developed signalling aptamers achieved limited quenching (4.5 fold) however, the aptamers were directly converted into their respective signalling probes. $^{57}$

It has been suggested that the observed affinity of a duplexed aptamer is both a function of the intrinsic affinity of the native aptamer and the hybridization free energy of the aptamer capture probe duplex. ${ }^{118}$ Modification to length location of antisense CPs as well as their length have been shown to affect observed affinity, and the performance hybridized biosensors. ${ }^{141,142}$ Porshetta et al. effectively tuned the relative affinity of cocaine hybridized signalling aptamers by over three orders of magnitude. As such, signalling performance can be greatly influenced by the design and optimization of signalling aptamers and is, therefore, a crucial step even in the selection and application of structure-switching signalling aptamers.

\subsubsection{Chapter objectives}

The aptamers in question were selected in a conformation-dependent manner. This chapter describes the use of the previously characterized aptamers in a fluorescent based sensing platform that exploits the aptamer's ability to adopt both a duplex structure 
and a complex structure when bound to the cognate ligand. The use of commercially available organic fluorophores was investigated in order to determine suitable modifiers in a multi-labelled FRET based system.

\subsection{Experimental}

\subsubsection{Materials}

Oligonucleotide sequences DZA3_Cy3,3MR_Cy3, 3MR_FAM, RCP_BHQ1, DZCP_TAM, RCP_TAM were purchased from Integrated DNA Technologies (Coralville, Iowa, USA). Aptamer sequences DZA3_FMA, and BHQ1 modified DZ capture probes were synthesized on a BioAutomation Mermade 6 oligonucleotide synthesizer. Phosphoramidites, sequence modifiers (BHQ1-dT, FAM-dT), activator, deblock, capping agents, and oxidizing agents were purchased from Glen Research. HPLC grade Acetonitrile was purchased from EMD Millipore. 1000 Å CPG columns were purchased from BioAutomation. Solutions were prepared with Milli-Q deionized water $\left(18.2 \mathrm{M} \Omega \cdot \mathrm{cm} @ 25^{\circ} \mathrm{C}\right)$. Buffering agents, salts, and detergents were purchased from Sigma-Aldrich. PAGE supplies were purchased from Bioshop Canada Inc. Aflatoxin $\mathrm{B}_{1}$ and $\mathrm{G}_{1}(1 \mathrm{mg})$ from Aspergillus flavus $\geq 98.00 \%$ (HPLC), and Aflatoxins $\mathrm{B}_{2}$ and $\mathrm{G}_{2}(1 \mathrm{mg}) \geq 98.00 \%$ (TLC) were purchased from Sigma-Aldrich and initially dissolved in $1 \mathrm{~mL} \mathrm{7:3} \mathrm{methanol-water.} \mathrm{Amicon-Ultra} 0.5 \mathrm{~mL} 3 \mathrm{kDa}$ centrifugal filters were purchased from Sigma-Aldrich. UV/Visible absorption spectra were acquired using a Varian Cary 300 Bio UV/Visible spectrophotometer. Fluorescence spectra were recorded using a Horiba Jobin Yvon Fluorolog spectrofluorometer. 


\subsubsection{DNA synthesis, purification and quantification}

Oligonucleotide sequences DZA3_FAM, and BHQ1 modified DZ capture probes were synthesized using standard phosphoramidite chemistry as previously described. 0.067 M Black Hole Quencher 1 (BHQ-1-dT) modifier was prepared with a 1:3 (v/v), anhydrous acetonitrile/dichloromethane diluent, and 0.067 M fluorescein-dT phosphoramidite solution was prepared with anhydrous acetonitrile.

\subsubsection{Glen-Pak ${ }^{\mathrm{TM}}$ cartridge purification}

FAM labelled sequences were purified by Glen-Pak. Six Glen-Pak purification cartridges were placed in the female luer ports of a 12 port SPE type vacuum manifold. The vacuum was adjusted to $\sim 10 \mathrm{~mm} \mathrm{Hg}$ using the vacuum control valve (targeted flow rate of 1 drop per second). Each cartridge was conditioned with $0.5 \mathrm{~mL}$ of acetonitrile followed by $1 \mathrm{~mL}$ of $2 \mathrm{M}$ tetraethylammonium acetate (TEAA).

Sequences that were purified using the Glen-Pak method were synthesized with DMT-ON. Deprotection of sequences was carried out by incubating the CPG beads in 1 $\mathrm{mL}$ of $28 \% \mathrm{NH}_{4} \mathrm{OH}$ for $17-24$ Hours. Once deprotected, an additional $1 \mathrm{~mL}$ of 100 $\mathrm{mg} / \mathrm{mL}$ sodium chloride solution was added to each microcentrifuge tube.

To purify the oligonucleotide sequences, the oligo/salt mixtures were added to the cartridges in $1 \mathrm{~mL}$ aliquots. $2 \times 1 \mathrm{~mL}$ aliquots of $100 \mathrm{mg} / \mathrm{mL} \mathrm{NaCl}$ salt wash solution was used to wash the failed sequences from the cartridges. Cartridges were then rinsed with 2 x $1 \mathrm{~mL}$ of $2 \%$ trifluoroacetic acid (TFA), and $2 \times 1 \mathrm{~mL}$ of deionized water. Clean $15 \mathrm{~mL}$ microcentrifuge tubes were placed in the manifold and the purified DNA sequences were 
eluted from the cartridge using $2 \times 1 \mathrm{~mL}$ of $50 \%$ acetonitrile in deionized water containing $0.5 \%$ ammonium hydroxide. Eluted samples were collected and dried overnight on cryopump. Oligonucleotide sequences were confirmed using mass spectrometry.

\subsubsection{Glen-Pak ${ }^{\mathrm{TM}}$ desalting of oligonucleotides}

Cartridges were prepared as previously described for oligo cartridge purification. PAGE purified oligonucleotides samples were made up to $2 \mathrm{~mL}$ in $0.1 \mathrm{M}$ TEAA. Solutions were loaded into cartridges in $1 \mathrm{~mL}$ aliquots. Cartridges were then flushed with a minimum of $2 \mathrm{~mL}$ of deionized water. Elution of desalted oligonucleotide was performed with $2 \times 1 \mathrm{~mL}$ of $50 \%$ acetonitrile/deionized water containing $0.5 \%$ ammonium hydroxide. Eluted were collected and dried overnight on cryopump. Oligonucleotide sequences were resuspended in buffer and quantified using optical absorbance at $260 \mathrm{~nm}$. Molar extinction coefficients were determined using the nearest neighbour approximation (IDT Oligoanalyzer 3.1) as previously described.

\subsubsection{Page Purification}

The BHQ1 modified oligonucleotide sequences were then purified using 12\% denaturing polyacrylamide gel electrophoresis (PAGE) using the same method as describe in Section 2.3.2.1. Purified DNA was quantified by measuring the optical absorbance at $260 \mathrm{~nm}$. Molar extinction coefficients were determined using the nearest 
neighbour approximation (IDT oligoanalyzer 3.1), taking in to account the $\varepsilon 260 \mathrm{~s}$ of the modifiers used. Oligonucleotide sequences were confirmed using mass spectrometry.

\subsubsection{Quenching Measurements}

Various concentrations of complementary $\mathrm{CP}$ sequences were optimized against a fixed concentration of fluorescently labelled aptamer i.e. $100 \mathrm{nM}$. For each fluorophore/quencher pair, the molar ratios of the aptamer to complementary strand that were investigated were set at 1:1,1:2, 1:3,1:4 and 1:5. QE samples were made up to 100 $\mu \mathrm{L}$ with Mg SELEX buffer. DNA samples were annealed by first heating the mixture to $90^{\circ} \mathrm{C}$ for 15 minutes on a dry heat block. The samples were then allowed to cool to room temperature for an additional 30 minutes, and stored at $4^{\circ} \mathrm{C}$ for 24 hours. Samples were allowed to warm to room temperature for $30 \mathrm{~min}$ prior to use. The fluorescence measurements were recorded using a $60 \mu \mathrm{L}$ quarts cuvette on a Horiba Jobin Yvon Fluorolog spectrofluorometer. An excitation wavelength of $495 \mathrm{~nm}$ was used for fluorescein labelled DNA. Emission was recorded from 500-600 nm in $1 \mathrm{~nm}$ increments and an integration time of $0.5 \mathrm{~s}$. A $555 \mathrm{~nm}$ excitation wavelength was used for Cy3 labelled DNA. Emission spectra were recorded from $565-650 \mathrm{~nm}$ in $1 \mathrm{~nm}$ increments.

\subsubsection{Structure switching aptamer assay}

For the quenching measurements, varying concentration of the $\mathrm{CP}$ was hybridized with a fixed concentration of aptamer. $100 \mathrm{nM}$ of fluorescently labelled aptamer was combined with $1 \mathrm{x}$ to $5 \mathrm{x} \mathrm{CP}$ concentrations and vortexed. Samples first annealed using the standard hybridization method and stored at $4^{\circ} \mathrm{C}$ for 24 hours prior to use. In order to 
avoid time dependent changes in fluorescence, each sample was kept at $4^{\circ} \mathrm{C}$ until used. Sample containing only singly labelled DNA was prepared in a comparable manner. The DNA was not heated as part of the annealing process. Once made, it was stored a $4^{\circ} \mathrm{C}$ with the annealed samples. The samples were allowed to warm to room temperature for 30 min before use. Each sample was challenged with $1 \mu \mathrm{M}$ aflatoxin $\mathrm{B}_{1, \text { vortexed and }}$ allowed to incubate for 1 hour. Samples were made up a total volume of $100 \mu \mathrm{L}$ with $\mathrm{Mg}$ SELEX buffer. The fluorescence measurements were recorded using a $60 \mu \mathrm{L}$ quartz cuvette on a Horiba Jobin Yvon Fluorolog spectrofluorometer with a SpectrAq controller. An excitation wavelength of $495 \mathrm{~nm}$ was used for fluorescein labelled DNA. Emission was recorded from 500-600 nm in $1 \mathrm{~nm}$ increments and an integration time of $0.5 \mathrm{~s}$. A $555 \mathrm{~nm}$ excitation wavelength was used for Cy3 labelled DNA. Emission spectra were recorded from $565-650 \mathrm{~nm}$ in $1 \mathrm{~nm}$ increments

\subsection{Results and Discussion}

\subsubsection{Choosing FRET pairs}

The incorporation of commercially available dye phosphoramidites are extremely convenient and often times cost-effective. ${ }^{143}$ Although, not all dyes can be acquired as phosphoramidites. Fluorescein (FAM) was used as a fluorescent reporter during some of the characterization experiments. Keeping with this, a quencher that showed considerable spectral overlap with FAM's emission spectrum was chosen $\left(\lambda_{\mathrm{ex}}=495 \mathrm{~nm}, \lambda_{\mathrm{em}}=520\right.$ $\mathrm{nm}$ ). Black hole quencher (BHQ1) is known as a dark quencher; a chromophore that returns to ground state energy in a non-radiative fashion. It and other dark quenchers have become useful acceptors for FRET since they have little to no intrinsic fluorescence. 
They can, therefore, be used in high concentrations without contributing background fluorescence within the donor-emission channel. ${ }^{134}$ Many dark quenchers are commercially available, covering absorption maxima from 453 to $672 \mathrm{~nm}$. BHQ1 ( $\lambda_{\mathrm{ex}}=$ $534 \mathrm{~nm}$ ) was used as the primary quencher due to its availability as a deoxythymidine derivative. Another fluorophore commonly used to label nucleic acids, that was investigated was Cy3. Cy3 $\left(\lambda_{\mathrm{ex}}=555 \mathrm{~nm}, \lambda_{\mathrm{em}}=568 \mathrm{~nm}\right)$ was used in association with BHQ1 as they have considerable spectral overlap. A third alternative was explored. FAM and TAMRA $\left(\lambda_{\mathrm{ex}}=558 \mathrm{~nm}\right)$ were previously the gold standard for dual label FRETbased assays. However, TAMRA is not a dark quencher and has its own emission band at $577 \mathrm{~nm}$ that can contribute to background fluorescence in the donor-emission channel.

\subsubsection{Design of signalling probes}

The strategy utilized for the selection of aptamers DZA3 and 3MR5 consisted of a fixed central domain that was incorporated into the aptamers. This fixed central domain that was complementary to a (5') biotinylated capture probe allowed the aptamer to be immobilized to a solid support via hybridization. This strategy takes advantage of the aptamer's inherent duplex-to-complex structure switching ability, and in turn was the focus of developing simple solution-based signalling platform. To coordinate the structure-switching process with a measurable signal, the sequence and complementary element $(\mathrm{CP})$ were modified with a fluorophore and quencher. The signalling method for the hybridized signalling probe is therefore based on a conformationally dependent fluorescence-dequenching mechanism. ${ }^{144}$ 
Convention typically places the quencher moieties on the 3'-terminus and the fluorophore on the 5 '-terminus. ${ }^{145}$ This, however, is not necessarily the case when designing hybridized signalling probes. Since the characteristic aptamer design utilized a fixed central domain for immobilization purposes, it was presumed that the latent central domain was not essential to the ligand-binding region. Based on this assumption, modifications were made within or directly flanking the central aptamer domain. Due to remaining FAM labelled capture probe from the characterization experiments, initial experiments were performed with a fluorophore labelled DZCP_FAM and a BHQ1 modified DZA3 aptamer. The desired sites chosen for modification were determined based on several factors. Firstly, altering the sequence to accommodate the modifiers was avoided if possible. Many of the internal sequence modifiers were obtained as thymidine phosphoramidites. The FAM dye that was used as an internal modifier were derivatized to a deoxythymidine nucleoside through a C6 linker. Similarly, BHQ1 the primary quencher used was acquired as deoxythymidine phosphoramidite $\mathrm{Cy} 3$ in particular was not obtained as a dT derivative. Cy3 was doubly linked to the DNA backbone via two short C3 linkers on each of Cy3's nitrogen atoms. The designated modification site is therefore abasic (see Figure 4.1). In the case of 6carboxyfluorescein labelled capture probes, a 5'-fluorescein modifier was used. Table 4.1 to Table 4.4 show the aptamer sequences used and the determined location for each of their modifications. Modifiers were positioned in order for FRET to occur $(10-100 \AA)$. 


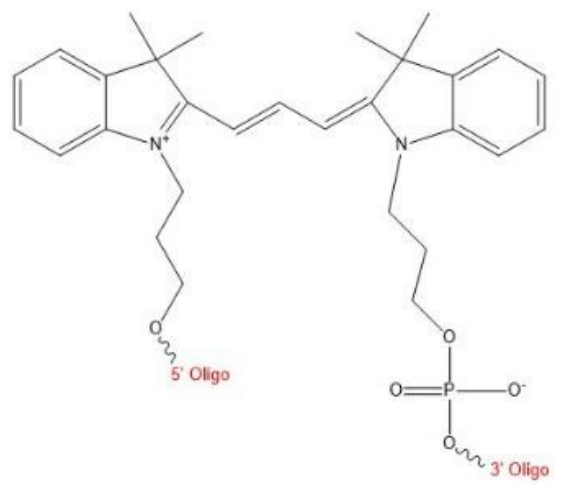

Figure 4.1: Chemical structure of dually-linked Cyanine 3 modifier

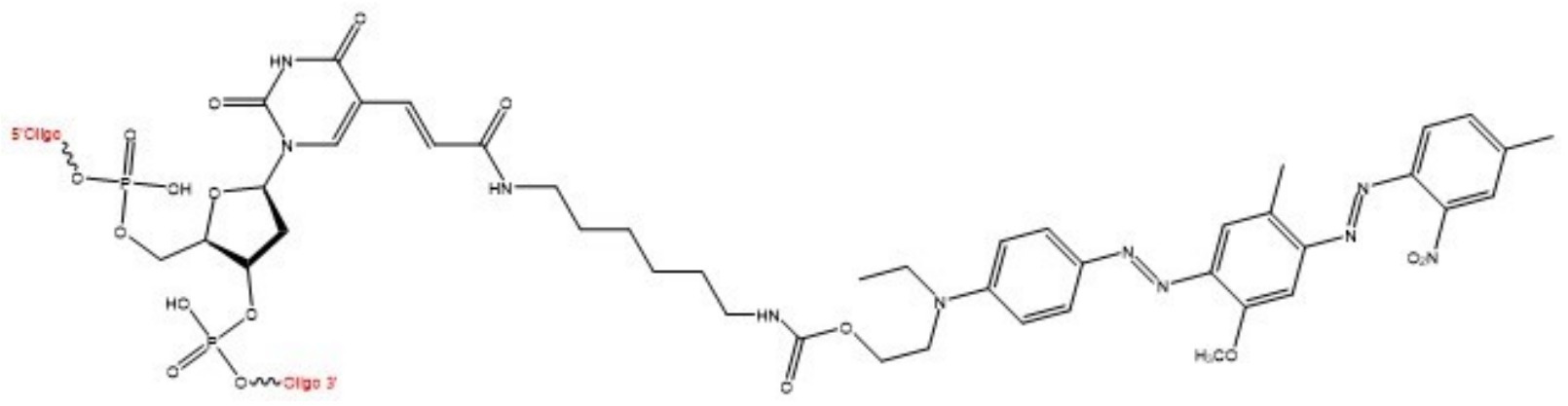

Figure 4.2: Chemical structure of Black hole quencher 1 deoxythymidine modifier (BHQ1-dT)

Table 4.1: Modified DZA3 aptamers used for the design of signalling probes

\begin{tabular}{ll}
\hline Name & Sequence (5' to $\left.\mathbf{3}^{\prime}\right)^{*}$ \\
\hline \multirow{2}{*}{ DZA3_FAM } & AGCAGCACAGAGGTCAGATGGCATGACAAGTAAAGTTTTGTGGGFA \\
& GGGCGGGTTGGTTTTGTTGACAGATGTATCCCTATGCGTGCTACCGTG \\
\hline \multirow{2}{*}{ AA } & AGCAGCACAGAGGTCAGATGGCATGACAAGTAAAGTTTTGBGGGTA \\
& GGGCGGGTTGGTTTTGTTGACAGATGTATCCCTATGCGTGCTACCGT \\
& GAA \\
DZA3_Cy3 & AGGAGCACAGAGGTCAGATGGCATGACAAGTAAAGYTTTTGTGGGT \\
& TGAA \\
\hline
\end{tabular}

*F = Fluorescein label, B = BHQ1 label, $\mathrm{Y}=\mathrm{Cy3}$ label 
Table 4.2: Modified 3MR sequences used for the design of hybridized signalling probes

\begin{tabular}{|c|c|}
\hline Name & Sequence $\left(5^{\prime}\right.$ to $\left.3^{\prime}\right) *$ \\
\hline \multirow{3}{*}{ 3MR5_FAM } & CAGCACAGAGGTCAGATGTGGCGCCTCACACGTGCAATCGAAFGAC \\
\hline & TTCGTTCGAGGCTCATAATCGTCCTCGGTCTATGATGTATCCCTATG \\
\hline & $\overline{\text { CGTGCTA }}$ \\
\hline \multirow{3}{*}{ 3MR5_Cy3 } & CAGCACAGAGGTCAGATGTGGCGCCTCACACGTGCAAYCGAATGAC \\
\hline & TTCGTTCGAGGCTCATAATCGTCCTCGGTCTATGATGTATCCCTATG \\
\hline & $\overline{\mathrm{CGTGCTA}}$ \\
\hline
\end{tabular}

*F = Fluorescein label, $\mathrm{Y}=\mathrm{Cy3}$ label

Table 4.3: Modified DZA capture probes used for the design of hybridized signalling probes

\begin{tabular}{ll}
\hline Name & Sequence (5' to 3')* \\
\hline DZCP_FAM & TTTTTTTTTTAACCCGCCCTACF \\
\hline DZCPnT_FAM & AAC CCG CCC TAC F \\
\hline DZCPM1_FAM & AAC CCG CAC TAC F \\
\hline DZCP9.0_FAM & CCG CCC TAC F \\
\hline DZCP_BHQ1 & TTTTTTTTTTTAACCCGCCCBAC \\
\hline DZCPnT_BHQ1 & AACCCGCCCBAC \\
\hline DZCPm1_BHQ1 & AACCCGCACBAC \\
\hline DZCP9.0_BHQ1 & CCGCCCBAC \\
\hline DZCPnT_TAM & AACCCGCCCRAC \\
\hline *F $=$ Fluorescein label, $\mathbf{B}=\mathbf{B H Q 1}$ label, $\mathbf{R}=\mathbf{T A M R A}$ label
\end{tabular}

Table 4.4: Modified 3MR capture probes used for the design of hybridized signalling probes

\begin{tabular}{ll}
\hline Name & Sequence (5' to 3')* \\
\hline RCPnT_BHQ1 & GCCTCGAACGAAB \\
\hline RCPnT_TAM & GCCTCGAACGAAR \\
\hline *B = BHQ1 label, R = TAMRA label
\end{tabular}

\subsubsection{Quenching Measurements}

In order to test the feasibility of the hybridized signalling probe, it was important to demonstrate the quenching efficiencies that were attainable using various FRET pairs. In order to do so, various concentrations of the complementary capture probe were tested against a fixed concentration of aptamer $(100 \mathrm{nM})$. The initial testing was performed using remaining DZCP_FAM and a newly synthesized BHQ1 modified DZA3. The molar ratios of DZCP_FAM to DZA3_BHQ1 that were investigated were 1:0.5, 1:1, 1:2, 
1:3, 1:4, and 1:5. Hybridization of the two complementary sequences provides a spatial separation of roughly $10.2 \AA$ along the duplex. Additional separation is allowed by the modifier's flexible C6 linkers. For each test, the aptamer and capture probe was annealed following a standard hybridization protocol, by which the DNA was heated to $90^{\circ} \mathrm{C}$ to disrupt intramolecular interactions. The DNA was then allowed to cool to room temperature gradually and subsequently cooled to $4^{\circ} \mathrm{C}$ for short-term storage. Quenching measurements were determined using spectrofluorimetry. The quenching efficiency $(\mathrm{QE})$ was taken as the difference in fluorescence (\%) of the hybridized assembly, as compared to the fluorescently labelled capture probe of the same concentration. Initial testing with DZA3_BHQ1/DZCP_FAM showed significant decreases in quenching at low aptamer/CP ratios. The 0.5:1 ratio demonstrated a QE of $92 \%$. The 1:1 ratio also showed significant decreases in fluorescence. The QE was determined to be $45.6 \%$. Higher capture probe ratios showed very little quenching due to the large excess in the fluorescently labelled complementary strand. Higher ratios also resulted in a much greater variance in fluorescence measurements. 


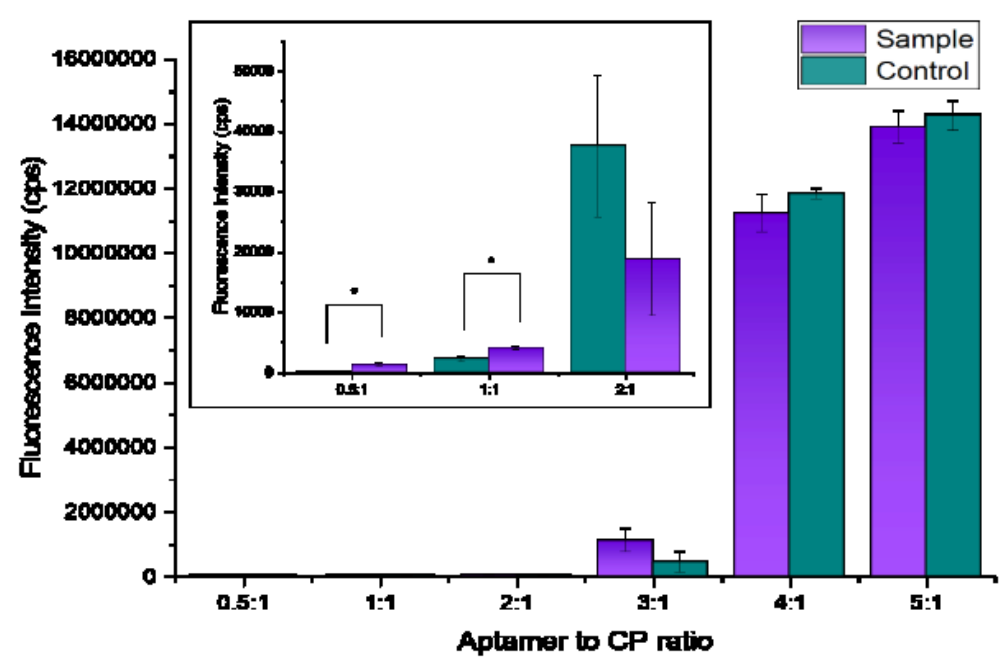

Figure 4.3: Quenching measurements for fixed DZA3_BHQ1 concentration in combination with increasing 24-nt DZCP_FAM sequence.

The reporter arrangement for the two DNA strands showed some limitations. Firstly, higher ratios of complementary strand would cause a very large background fluorescence, which is disadvantageous. Using excess short capture sequences has been reported to drive higher hybridization efficiencies as well as decrease background fluorescence. Hybridization ratios have been proposed between 1:1.5 to 1:11 for similar structure-switching assays. ${ }^{59,139,146}$ It is also important to note that when looking at the $0.5: 1$ ratio, it is only useful in determining the upper limits of quenching. Due to the reporter arrangement, the $0.5: 1$ ratio puts the aptamer in excess of the $\mathrm{CP}$ making it less than ideal for sensing purposes. It was therefore decided that given the high background 
fluorescence at concentrations that could benefit the degree of hybridization, the reporters would be moved to opposite strands.

\section{DZA3 FAM/BHQ 1 Combination}

DZA3_FAM was the first aptamer to be tested. At this point, minor modifications were made to the capture probes to determine if small changes in complementarity would affect the complexation of the two strands and cause a change in fluorescence quenching. Three capture altered capture probes were investigated. The original CP contained a 12base long T-tail that was originally biotinylated for the purpose of immobilizing the $\mathrm{CP}$ during SELEX. The first alteration that was made was truncating the T-tail. The resulting CPnT was a 12-nt, fully complementary CP. Two shorter capture probes were also investigated, 12-nt sequences that contained a base mismatch central to the sequence, and a 9-nt CP that was further truncated (see Table 4.3).
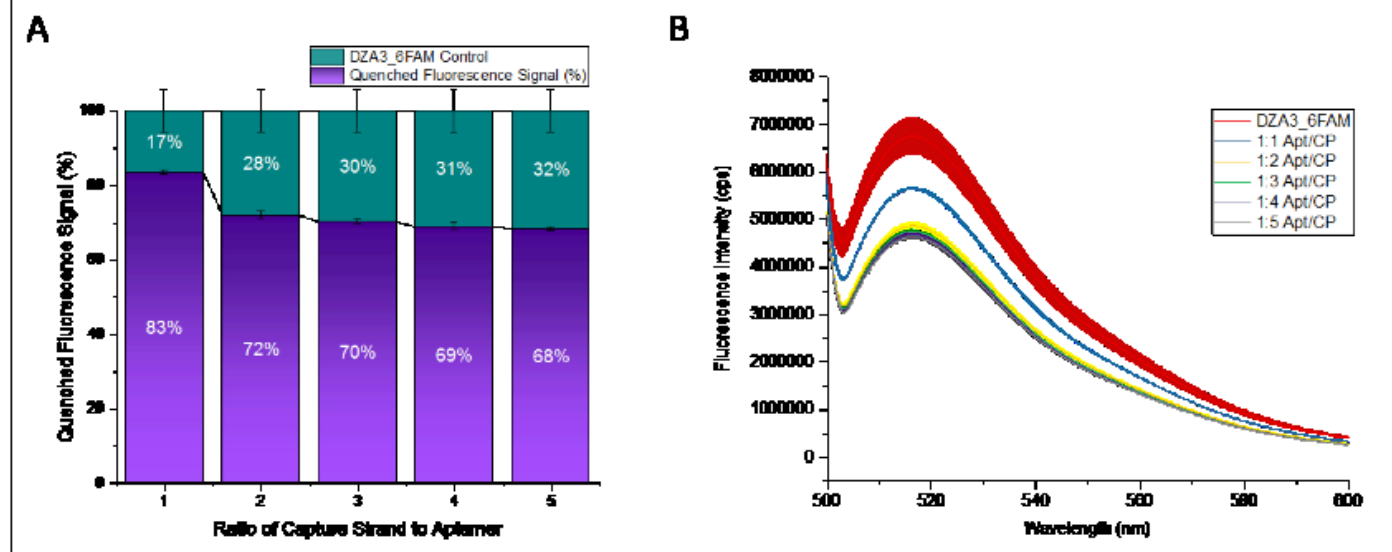

Figure 4.4: A) Quenching measurements for varying aptamer capture probe ratios using DZA3_FAM and 24-nt DZCP_BHQ1 sequences. B) spectral data resulting from quenching efficiency experiments. The error bars are represented as shadows of the same colour underlying each line in the spectrum. 


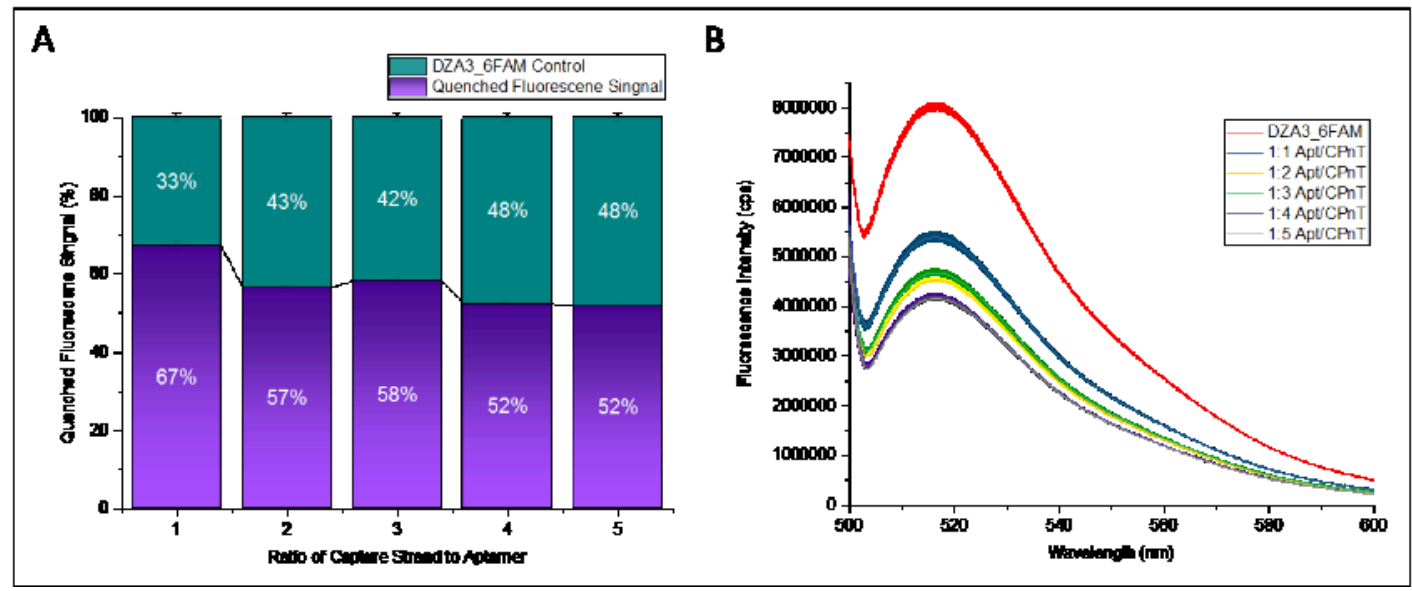

Figure 4.5: A) Quenching measurements for varying aptamer capture probe ratios using DZA3_FAM and 12-nt DZCPnT_BHQ1 sequences. B) spectral data resulting from quenching efficiency experiments. The error bars are represented as shadows of the same colour underlying each line in the spectrum.

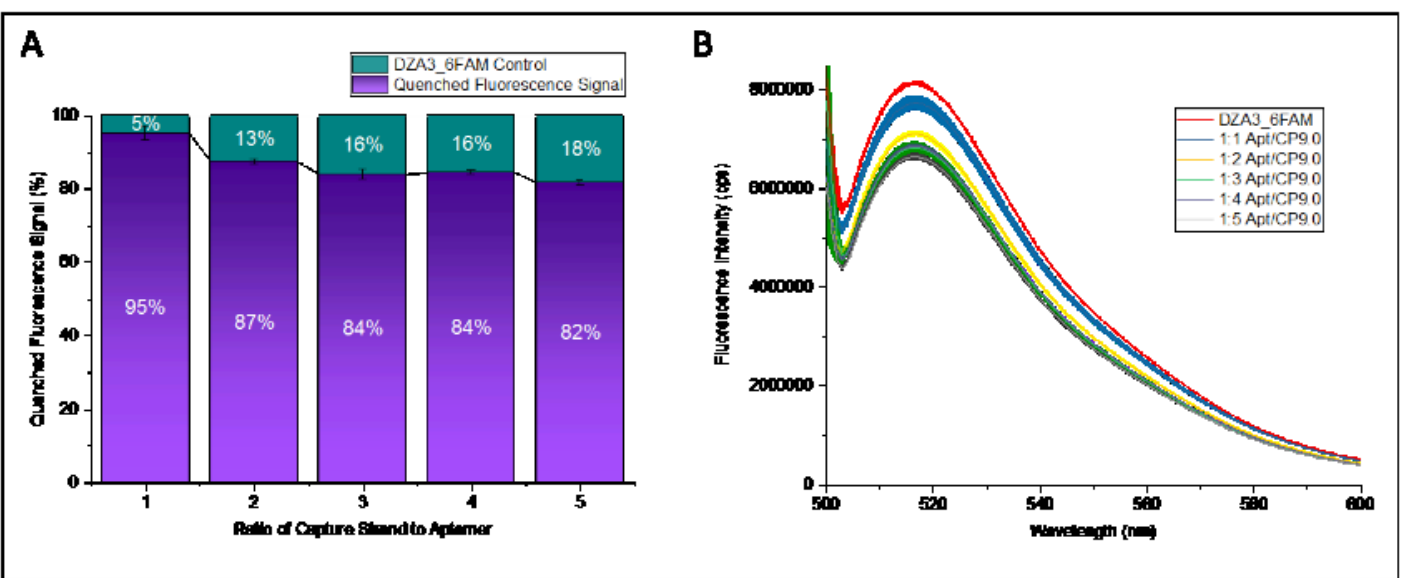

Figure 4.6: A) Quenching measurements for varying aptamer capture probe ratios using DZA3_FAM and 9-nt DZCP9.0_BHQ1 sequences. B) spectral data resulting from quenching efficiency experiments. The error bars are represented as shadows of the same colour underlying each line in the spectrum. 


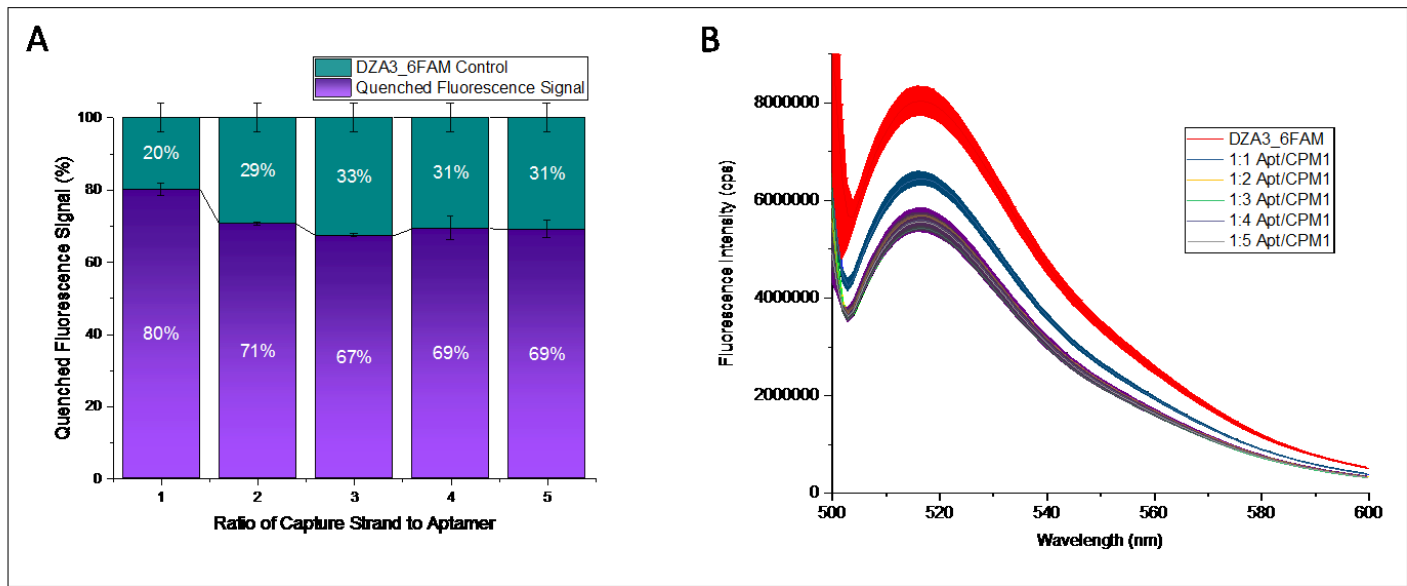

Figure 4.7: A) Quenching measurements for varying aptamer capture probe ratios using DZA3_FAM and 12-nt DZCPM1_BHQ1 sequences. B) spectral data resulting from quenching efficiency experiments. The error bars are represented as shadows of the same colour underlying each line in the spectrum.

DZA3_FAM and its multiple CPs showed varying quenching efficiencies. In the majority of experiments, the quenching efficiencies would plateau at ratios of 1:3 and above. Figure 4.4 to Figure 4.7 show the quenching efficiencies for the four CPs tested with DZA3_FAM. DZCPnT_BHQ1 and DZCP_BHQ1 differ significantly with $~ 50 \%$ and $\sim 30 \%$ QE respectively. This is likely caused the by a destabilizing effect that the long overhang has on the capture probe. As expected, the QEs showed decreasing QE values with decreased complementarity, with DZCP9.0 being the lowest at under $20 \%$ QE. These results may become more important when further exploring the ligand mediated release of the capture probe in the presence of target. Based on the determined QEs it was concluded that the 12-base pair forming DZCPnT_BHQ1 hybridized with the 
highest affinity. As a result, the remaining FRET pairs were investigated using the 12-nt capture probes with the truncated T-tails.

\section{DZA3_Cy3/BHQ 1 Combination}

$\mathrm{Cy} 3$ and BHQ1 were the second FRET pair to be investigated. Cyanine dyes are commonly used fluorescent labels. They are characterized by a polymethine bridge linking two nitrogens with a delocalized charge. The fluorescent properties of the dyes can be changed by controlling the length of the polymethine bridge. The name $\mathrm{Cy} 3$ originates from its three carbon polymethine bridge. As previously stated, Cy3 is an intense orange-fluorescent label with an emission maximum at $568 \mathrm{~nm}$. Contrary to the other dyes used, $\mathrm{Cy} 3$ is not attached through a flexible carbon linker. Cy3 is doubly linked to the DNA backbone. The position of the fluorescent label was directly flanking the central domain of the DZA3 aptamer as to not hinder the formation of the Gquadruplex structure. The hybridization of the two sequences provides roughly $34 \AA$ of separation.

Of the FRET pairs tested for the DZA3 aptamer, the Cy3/BHQ1 combination had one of the highest $\mathrm{QE}$ values at high aptamer to capture strand ratios $(65 \%)$. It was also observed that the QE values did not plateau at higher ratios like the FAM labelled aptamer. The high QE values may be a product of the restriction in dye orientation due to the double phosphoramidite linkage, providing less variability in dye orientation and potentially resulting in static dye-dye interaction. 


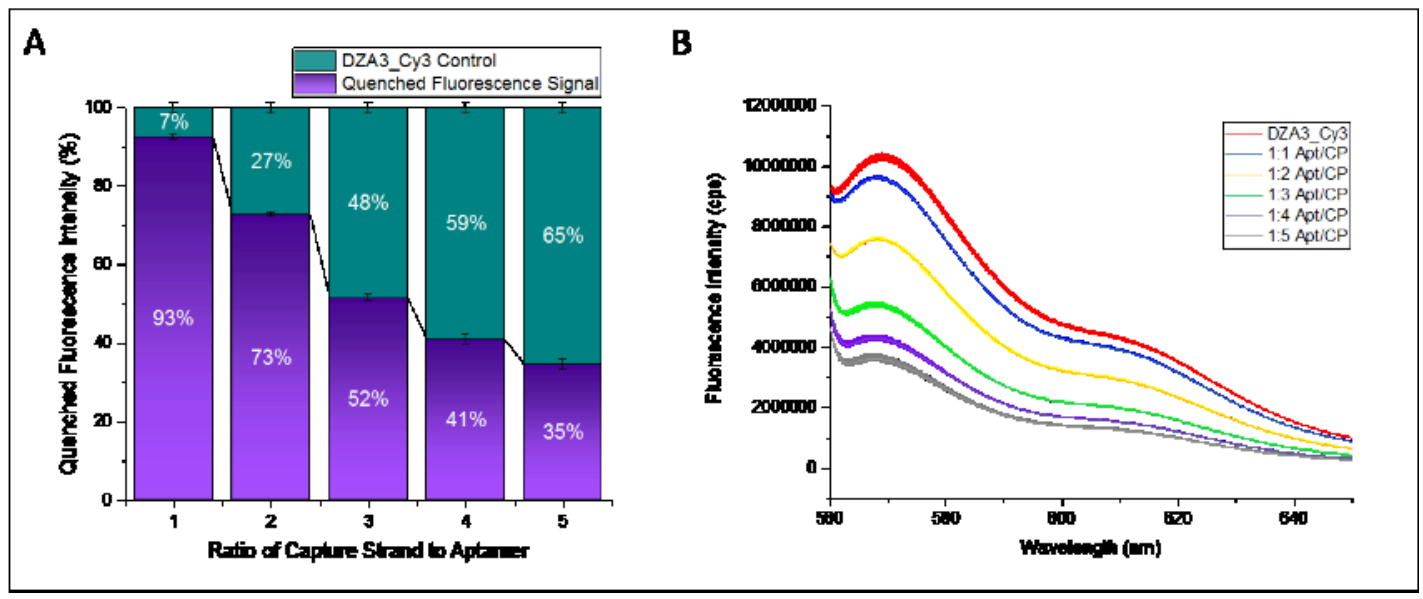

Figure 4.8: A) Quenching measurements for varying aptamer capture probe ratios using DZA3_Cy3 and 12-nt DZCPnT_BHQ1 sequences. B) spectral data resulting from quenching efficiency experiments. The error bars are represented as shadows of the same colour underlying each line in the spectrum.

\section{MR5_FAM/BHQ1 Combination}

Fluorescein was also used as a fluorescent label on the 3MR5 aptamer. The 12-nt "no tail" RCPnT was used as the antisense strand. This sequence was labelled with BHQ1 at the 3' terminus. The carboxy fluorescein-dT modification places the two chromophores $27.2 \AA$ apart along the duplexed strands.

3MR5 showed impressive quenching efficiencies. At a 1:1 ratio, the 3MR5_FAM/RCP_BHQ1 had a QE of $65 \%$. The highest obtained QE was $88 \%$, which was obtained with $3 x$ quencher strand. This level of quenching was consistent from 1:3 to 1:5. Though the 3MR candidates have shown what appears to be more efficient hybridization for their antisense strand, QE values may differ based on modifier position along the strand. Internal modifiers were positioned in a way that did not alter the aptamer sequence. As such, they were not all positioned within the same distance. In comparison to the DZA3_FAM combination, chromophores are separated by an 
additional $\sim 20 \AA$. This is not considering the length provided by the C6 linkers. The proximity of the two modifiers in DZA3_FAM could affect the relative dye orientation. The effectiveness of the hybridization could also be influenced by steric effects based on the size of the chromophores.

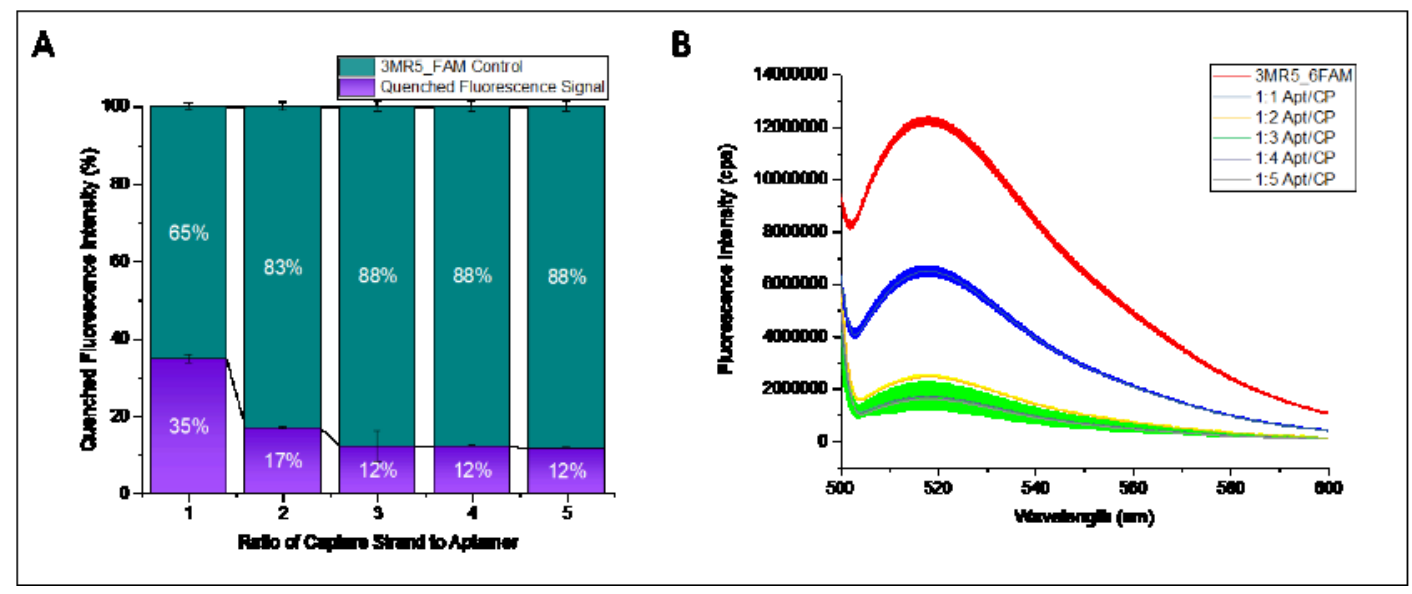

Figure 4.9: A) Quenching measurements for varying aptamer capture probe ratios using 3MR5_FAM and 12-nt RCPnT_BHQ1 sequences. B) spectral data resulting from quenching efficiency experiments. The error bars are represented as shadows of the same colour underlying each line in the spectrum.

\section{MR5_Cy3/BHQ1 Combination}

Of the 3MR aptamer candidates, 3MR5 had the highest binding affinity for aflatoxin $\mathrm{B}_{1}\left(\mathrm{~K}_{\mathrm{d}}=1.4 \pm 6.7 \mu \mathrm{M}\right)$. While 3MR5 did not have a $\mathrm{K}_{\mathrm{d}}$ in the low nanomolar range like DZA3, it was important to investigate its viability as a hybridized signalling probe. Throughout the aptamer characterization experiments, it was noted that the random candidates appeared to bind their CPs with higher affinity. This was attributed to the DZA aptamer's higher propensity for forming higher order secondary G-quadruplex structures. Based on the location of the modifiers, there was a separation of roughly 
$30.5 \AA$ along the DNA strands. The flexible C6 linker affords more flexibility and slightly more distance for the BHQ1 modifier.

Figure 4.10 shows the $\mathrm{QE}$ results and the spectral data from the 3MR5_Cy3/RCP_BHQ1 combination. This signalling probe demonstrated higher QE values than those seen in with the DZA aptamer. The highest $\mathrm{QE}$ recorded at the 1:5 ratio was roughly $80 \%$. The leveling-off of $\mathrm{QE}$ values at higher aptamer/CP ratios that was present in previous combinations were observed for 3MR5_Cy3/RCP_BHQ1. A small change in profile for the fluorescence emission spectra of the 3MR5_Cy3 was also observed by means of a flattening of sorts without substantial decreases in fluorescence intensity.

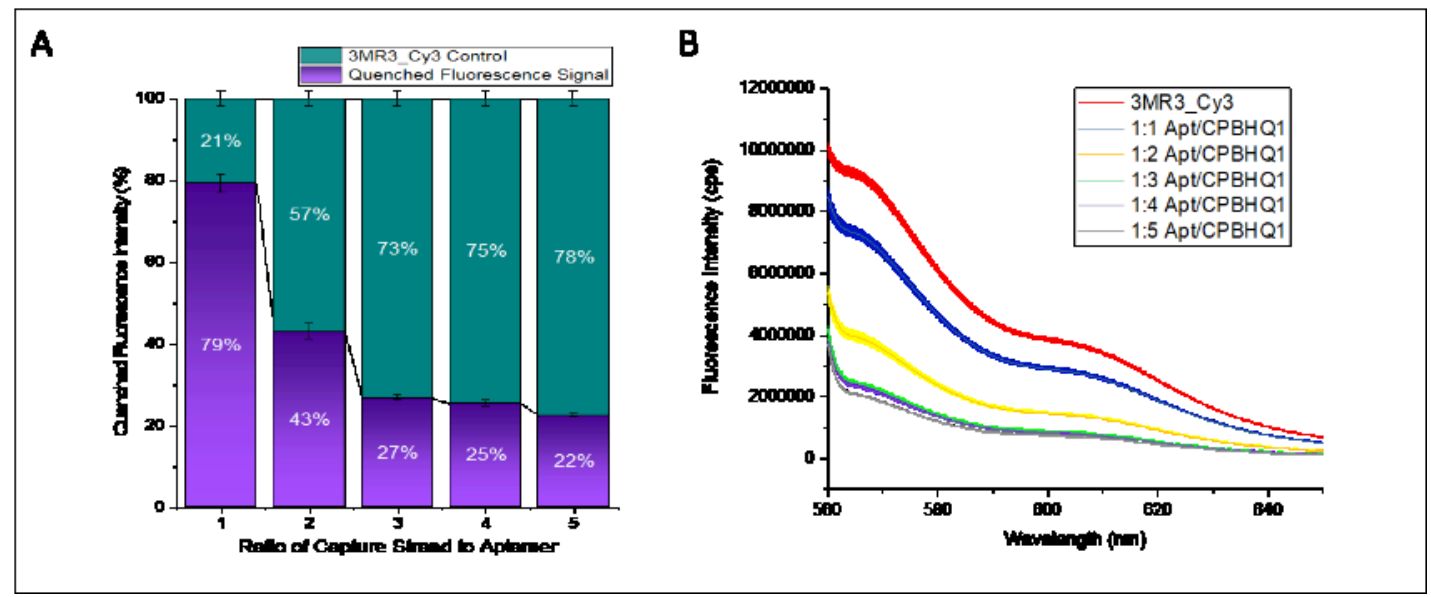

Figure 4.10: A) Quenching measurements for varying aptamer capture probe ratios using 3MR5_Cy3 and 12-nt RCPnT_BHQ1 sequences. B) spectral data resulting from quenching efficiency experiments. The error bars are represented as shadows of the same colour underlying each line in the spectrum.

Cy3 and BHQ1 are known to be very efficient FRET pairs. The donor and acceptor structures play a role in dynamic quenching and what is known as static quenching. 
Static quenching involves the formation of a non-fluorescent ground state complex or a heterodimer. The static quenching for a fluorophore-quencher pair is more efficient that FRET and is not necessarily reliant on spectral overlap. Aggregation of dyes in aqueous solvents is controlled by electrostatic, steric, and hydrophobic forces. ${ }^{147}$ Cyanine and rhodamine dyes are planar, rather hydrophobic, and have delocalized charge due to the quaternary nitrogens. ${ }^{143} \mathrm{FAM} / \mathrm{BHQ} 1$ could potentially result in static quenching as well still, it is not as likely as Cy3/BHQ1. Dye-dye interactions could potentially be studied through their absorption spectrum if the heterodimers formed produced distinct absorption profiles. Future studies could also look at fluorescent lifetime measurements in order to determine if static quenching is occurring within the hybridized probes. The QE results of the Cy3/BHQ1 combinations were different when comparing the DZA3 and 3MR5 aptamers. Early, it was suggested that DZA3 had a higher propensity for forming complex structure and therefore could kick off the CP more readily. It should also be noted that DZA3 has almost a 10\% percent higher guanine content than 3MR5. These guanine bases are primarily focused in the G-rich central domain which is directly flanked by the modifier positions. The placement of a reporter in relation to proximal nucleotide composition can yield fluorescence quenching based on inherent base-specific quenching. It is recommended that fluorophores should not be placed directly next to Grich sequences. ${ }^{148}$

TAMRA combinations were also investigated however the QE values obtained were the lowest of all the combinations tested. As expected, the TAMRA labelled probes did show higher levels of background fluorescence. In the goal of maximizing both quenching efficiency and fluorescence recovery of the hybridized signalling probe with 
the target, the FAM/TAMRA signalling probes were not further investigated as a potential FRET pairs.

\subsubsection{Structure switching signalling duplex}

The primary goal of determining efficient FRET pairs for the labelling of the hybridized signalling probes is to optimize them for ligand mediated release of the antisense capture strand. Figure 4.11 provides a schematic representation of the sensing platform. When hybridized, the aptamer is intentionally inhibited. Upon binding of the cognate ligand, the aptamer undergoes a structural change that activates the fluorescence dequenching, such that the binding event has an observable signal.

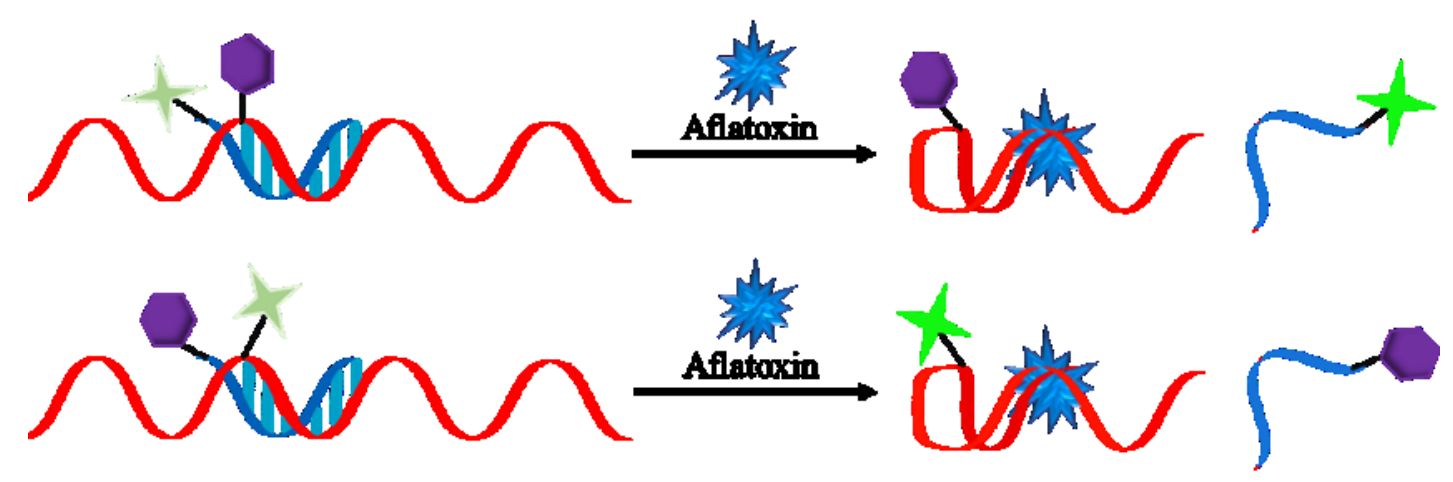

Figure 4.11: Schematic representation of structure-switching hybridized signalling platform for the detection of AF. Inversely modified FRET assembly (Above) Common arrangement for FRET assembly (Below).

In order to demonstrate the concept of the duplex to ligand-complex transition, the hybridized signalling probes were initially challenged with a single target concentration. The SELEX experiments were performed using a total mixture of aflatoxins. Initially, the goal was to produce an aptamer that could bind the four major types of aflatoxins based 
on the underlying structural commonalities of the aflatoxin analogues. The results of the MST studies indicated that only one of the aptamers, DZA3, produced low $\mathrm{K}_{\mathrm{d}}$ values for more than one analogue. Both DZA3 and 3MR5 showed a high binding affinity for $\mathrm{AFB}_{1}$. As such, when testing the ligand-induced dequenching of the hybridized complexes the aptamers from each candidate group were only challenged with the $\mathrm{AFB}_{1}$ target.

As a consequence of the chromophore arrangement in the first assembly (DZA3_BHQ1/DZCP_FAM), quenching measurements indicated high fluorescence background at high $\mathrm{CP}$ ratios. This effect was to be expected. As previously stated, concentrations of aptamer/complementary sequence in biosensor platforms have been reported from 1:1 to 1:11. This is done to ensure proper hybridization while decreasing overll background fluorescence. As seen in Figure 4.3, High quenching efficiencies were only obtainable at low aptamer/CP ratios. The initial tests with DZA3_BHQ1/DZCP_FAM were carried out with an aptamer/CP ratio of 1:1. Additionally, each of the inversely modified probes was tested using the modified CPs.

Table 4.5 summarizes the modified CPs used, their complementarity, and the results of the ligand mediated dequenching.

Table 4.5: Summary of the fluorescence recovery obtained when challenging structure-switching signalling probes with $10 \mathrm{nM}$ aflatoxin $\mathrm{B}_{1}$

\begin{tabular}{ccccc}
$\begin{array}{c}\text { Sequence } \\
\text { name }\end{array}$ & Sequence (5'-3') & $\begin{array}{c}\text { Base } \\
\text { pairs }\end{array}$ & $\begin{array}{c}\text { Quenching } \\
\text { Efficiency (\%) }\end{array}$ & $\begin{array}{c}\text { Fluorescence } \\
\text { Recovery (\%) }\end{array}$ \\
\hline DZCP & TTT TTT TTT TTT AAC CCG CCC & 12 & $63.9 \pm 2.3$ & - \\
DZCPnT & TAC F & 12 & $71.0 \pm 3.1$ & $9.1 \pm 0.4$ \\
DZCPM1 & AAC CCG CCC TAC F & 9 & $44.8 \pm 4.2$ & $16.4 \pm 6.1$ \\
DZCP9.0 & CCG CCC TAC F & 9 & $36.3 \pm 4.1$ & $26.5 \pm 7.8$ \\
\hline
\end{tabular}


The inversely modified aptamer assemblies produced results that appeared to be greatly influenced by the complementarity of the aptamer and capture probe. DZCPnT showed the highest quenching efficiency of the CPs tested, but the lowest fluorescence recovery when challenged with target. Oppositely, DZACP9.0, which had the lowest complementarity, showed the lowest quenching efficiency, but the highest fluorescence recovery. The preliminary signalling probe tests were also carried out by incubating the assemblies with OTA and SB. Neither of the controls exhibited significant increases in fluorescence. While the results of the initial testing with the inverse capture probes seemed promising, the alternative arrangement was investigated in order to circumvent the inherent limitations that the inversely modified probe demonstrated. As previously stated for the quenching measurements, the results of the modified CP studies prompted the use of the 12-nt truncated CPnTs for the remainder of the probe combinations. The first quenching experiment to provide good quenching efficiencies was the DZA3_FAM/CPnT_BHQ1 combination. From these results, the hybridized assembly was tested against aflatoxin at a single concentration. The two sequences were hybridized in a 1:1.2 in order slightly improve the hybridization efficiency. The quenching efficiency fell between the previously tested 1:1 and 1:2 QE results $(\sim 38 \%)$ The aptamer was challenged with an equimolar concentration of $\mathrm{AFB}_{1}$ in the goal of producing a measurable increase in fluorescence. 


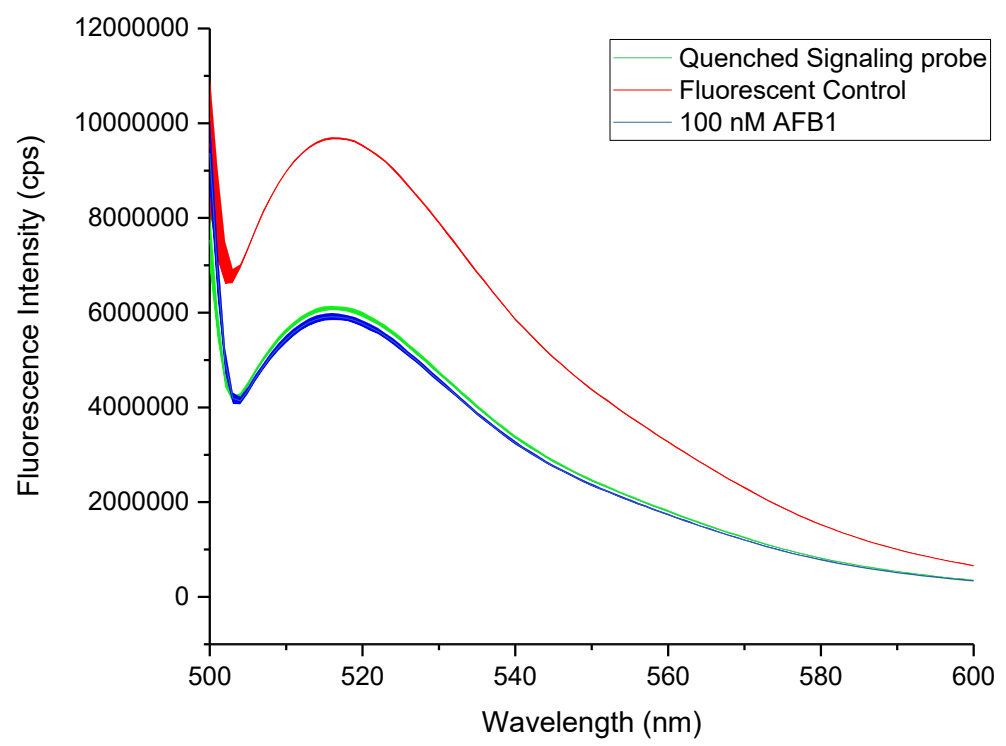

Figure 4.12: DZA3_FAM/DZCPnT_BHQ1 quenching (37.3\%) and testing with $100 \mathrm{nM}$ AFB1. Challenging hybridized probe with equimolar target showed no significant increase in fluorescence.

Figure 4.12 shows the quenched aptamer assembly after being incubated with aflatoxin. There appeared to be no significant increase in fluorescence. From this, it was decided that all subsequence QE experiments be tested with aflatoxin. The concentration to be tested was increased 10-fold in order to determine whether or not a large excess of target would cause the expected increase in fluorescence.

During the quenching efficiency experiments, each of the aptamer/capture probe ratios was tested against a single $\mathrm{AFB}_{1}$ concentration. Contrary to what was expected, the dequenching of the hybridized probes did not occur. With the addition of $\mathrm{AFB}_{1}$, the observed fluorescence for top FRET combinations all showed decreased responses. 
Appendix E contains examples of the spectral fluorescence that demonstrates the decreases that resulted from the remaining hybridized signalling probe combinations of interest. As was seen in the QE measurements, maximal quenching is achieved with combinations of aflatoxin and high $\mathrm{CP}$ ratios. The effect of aflatoxin is more notable at lower CP ratios. For example, Appendix E.2 shows an additional 40\% quenching when the 1:1 DZA3_Cy3/BHQ1 combination is incubated with $1 \mu \mathrm{M}$ aflatoxin. The results may suggest that the presence of target does not effectively displace the capture probe since the samples are quenched further. FRET techniques generally are more efficient than techniques that rely on nucleobase-specific quenching of fluorophores. Effective displacement of the $\mathrm{CP}$ would likely result in an increase in fluorescence even if the fluorescence intensity is delimited by the structurally induced quenching of the dyes. This series of results prompted the investigation of the influence of aflatoxin $\mathrm{B}_{1}$ on each of the fluorescently labelled aptamers.

\subsubsection{Sequence effect on fluorescein}

The fluorescein in DZA3_FAM is directly flanking the G-rich central domain of the aptamer and is positioned at the end of a three-guanosine tract.

DZA3_FAM alone showed a decrease in fluorescence of $13.6 \%$ with the addition of $\mathrm{AFB}_{1}$. Nucleobase-specific quenching has been reported for a number of dyes including fluorescein, rhodamine, oxazine, and coumarin dyes among others. ${ }^{149}$ Photoinduced electron transfer plays a role in nucleobase-specific quenching. Adjacent guanosines in particular exhibit fluorescence quenching due to their low oxidation potential. ${ }^{148}$ Moreover, the high density of guanosines in G-quadruplexes have been utilized in 
quencher-free molecular beacons that primarily utilize fluorescein as a fluorophore. ${ }^{150,151}$ The drop in fluorescence intensity suggests that the fluorescein reporter is affected by the proximal guanosines that are present in the central domain. Furthermore, the possible presence of a G-quadruplex structure could have a similar effect. This would also suggest that the formation of the G-quadruplex structure occurs when the aptamer-target complex forms. It is unlikely that $\mathrm{AFB}_{1}$ itself is directly causing the decrease in fluorescence intensity. $\mathrm{AFB}_{1}$ is named for its blue fluorescence. $\mathrm{AFB}_{1}$ has an excitation maximum near $365 \mathrm{~nm}$, and therefore should not contribute to quenching of the fluorescent label. ${ }^{152}$ Another possible explanation for the unpredicted quenching is the effect of proximal guanosines in ssDNA in comparison to duplex DNA. Nazarenko et al. report the interaction of guanosine in ssDNA producing up to a 2 -fold decrease in fluorescence. Upon duplex formation, the quenching ability of the proximal guanines was eliminated. ${ }^{153}$ The secondary structure predictions for DZA3 and 3MR5 show high degrees of stem-loop, and hairpin formation, particularly in the portions of the sequences that were altered with the fluorescein dye. Based on the length of the sequences it is very much possible that the structural changes that occur upon complex formation include the disassembly of pre-existing duplexed regions and the formation of new ones. Based on this the results would then support that in its native form, the fluorescein modifier is part of a duplexed portion of the sequence. 

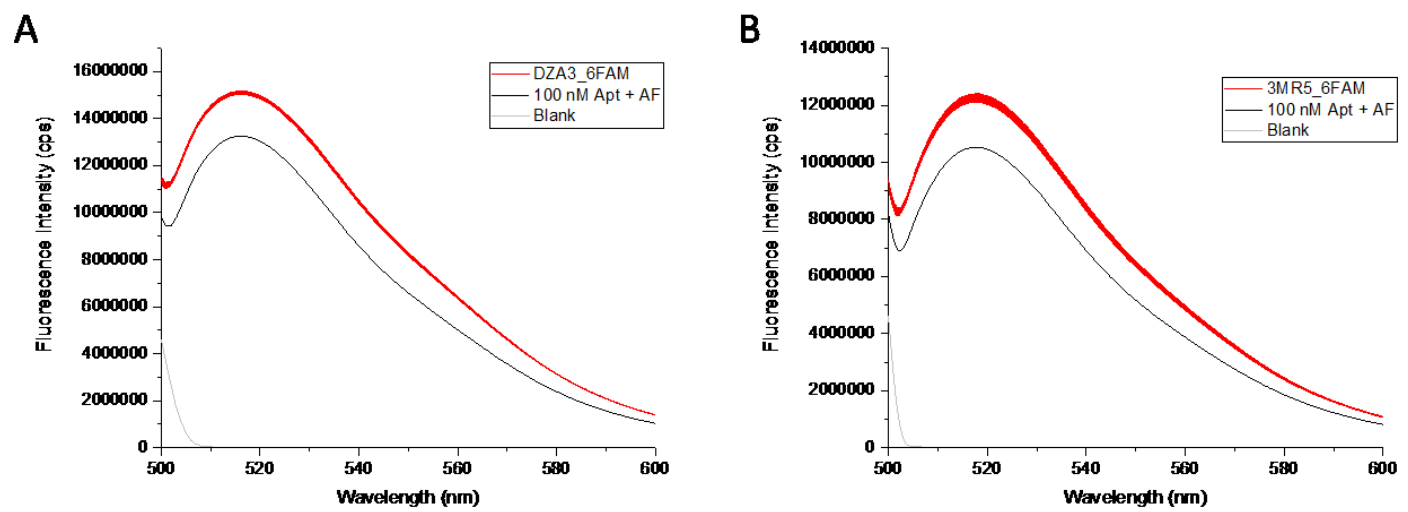

Figure 4.13: Structure dependent fluorescence intensity decrease with the addition of 10x excess aflatoxin $B_{1}$ A) DZA3_FAM resulted in a 13.6\% decrease in fluorescence B) 3MR5_FAM resulted in a $14 \%$ decrease in fluorescence.

The notion that the G-rich central domain of DZA3_FAM was responsible for the formation of a quenching of G-quadruplex structure was not supported by the results of the preliminary 3MR5_FAM test. 3MR5_FAM showed a decrease in fluorescence intensity of $14.0 \%$ with the addition 10 -fold excess of $\mathrm{AFB}_{1}$. This quenching is within the same range as that observed for DZA3_FAM, in the presence of the same amount of target. Contrary to the DZA sequence, the fluorescein-dT modifier is situated next to a single guanosine. The two aptamers are different in terms of sequence composition. Two similarities do arise, however. The first, as previously stated, is the immediate neighbouring of at least a single guanosine. The second is the rather long length of the aptamers. The DZA aptamer being 97-nt, and the 3MR aptamer being 100-nt in length. The similar quenching supports the ability for guanosine to quench fluorescence based on H-bonding and whether it is duplexed or not. This also suggests that the fluorescence the of labelled DNA is altered based on their secondary structures making it a useful tool in its self. 


\subsubsection{Sequence effect on $\mathrm{Cy3}$}

As seen in the preliminary results for the singly labelled fluorescein aptamers, the testing with Cy3 also produced decreases in fluorescence. The observed effect of secondary structure on the fluorescence intensity was greater. DZA3_Cy3 had a 42.2\%

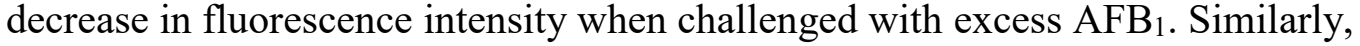
3MR5_Cy5 was $44.5 \%$ percent quenched with the addition of $\mathrm{AFB}_{1}$.

Cyanine dyes are some of the most popular fluorophores for biophysical applications because they exhibit high photostability. Alternatively, cyanine dye fluorescence is strongly sequence-dependent. Experiments have shown that cyanine dye fluorescence is affected by local environment. Most work has studied the effects of proximal nucleobases in terminally labelled cyanine, and the effect of base-stacking with terminal base pairs. $^{154,155}$

A

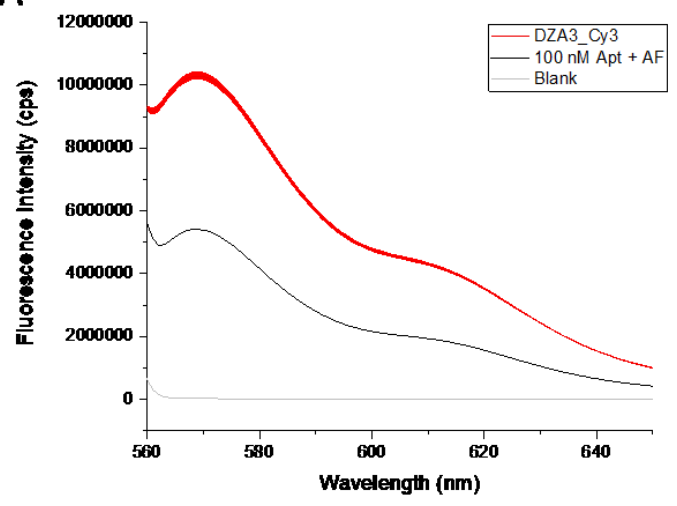

B

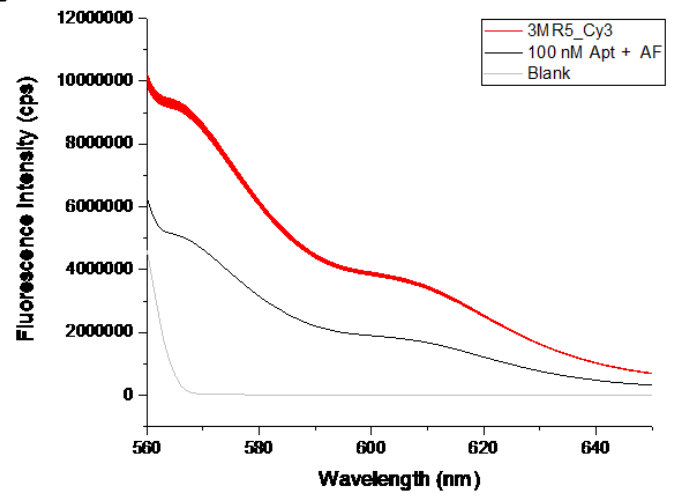

Figure 4.14: Structure dependent fluorescence intensity decrease with the addition of 10x excess aflatoxin $B_{1}$ A) DZA3_Cy3 resulted in a 42.2\% decrease in fluorescence B) 3MR5_Cy3 resulted in a $44.5 \%$ decrease in fluorescence 
The cyanine modifier used for the DZA and 3MR aptamers was not available as a deoxythymidine derivative. The internal Cy3 modification is doubly linked to the DNA backbone via short $\mathrm{C} 3$ linkers. Fluorescein, for instance, has a flexible 6-carbon linker. The relative orientation, location, and interactions between nucleotides and the dyes are dynamic and seldom known. In contrast to other fluorophores, the doubly linked Cy3 is expected to be rotationally constrained. Cy3 can also undergo a deactivation mechanism which has long been studied. The capacity to undergo photo-induced isomerization of the excited singlet state can be restricted in sterically constrained environments. ${ }^{156,157}$ Herein, it is suggested that the singly modified Cy3 aptamers adopt some form of stable secondary structure that alters the photophysical properties of the dye. This results in a rotationally constrained, and uninhibited cyanine dye. High fluorescence efficiencies as a result of the interaction with DNA reduces the efficiency of trans $\rightarrow$ cis isomerization from the singlet excited state. Alternatively, in the presence of $\mathrm{AFB}_{1}$, the observed intensity is reduced significantly. This supports the notion that the structural changes that occur upon complexation with the ligand result in reduced sequence rigidity within that portion of the sequence. Following the photoinduced isomerization, an efficient nonradiative process results in a deactivated ground-state cis photoisomer causing a decrease in intensity. Given that the modifiers are arranged in what is predicted to be a structurally labile non-binding portion of the aptamer, the resulting structural changes could very well cause the lower degrees of secondary structure in proximity to the aptamer's central domain. Both sequences showed very similar quenching for the same fluorophore regardless of their nucleobase composition. The photophysical changes in fluorophore characteristics are a consequence of changes in local environment. This effect, however, 
appears to be caused by the structural changes that occur within the binding and nonbinding regions of both aptamers, and less so from the nucleobase-specific environment. As previously discussed, the Ellington group reported the screening analysis of aptamer for the contribution of the constant sequence regions. It was concluded that the constant regions were not prominent in overall binding aptamer binding regions. ${ }^{158}$

Changes in the fluorescence intensity based on sequence specific interaction with a singly labelled aptamer may present certain limitations. Fluorescence can be modulated by a series of dynamic interactions between the dye and building blocks of the aptamer. This can result in measured fluorescence intensities that are disproportional to the amount of labelled aptamer and target in solution. In order to determine whether a singly labelled aptamer which depends on structural rearrangement to produce a measurable signal is a viable option, further experimentation is needed. Stennett et al. state that the use of fluorescent-based techniques for a variety of purposes has far surpassed the required physicochemical studies that are crucial to further understanding effects of local environment on specific fluorophores. ${ }^{157}$ Reports also indicate that little work has been performed with elongated sequences comparable to those in this study. Based on current findings it is suggested that an assembly which contains a fluorophore labelled aptamer of this length would undergo sequence secondary structure dependent changes in fluorescence, thereby altering the measured FRET dequenching signal.

One way of circumventing this issue would be to design a tripartite duplex signalling probe. The chromophores are arranged on two separate halves of an antisense stand. Hybridization of the three DNA component duplex would bring the acceptor and donor in close enough proximity for efficient FRET to occur. The aptamer, in this case, 
would not be modified therefore, ligand binding would induce a complete disassembly of the hybridized probe would avoid any structurally induced changes in fluorescence. Similar designs have been presented for the incorporation of structure-switching aptamers for sensing applications. ${ }^{144}$

\subsection{Conclusions}

The goal of this chapter was to utilize a hybridized signalling probe designed to take advantage of the duplexed inhibited state in a multi-reporter FRET system. The inversely modified hybridized probes demonstrated fluorescence signal recovery when tested with aflatoxin. When testing with alternate CPs, it was determined that lower complementarity and the destabilisation caused by a single mismatch resulted in lower $\mathrm{QE}$, but higher fluorescence recovery. The opposite was true for CPs with higher complementarity. The hybridization of signalling probes is often performed at high ratios to increase hybridization efficiency while decreasing fluorescent background. When dealing with the reverse signalling probe, it was not possible to do so. While the fluorescence recovery did show some promise, the focus was placed on a signalling probe that contained the fluorophore on the aptamer and a quencher on the antisense strand (CP). This approach is more generic than the one initially investigated.

The investigation of alternative chromophore arrangements and FRET pairs was also examined at this point. From the quenching ratios, it was determined that the addition of a large excess of $\mathrm{AFB}_{1}$ caused further quenching of the hybridized assemblies. The results from this chapter provide evidence that both aptamers interact with their linked fluorophores in a way that influences the measured fluorescence signal. It was posited 
that the structural changes of DZA and 3MR aptamers influence the fluorescence of the dyes in a similar fashion and that FAM and $\mathrm{Cy} 3$ are quenched through different mechanisms. Given the length of the sequences, and the fact that the CPs are hybridized a non-binding portion of the sequence it could also be suggested that the aptamer binding of aflatoxin does not disrupt the duplexed portion. Knowing the limitations that arise from modifying such a long structure-switching aptamer with a fluorophore. It would be beneficial to further investigate assemblies in which the aptamer is not directly linked to the fluorophore. One way of doing so would be to adopt a tripartite signalling probe, also referred to as a binary probe, in which the acceptor and acceptor are linked to two separate halves of an antisense strand. Through little modification, it would be possible to further investigate FRET pairs with the inversely modified probes. An alternative FRET pair may provide sufficient quenching at low aptamer/CP ratios. Additionally, the ligand-induced disassembly could avoid unwanted interaction with the aptamer.

\section{Chapter: Contributions to knowledge and future studies}

Mycotoxins are natural contaminants that affect a number of food commodities. Aflatoxins are primarily of concern in crops that grow in tropical and subtropical climates. They are potent carcinogens and have been linked to high a prevalence of liver cancer in certain regions of the world. ${ }^{159}$ Managing mycotoxin exposure to mitigate health risks is therefore of utmost importance. Moreover, there is a need for detection methods whose primary attributes are their ease of use and cost effectiveness. The use of high throughput screening methods prior to confirmatory analysis has been shown to significantly decrease the cost of mycotoxin analysis. ${ }^{25}$ Aptamers provide a stable and 
cost-effective alternative for high-affinity recognition elements in several biosensor platforms. Their chemical synthesis and in vitro selection result in their ease of modification as well as their use in non-physiological conditions.

Current confirmatory analytical methods for mycotoxin detection require sophisticated infrastructure, equipment, supplies, and qualified technicians. As such, there is a growing need for rapid and cost-effective screening methods that do not necessitate tedious pre-treatment or require skilled technicians. High sensitivity is imperative due to low permissible contamination levels established by several regulatory bodies. Typically, limits for aflatoxins range from 0.05 to $20 \mathrm{ppb}$ depending on the food commodity. Current analytical approaches used by regulatory bodies that are less reliant on infrastructure include TLC, and immunological methods. TLC has been a reliable method used for the analysis of mycotoxins for over 50 years. ${ }^{160}$ Their most significant drawbacks are their need for stable supplies of solvents and standards, and their safe storage ${ }^{160}$ On the other hand, immunological methods are typically provided as test kits with all necessary standards and solvents. While immunological methods typically have limited shelf-life and require refrigeration, the incorporation of test kits, sampling equipment and training models for use in mycotoxin endemic regions are the most viable option for analysis. As such, aptamer-based sensing platforms that are intended for use in regions which are limited by their lack of political commitment and infrastructure must be easily incorporated into testing kits that do not require sophisticated instrumentation. ${ }^{160}$ Examples of alternative methods for aflatoxin detection that could be suitable for use in both rural areas as well as the management the of contaminated bulk commodities have included the use of microfluidic chips able to detect aflatoxin $\mathrm{B}_{1}$ at 
concentrations as low as $0.25 \mathrm{ppb} .{ }^{79}$ More closely related to the work presented in this document, Sharma et al. developed a structure switching signaling aptamer assay for the detection of $\mathrm{AFM}_{1}$ in milk that was able to detect aflatoxin concentrations as low as 25 ng/Kg. ${ }^{139}$ Additionally, aptamer-based assays such as these can have the added benefits of decreased matrix effect, and crossreactivity. The development of additional sensing platforms for multiple aflatoxin contaminants that benefit from low production costs and portability could potentially rival existing immunological methods that are currently accepted by regulatory bodies around the World.

The above research presented the characterization of a novel structure switching aptamer that was selected for total aflatoxin ( $\mathrm{AFB}_{1}, \mathrm{AFB}_{2}, \mathrm{AFG}_{1}, \mathrm{AFG}_{2}$ mixture). The aptamers were previously selected using an alternative method known as CaptureSELEX. The method was first developed to produce structure-switching signalling aptamers that require little optimization when incorporated into a fluorescent sensing platform. ${ }^{57}$ Computational predictions provided evidence that the DZA aptamer candidates have a structural bias that favours the formation of G-quadruplexes within their central domain. Moreover, EMSAs, typically used to study DNA-protein interactions, were utilized to support the selection of structure-switching aptamers. The optimal candidates were incorporated into a FRET-based hybridized sensing platform. Through the investigation of multiple FRET pairs and their incorporation into both aptamer and antisense capture probe.

Using FRET as a signal transduction method, a hybridized signalling platform was devised. As reported by Nutiu and Li (2005), the selection of structure-switching signalling aptamers do not require extensive optimization. A similar concept was adopted 
in the current study. Results were able to provide future insight into sensor designs and limitations of the current aptamers. The results suggest the high degree of secondary structure can greatly affect the chromophores that were incorporated into the sequences. The aptamers in question are comparatively long. Functional truncation of the sequences could be a viable option before considering alternate

Minimal sequence determination has been shown to provide several advantages. The elimination of superfluous nucleotides has been reported to increase the performance and binding affinity of aptamers. ${ }^{161}$ Shortening sequences by default, decreases the overall production cost of the aptamers as well. While is it possible for priming regions to be involved in ligand-binding, reports have suggested that constant primer-binding regions often do not contribute significantly to aptamer-target interactions. ${ }^{32,158}$ One of the first examples of this was the selection of an aptamer that binds and inhibits thrombin. The aptamer was selected from a pool of DNA containing a 60-nt random region which was flanked by two 18-nt primer regions. It was reported that a 15 -nt consensus sequence derived from the original 96-nt sequences retained the same activity. ${ }^{109}$ Removing unneeded nucleotides from the sequence could result in more consistent structure switching patterns if the aptamer is to be used. Modeling software such as UNAFold, and RNA structure that predict the secondary structure of aptamers are not completely accurate. Structure predictions are performed independently of ligand-induced structural changes. Secondary structure predictions can be used for guidance however, experimental 
approaches for sequences have proven to be effective. Frost $e t$ al. describe an approach for the rational design and testing of minimer sequences using DNAse I digestion.

Mckeague et al. reported an analytical comparison process that highlighted the inconsistencies in the many conventional aptamer affinity assays that are frequently used for characterization. ${ }^{65}$ It has been suggested that strategies that allow the binding characterization of many aptamers in a parallel manner would help the advancement of low throughput characterization. ${ }^{48}$ While MST is a suitable method for determining binding parameters of an aptamer that is free in solution. Many of the structural characterisation techniques that were used have been developed for determining binding affinities for aptamers in the past. ${ }^{162,163}$ In the future, these methods could be revisited for in order to obtain more comprehensive binding characteristics of the $\mathrm{AFB}_{1}$ aptamers. 


\section{Appendices}

\section{Appendix A : Melting Temperature Spectral Data}

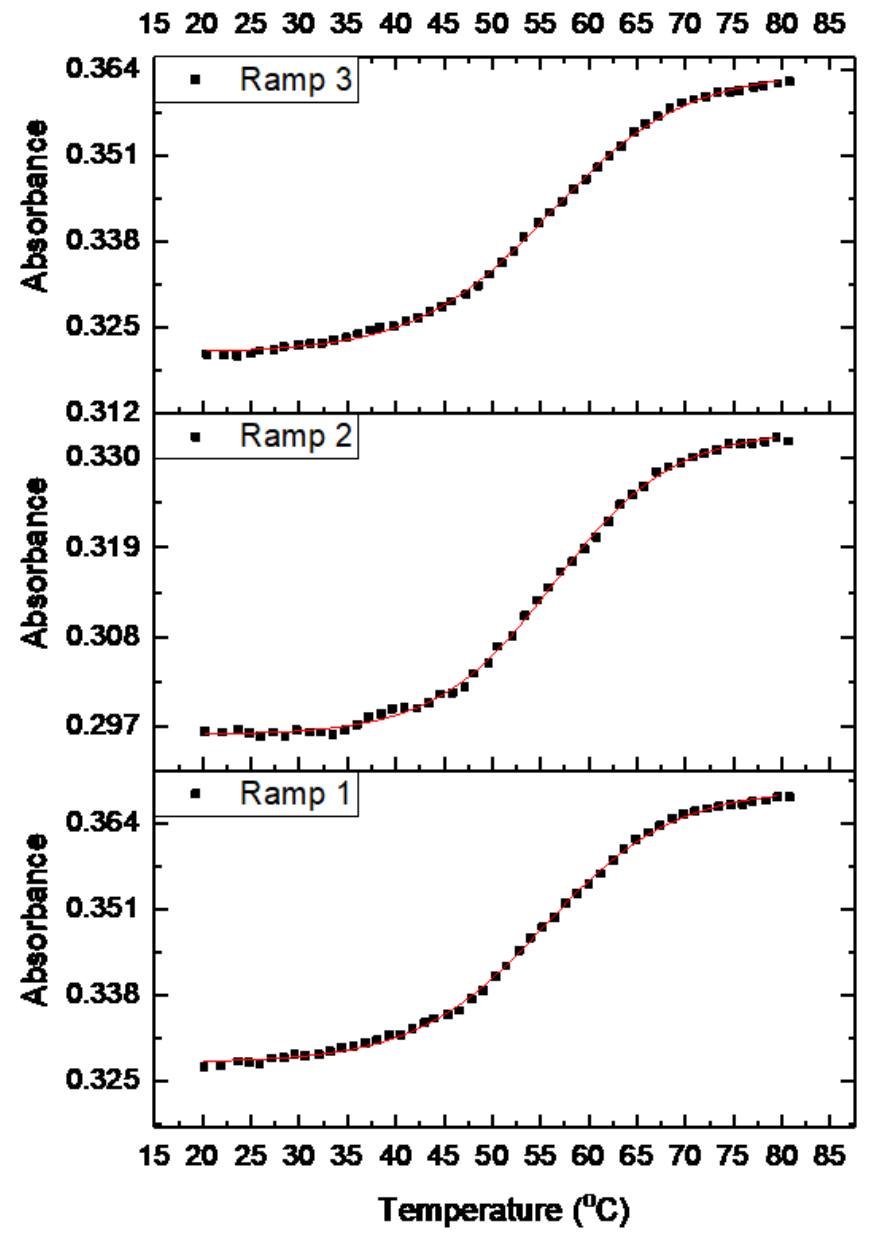

A.1 Melting temperature curve of hybridized DZA 3-FAM sequence and truncated capture probe, DZA CPnT-BHQ1 
Appendix B Electrophoretic mobility shift assay

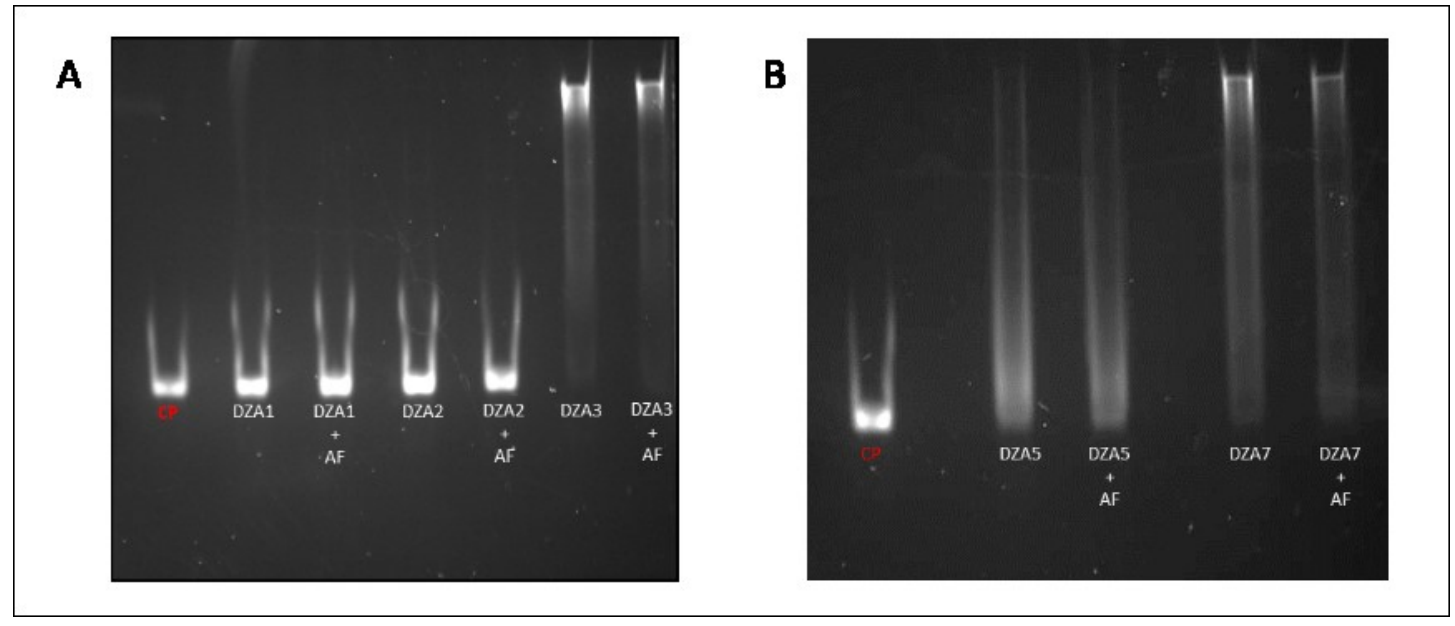

B.1 Fluorescent images of structure switching DZA aptamer candidates by electrophoretic mobility shift assay when challenged with total aflatoxin mixture.

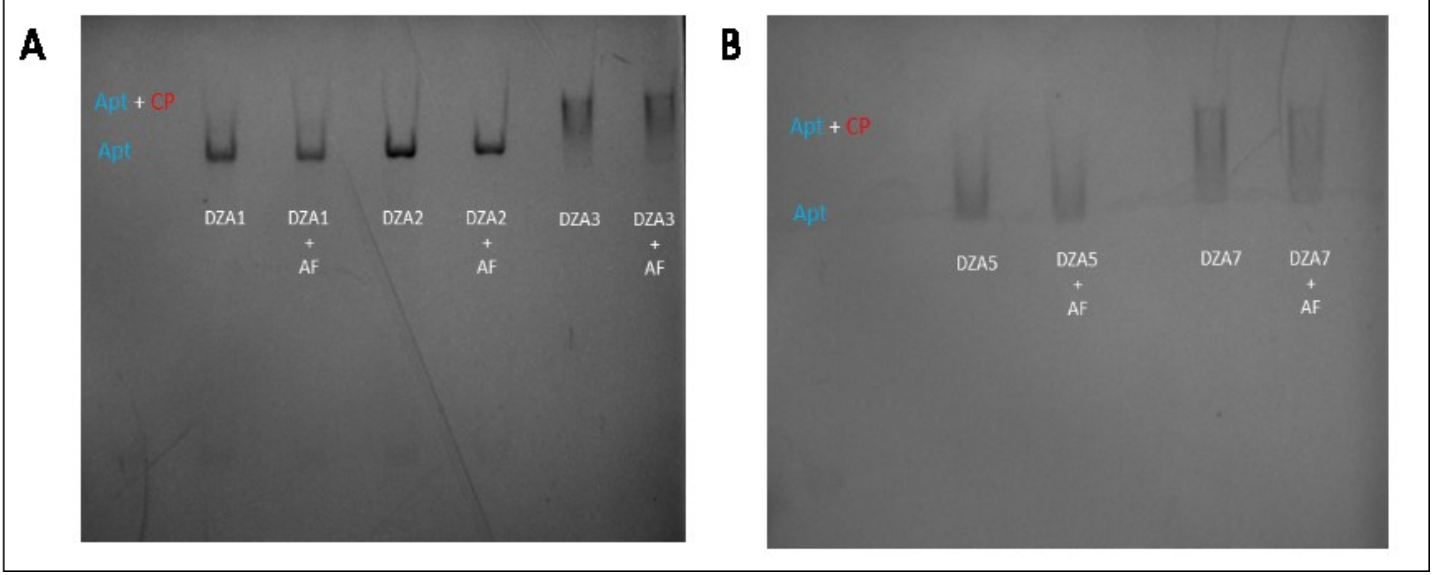

B.2 Epi-UV images of structure switching DZA aptamer candidates by electrophoretic mobility shift assay when challenged with total aflatoxin mixture. 


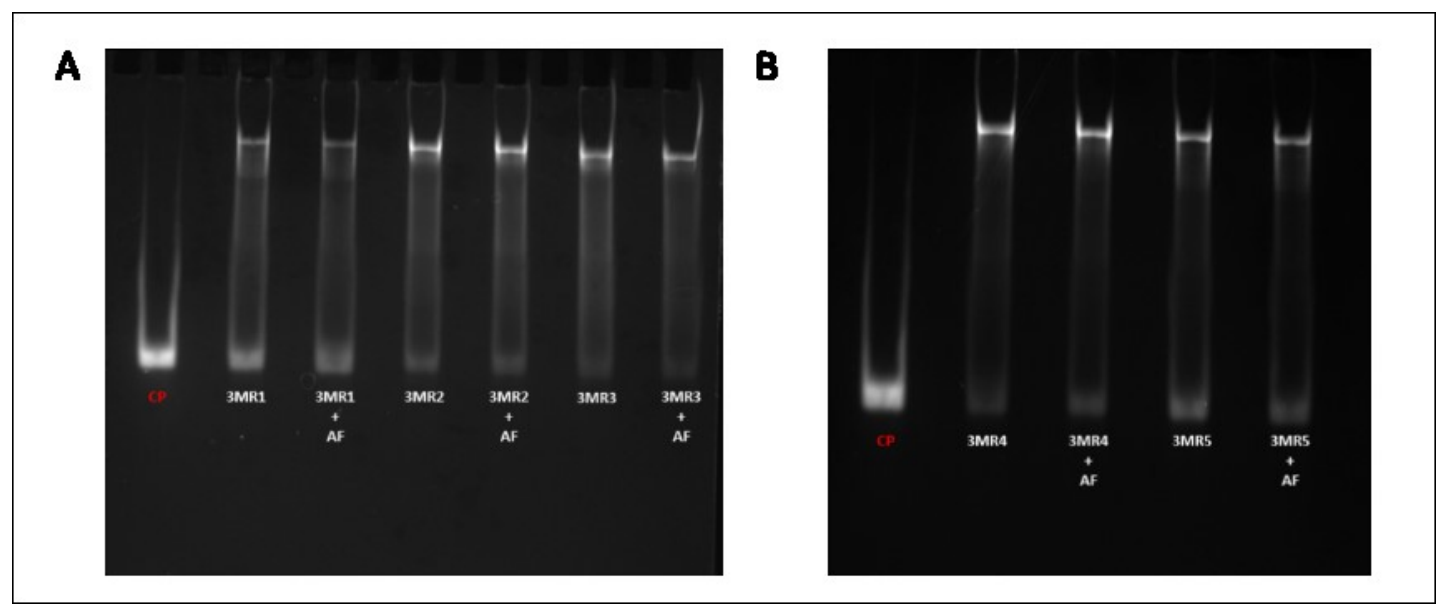

B.3 Fluorescent images of structure switching 3MR (random) aptamer candidates by electrophoretic mobility shift assay when challenged with total aflatoxin mixture.

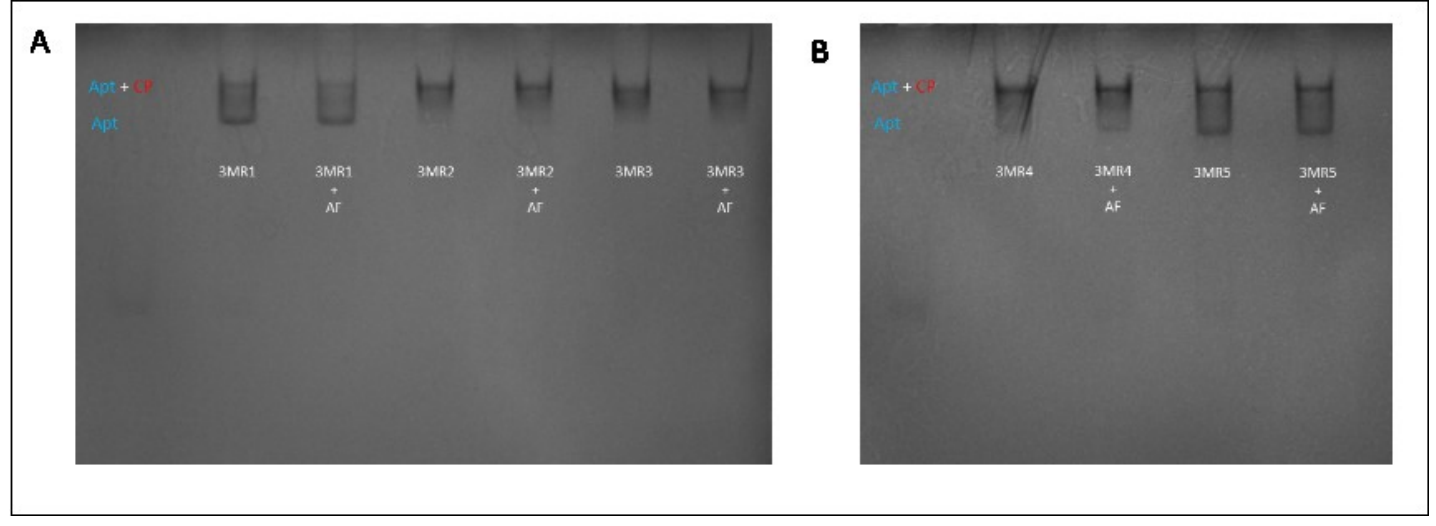

B.4 Epi-UV images of structure switching 3MR (random) aptamer candidates by electrophoretic mobility shift assay when challenged with total aflatoxin mixture. 
Appendix C Microscale thermophoresis data

A

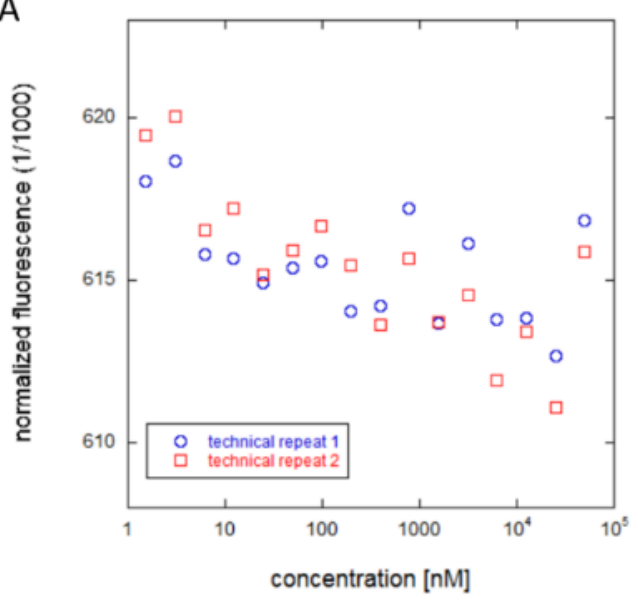

B

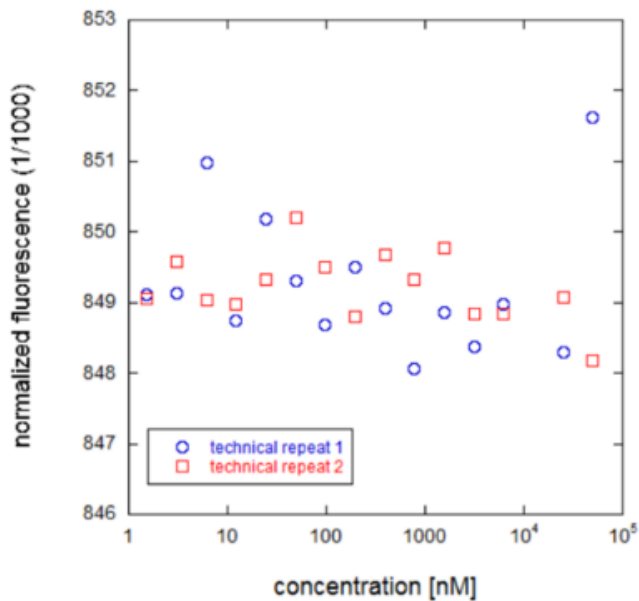

C.1 Microscale thermophoresis results for DZA3 challenged with $A) A_{2} B_{2}$ with no measurable binding constant between $1.5 \mathrm{nM}$ and $50 \mu \mathrm{M}$ B) $\mathrm{AFG}_{1}$ with no measurable binding constant between $1.5 \mathrm{nM}$ and $50 \mu \mathrm{M}$. 
A

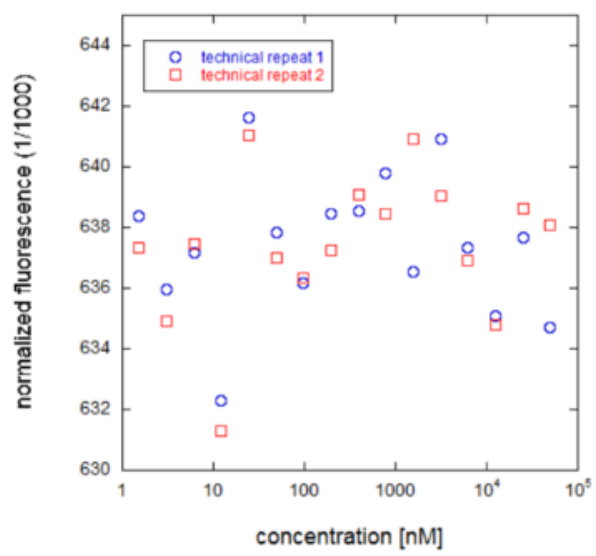

C

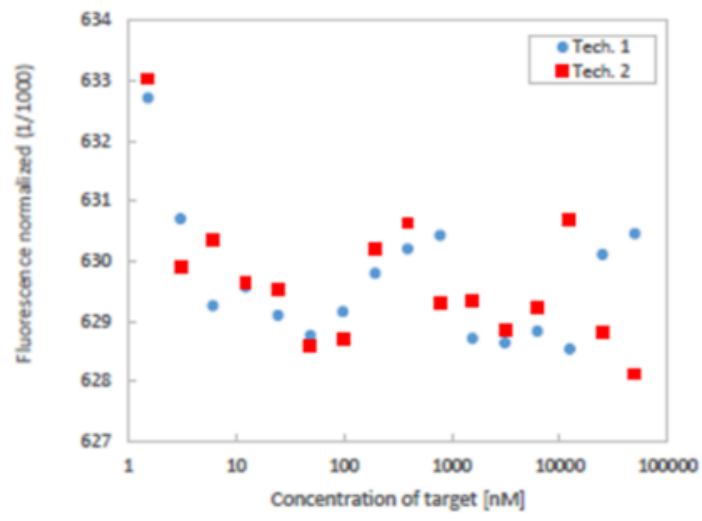

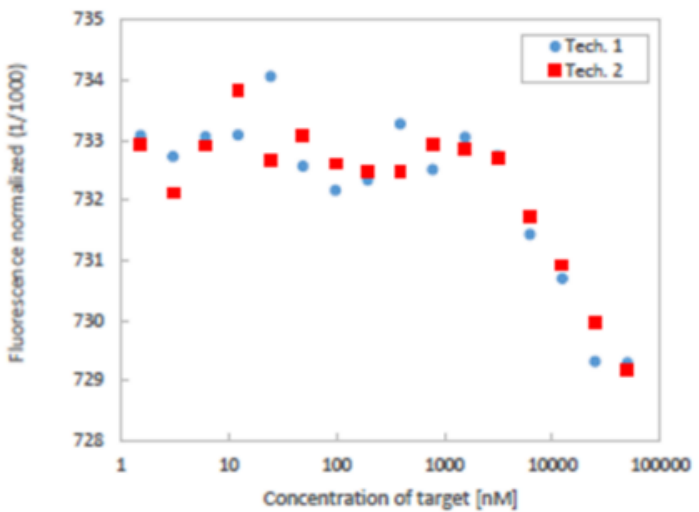

D

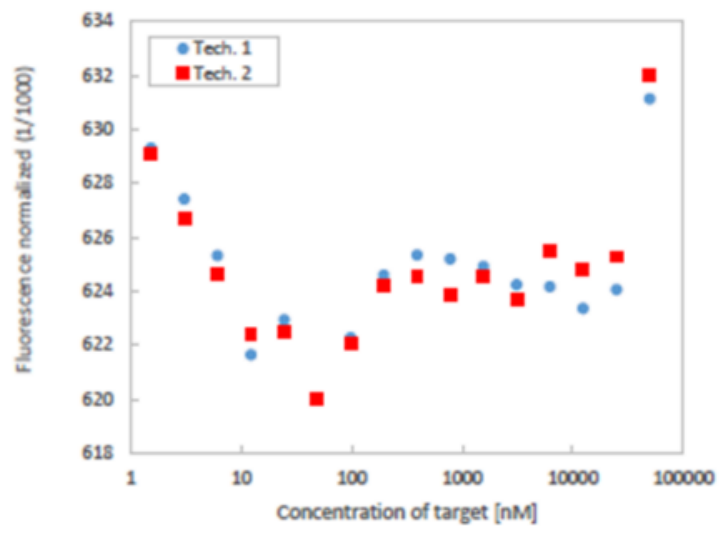

C.2 Microscale thermophoresis results for A) DZA5 challenged with $\mathrm{AFB}_{1}$ showing no measurable binding constant between $1.5 \mathrm{nM}$ and $50 \mu \mathrm{M}$ B) DZA5 challenged with $\mathrm{AFB}_{2}$ with no measurable binding constant between $1.5 \mathrm{nM}$ and $50 \mu \mathrm{M}$. C) DZA5 challenged with AFG1 with no measurable binding constant between $1.5 \mathrm{nM}$ and $50 \mu \mathrm{M}$. D) DZA5 challenged with $\mathrm{AFG}_{2}$ with no measurable binding constant between $1.5 \mathrm{nM}$ and $50 \mu \mathrm{M}$. 


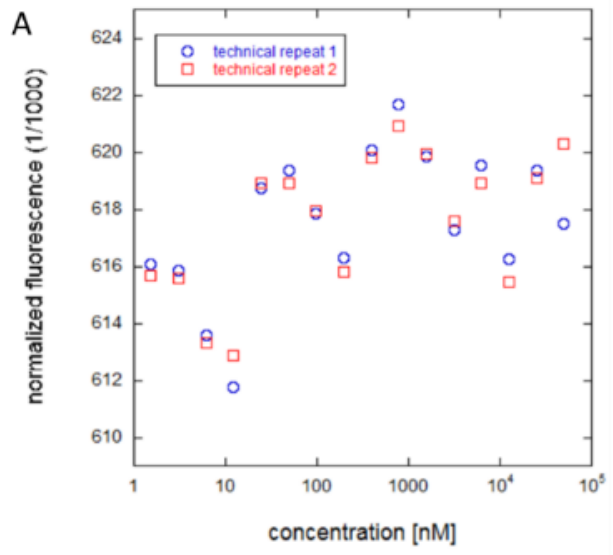

B

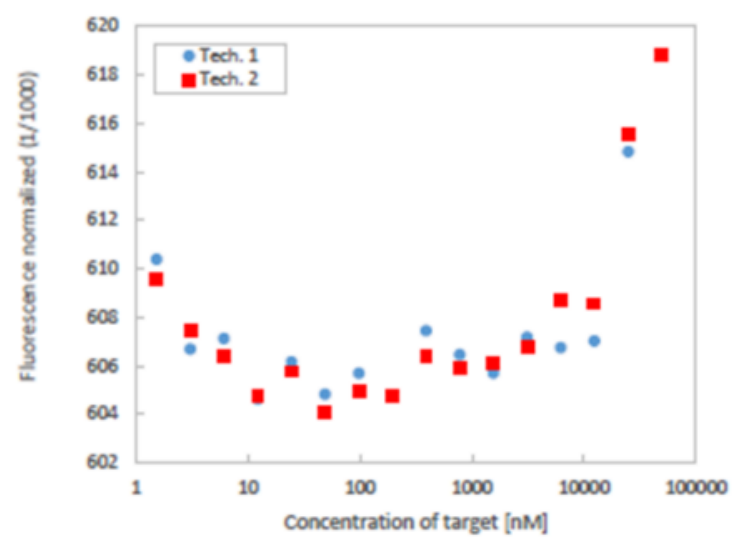

C

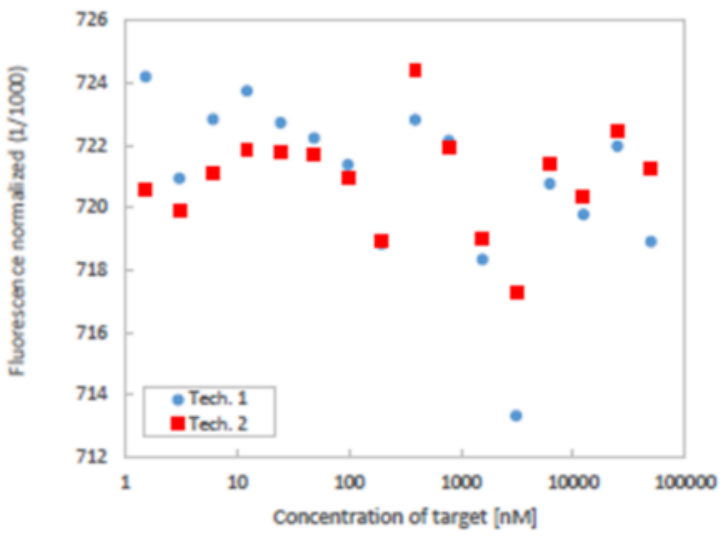

C.3 Microscale thermophoresis results for A) DZA7 challenged with $\mathrm{AFB}_{1}$ showing no measurable binding constant between $1.5 \mathrm{nM}$ and $50 \mu \mathrm{M}$ B) DZA5 challenged with $\mathrm{AFG}_{1}$ with no measurable binding constant between $1.5 \mathrm{nM}$ and $50 \mu \mathrm{M}$. C) DZA5 challenged with $\mathrm{AFG}_{2}$ with no measurable binding constant between $1.5 \mathrm{nM}$ and $50 \mu \mathrm{M}$. 


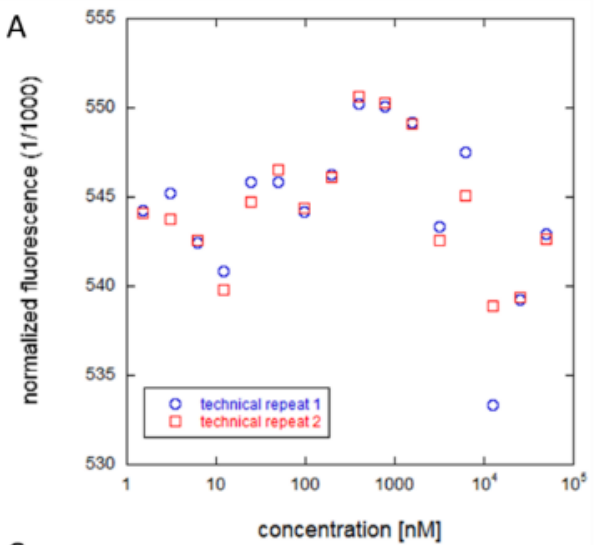

$c$

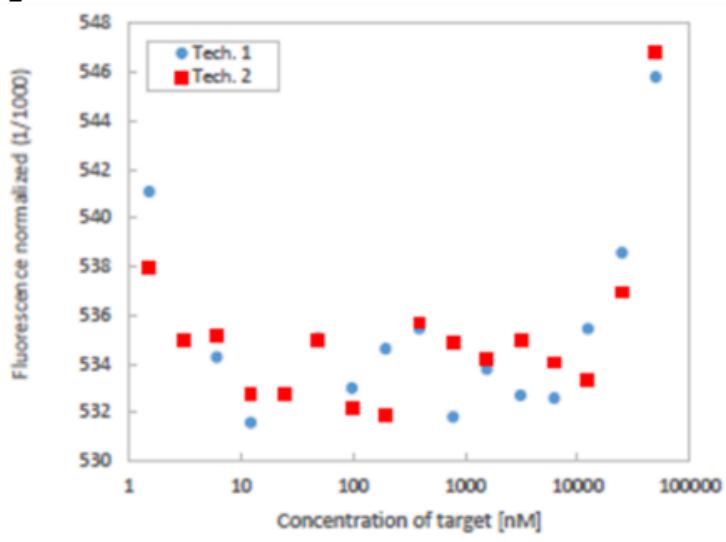

日

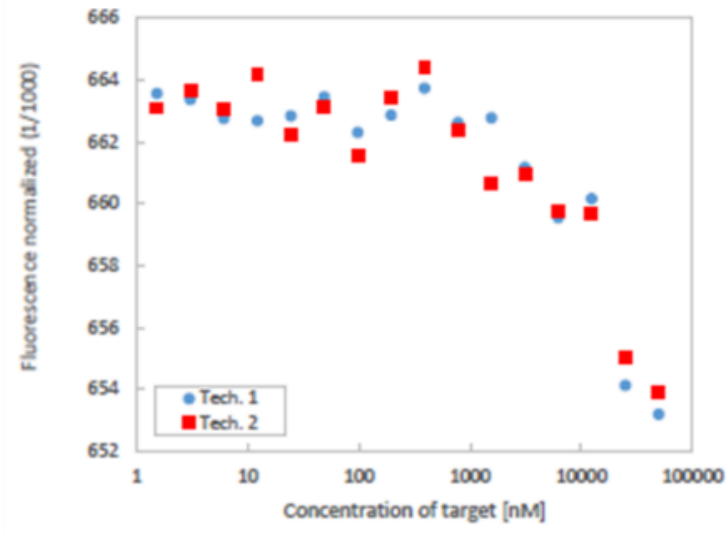

D

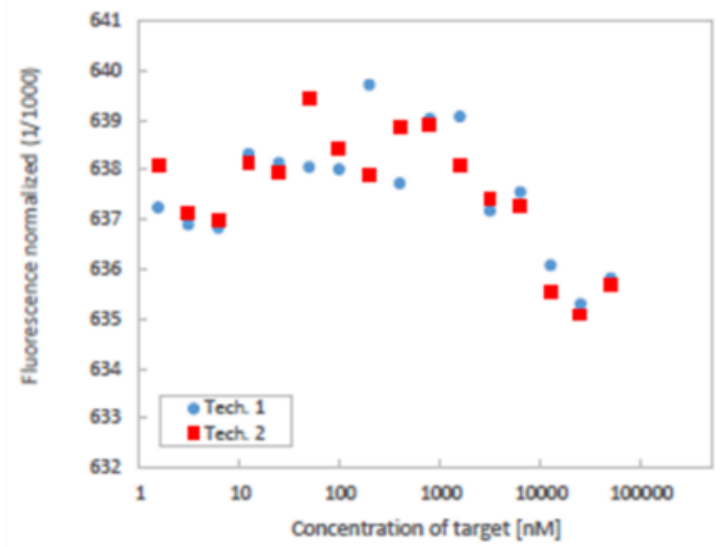

C.4 Microscale thermophoresis results for A) 3MR2 challenged with $\mathrm{AFB}_{1}$ showing no measurable binding constant between $1.5 \mathrm{nM}$ and $50 \mu \mathrm{M}$ B) $3 \mathrm{MR2}$ challenged with $\mathrm{AFB}_{2}$ with no measurable binding constant between $1.5 \mathrm{nM}$ and $50 \mu \mathrm{M} \mathrm{C}$ ) $3 \mathrm{MR2}$ challenged with $\mathrm{AFG}_{1}$ with no measurable binding constant between $1.5 \mathrm{nM}$ and $50 \mu \mathrm{M}$. D) $3 \mathrm{MR} 2$ challenged with $\mathrm{AFG}_{2}$ with no measurable binding constant between $1.5 \mathrm{nM}$ and $50 \mu \mathrm{M}$. 

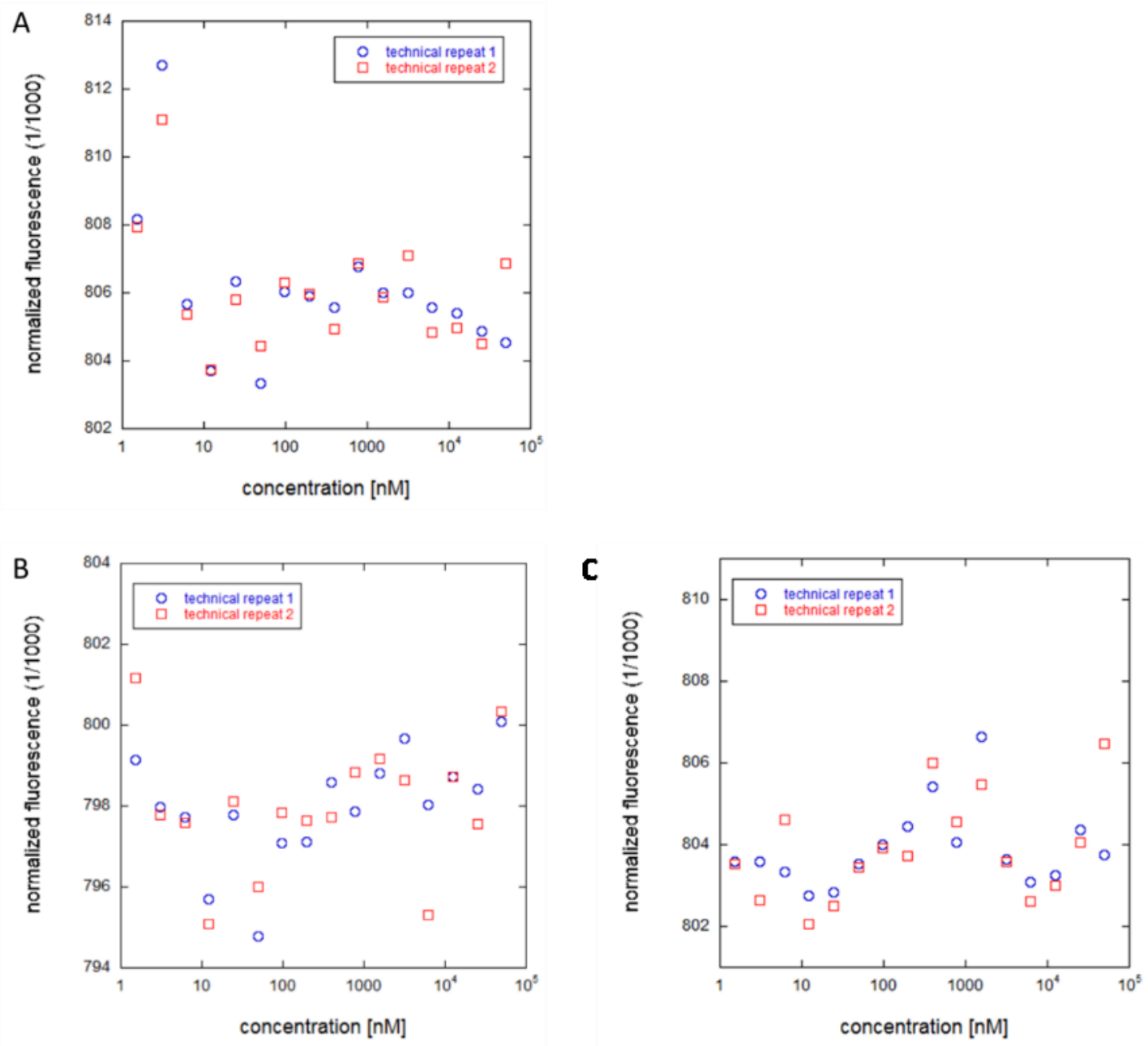

C.5 Microscale thermophoresis results for A) $3 \mathrm{MR5}$ challenged with $\mathrm{AFB}_{2}$ showing no measurable binding constant between $1.526 \mathrm{nM}$ and $50 \mu \mathrm{M}$ B) $3 \mathrm{MR5}$ challenged with AFG1 with no measurable binding constant between $1.526 \mathrm{nM}$ and $50 \mu \mathrm{M}$ C) $3 \mathrm{MR5}$ challenged with AFG2 with no measurable binding constant between $1.526 \mathrm{nM}$ and $50 \mu \mathrm{M}$. 


\section{Appendix D CD Spectral Data}

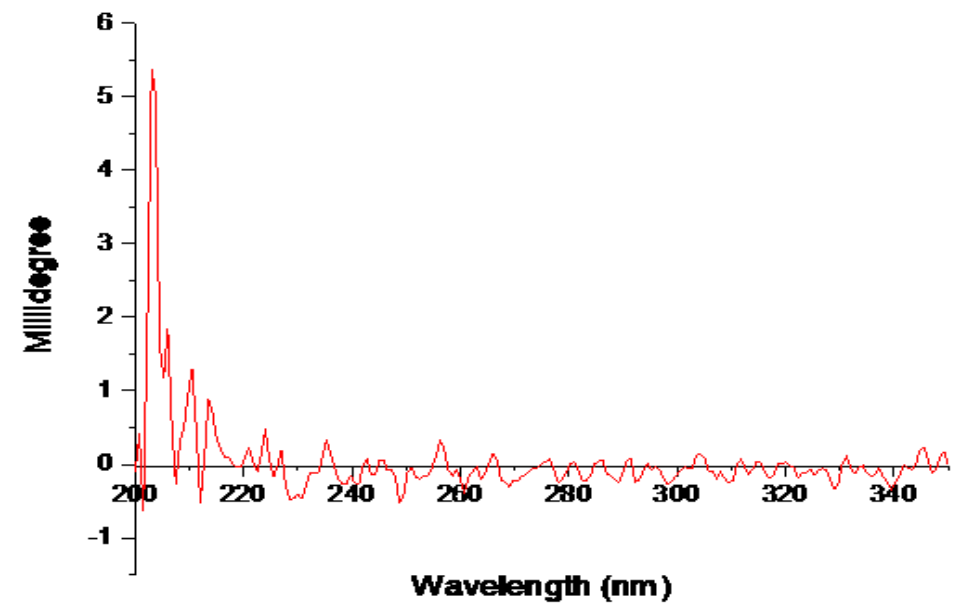

D.1 CD spectra of 3M Mg buffer demonstrating highly positive bands below $220 \mathrm{~nm}$.

Appendix E Spectral data for quenched aptamer assemblies challenged with AF 

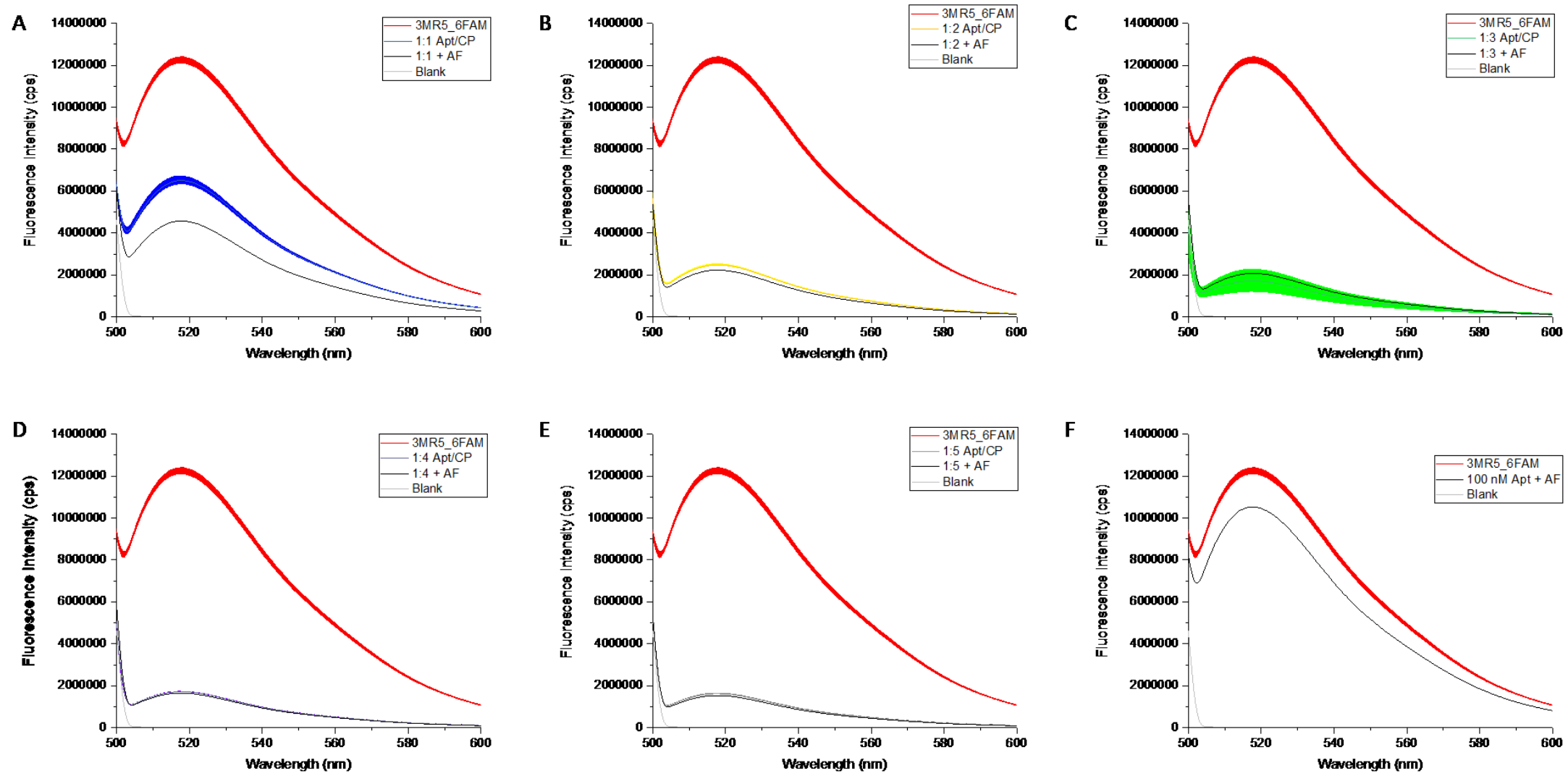

E.1 Fluorescence signal decrease with the addition of $1 \mu \mathrm{M} A F B_{1}$ to the quenched 3MR5_FAM signalling aptamer at varying Apt/CP ratios A) 1:1, B) 1:2, C) 1:3, D) 1:4, E) 1:5, F) 3MR5_FAM and AFB 1 only. The addition of aflatoxin results in further decreases in signal until maximal quenching is attained at higher ratios. 
A

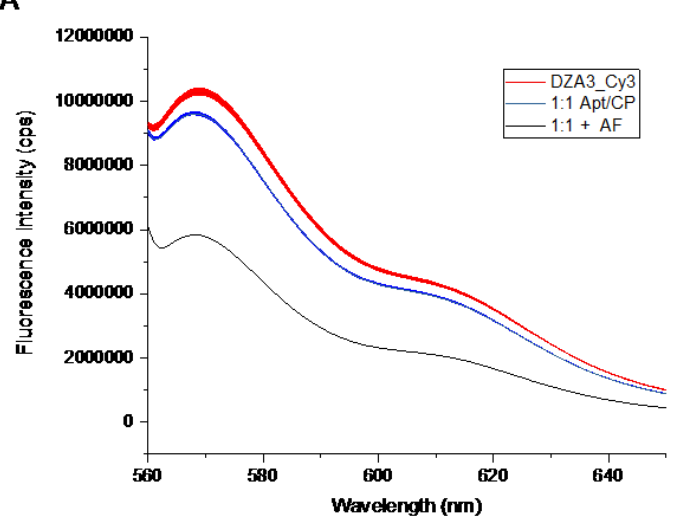

D

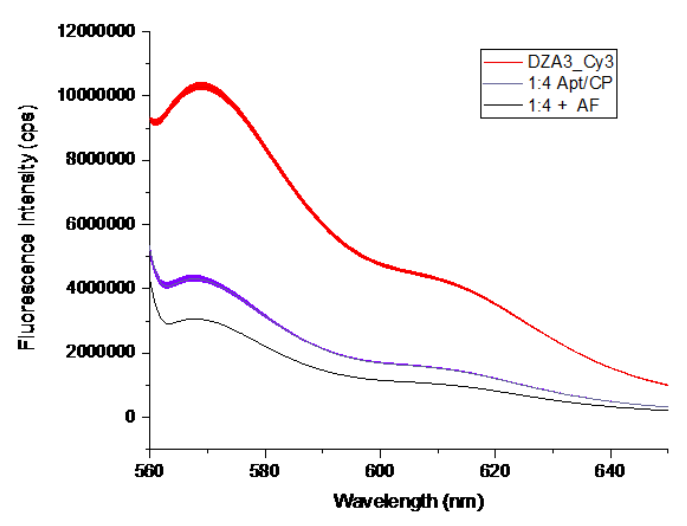

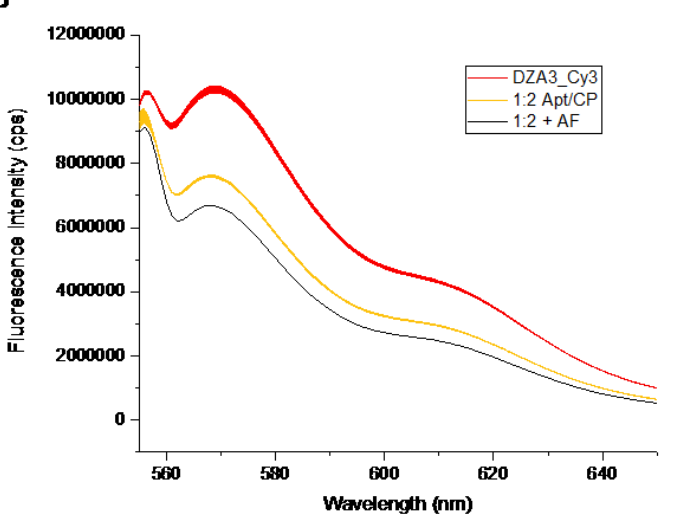

E

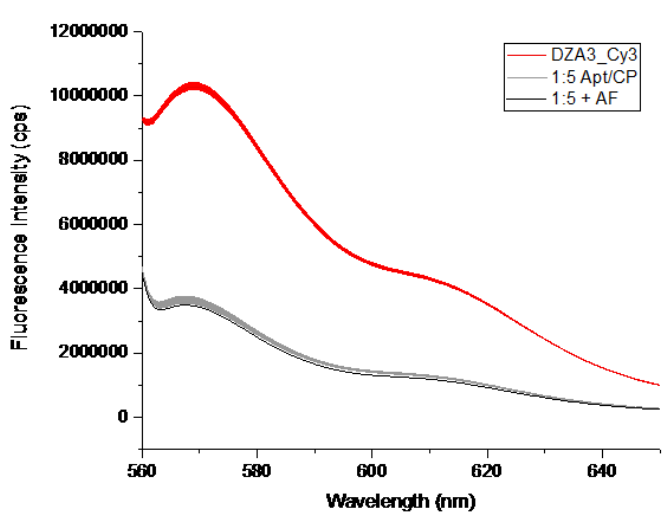

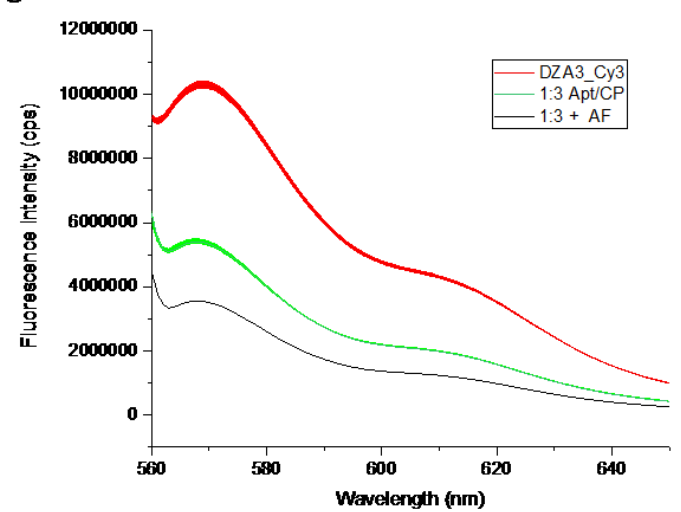

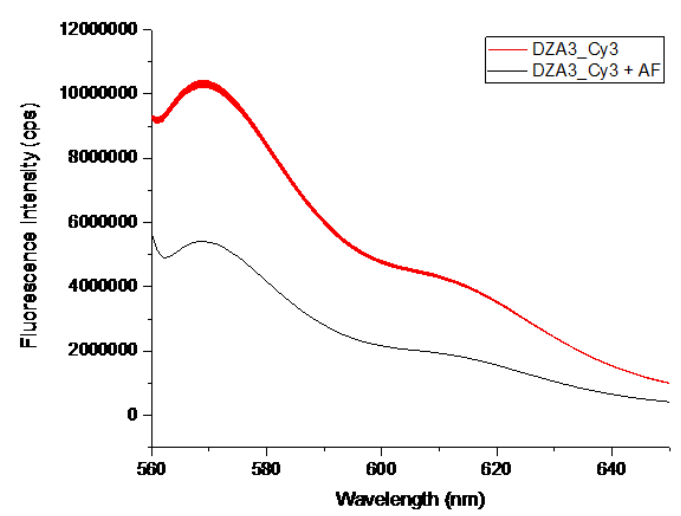

E.2 Fluorescence signal decrease with the addition of 1 $\mu \mathrm{M}$ AFB1 to the quenched DZA3_Cy3 signalling aptamer at varying Apt/CP ratios A) 1:1, B) 1:2, C) 1:3, D) 1:4, E) 1:5, F) DZA3_Cy3 and AFB1 only. The addition of aflatoxin results in further decreases in signal until maximal quenching is attained at higher ratios. 

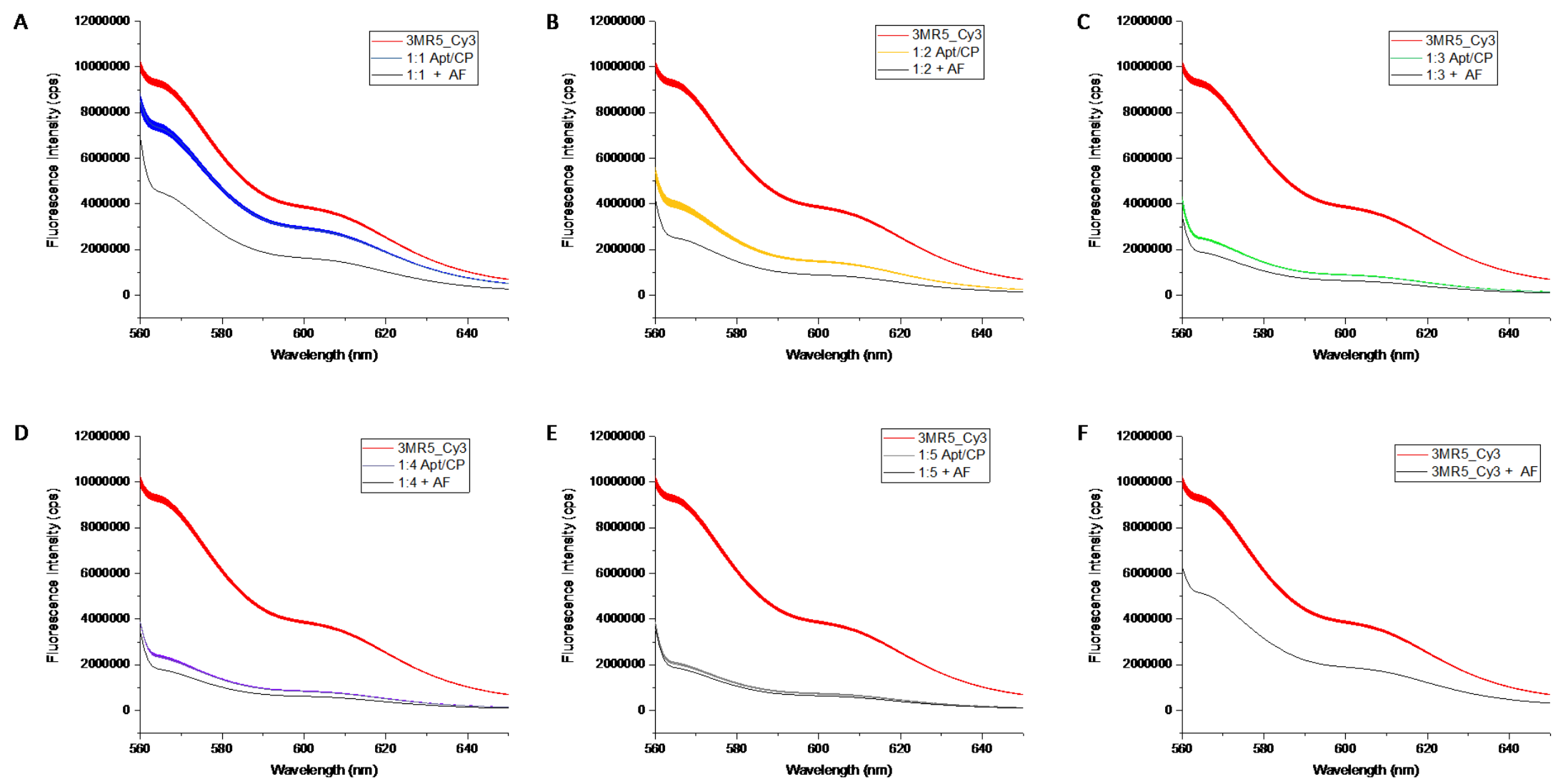

E.3 Fluorescence signal decrease with the addition of $1 \mu \mathrm{M}$ AFB1 to the quenched 3MR5_Cy3 signalling aptamer at varying Apt/CP ratios A) 1:1, B) 1:2, C) 1:3, D) 1:4, E) 1:5, F) 3MR5 Cy3 and AFB1 only. The addition of aflatoxin results in further decreases in signal until maximal quenching is attained at higher ratios. 


\section{References}

(1) Ji, C.; Fan, Y.; Zhao, L. Anim. Nutr. 2016, 2 (3), 127-133.

(2) Hussein, H. S.; Brasel, J. M. Toxicology 2001, 167 (2), 101-134.

(3) Moss, M. Mycol. Res. 1996, 100 (5), 513-523.

(4) Fox, E. M.; Howlett, B. J. Curr. Opin. Microbiol. 2008, 11 (6), 481-487.

(5) IARC. In Improving public health through pharmacy; Pitt, J. I., Wild, C. P., Baan, R. A., Wentzel, C. A., Gelderblom, W. C. ., Miller, J. D., Riley, R. T., Wu, F., Eds.; International Agency for Research on Cancer: Lyon, France, 2012; Vol. 158, pp 1-30.

(6) Marin, S.; Ramos, A. J.; Cano-Sancho, G.; Sanchis, V. Food Chem. Toxicol. 2013, 60, 218-237.

(7) Bennett, J. W.; Klich, M. Clin. Microbiol. Rev. 2003, 16 (3), 497-516.

(8) Peraica, M.; Radic Â, B.; Lucic, A.; Pavlovic, \& M. Bull. World Health Organ. 1999, 77 (9), 754-766.

(9) Pitt, J. I.; Miller, J. D. J. Agric. Food Chem. 2016, A-M.

(10) Marasas, W. F.; Kellerman, T. S.; Gelderblom, W. C.; Coetzer, J. A.; Thiel, P. G.; van der Lugt, J. J. Onderstepoort J. Vet. Res. 1988, 55 (4), 197-203.

(11) Cole, R. J. Mycotoxin Res. 1986, 2 (1), 3-7.

(12) Miller, J. D. J. stored Prod. Res 1995, 31 (1), 1-16.

(13) Cotty, P. J.; Jaime-Garcia, R. Int. J. Food Biol. 2007, 119, 109-115.

(14) Payne, G. A.; Brown, M. P. Annu. Rev. Phytopathol. 1998, 36, 329-362.

(15) Abrar, M.; Anjum, F. M.; Butt, M. S.; Pasha, I.; Randhawa, M. A.; Saeed, F.; Waqas, K. Crit. Rev. Food Sci. Nutr. 2013, 53 (8), 862-874.

(16) Villers, P. Front. Microbiol. 2014, 5 (158), 1-6.

(17) Miller, J. D. Food Addit. Contam. Part A 2008, 25 (2), 219-230.

(18) Crews, H.; Alink, G.; Andersen, R.; Braesco, V.; Holst, B.; Maiani, G.; Ovesen, L.; Scotter, M.; Solfrizzo, M.; Van Den Berg, R.; Verhagen, H.; Williamson, G. Br. J. Nutr. 2001, 86, 5-35.

(19) Wacoo, A. P.; Wendiro, D.; Vuzi, P. C.; Hawumba, J. F. J. Appl. Chem. 2014, 1-15.

(20) Lin, Y.-C.; Li, L.; Makarova, A. V; Burgers, P. M.; Stone, M. P.; Lloyd, R. S. Carcinogenesis 2014, 35 (7), 1461-1468.

(21) IARC. Monographs on the Evaluation of the Carcinogenic Risks to Humans. Overall Evaluations of Carcinogenicity: An Updating of IARC Monographs Volume 1 to 42; 1987.

(22) WHO and U. S. Centers for Disease Control and Prevention. Public Health Strategies for 
Preventing Aflatoxin Exposure; 2005.

(23) IARC. In IARC scientific publications; Pitt, J. I., Wild, C. P., Baan, R. A., Wentzel, C. A., Gelderblom, W. C. ., Miller, J. D., Riley, R. T., Wu, F., Eds.; 2012; pp 119-129.

(24) Hedayati, M. T.; Pasqualotto, A. C.; Warn, P. A.; Bowyer, P.; Denning, D. W. Microbiology 2007, 153, 1677-1692.

(25) Anklam, E.; Stroka, J.; Boenke, A. Food Control 2002, 173-183.

(26) Berthiller, F.; Brera, C.; Crews, C.; Iha, M. .; Lattanzio, V. M. T.; MacDonald, S.; Malone, R. J.; Maragos, C. M.; Solfrizzo, M.; Stroka, J.; Whitaker, T. B. World Mycotoxin J. 2015, $8(1), 5-36$.

(27) Stroka, J.; Maragos, C. M. World Mycotoxin J. 2016, 9 (5), 847-861.

(28) Krska, R.; Schubert-Ullrich, P.; Molinelli, A.; Sulyok, S.; MacDonald, S.; Crews, C. Food Addit. Contam. Part A 2008, 25 (2), 152-163.

(29) Weigand, J. E.; Suess, B. Appl. Microbiol. Biotechnol. 2009, 85, 229-236.

(30) Ellington, A. D.; Szostak, J. W. Nature 1990, 346, 818-822.

(31) Tuerk, C.; Gold, L. Science (80-. ). 1990, 249, 505-510.

(32) Huang, Z.; Szostak, J. W. RNA 2003, 9 (12), 1456-1463.

(33) Jayasena, S. D. Clin. Chem. 1999, 45, 1628-1650.

(34) Ruscito, A.; DeRosa, M. C. Front. Chem. 2016, 4 (14), 1-14.

(35) McKeague, M.; McConnell, E. M.; Cruz-Toledo, J.; Bernard, E. D.; Pach, A.; Mastronardi, E.; Zhang, X.; Beking, M.; Francis, T.; Giamberardino, A.; Cabecinha, A.; Ruscito, A.; Aranda-Rodriguez, R.; Dumontier, M.; DeRosa, M. C. J. Mol. Evol. 2015, 81 (5-6), 150161.

(36) Mirkin, S. M. Front. Biosci. 2008, 13, 1064-1071.

(37) Kypr, J.; Kejnovská, I.; Renč Iuk, D.; Vorlíč, M. Nucleic Acids Res. 2009, 37 (6), 17131725.

(38) Carothers, J. M.; Goler, J. A.; Kapoor, Y.; Lara, L.; Keasling, J. D. Nucleic Acids Res. 2010, 38 (8), 2736-2747.

(39) Keefe, A. D.; Pai, S.; Ellington, A. Nat. Rev. Drug Discov. 2010, 9, 537-550.

(40) Stoltenburg, R.; Reinemann, A. C.; Strehlitz, A. B. Anal. Bioanal. Chem. 2005, 383, 8391.

(41) Vorličková, M.; Kejnovská, I.; Bednářová, K.; Renčiuk, D.; Kypr, J. Chirality 2012, 24 (9), 691-698.

(42) Ehrentreich-Förster, E.; Orgel, D.; Krause-Griep, A.; Cech, B.; Erdmann, V. a; Bier, F.; Scheller, F. W.; Rimmele, M. Anal. Bioanal. Chem. 2008, 391, 1793-1800.

(43) Chakravarthy, U.; Adamis, A. P.; Cunningham, E. T. J.; Goldbaum, M.; Guyer, D. R.; Katz, B.; Patel, M. Ophthalmology 2006, 113 (9), 1-25.

(44) Gragoudas, E. S.; Adamis, A. P.; Cunningham, E. T.; Feinsod, M.; Guyer, D. R. N. Engl. J. 
Med. 2004, 351 (27), 2805-2816.

(45) Mcbride, L. J.; Caruthers, N. H. Tetrahedron Lett. 1983, 24 (3), 245-248.

(46) Mullis, K. B. Angew. Chemie Int. Ed. English 1994, 33 (12), 1209-1213.

(47) Walter, J. G.; Stahl, F.; Scheper, T. Eng. Life Sci. 2012, 12 (5), 496-506.

(48) Cho, M.; Oh, S. S.; Nie, J.; Stewart, R.; Eisenstein, M.; Chambers, J.; Marth, J. D.; Walker, F.; Thomson, J. A.; Soh, H. T. PNAS 2013, 110 (46), 18460-18465.

(49) Rhouati, A.; Yang, C.; Hayat, A.; Marty, J. L. Toxins (Basel). 2013, 5 (11), 1988-2008.

(50) Kubik, M. F.; Stephens, A. W.; Schneider, D.; Marlar, R. A.; Tasset, D. Nucleic Acids Res. 1994, 22 (13), 2619-2626.

(51) Mendonsa, S. D.; Bowser, M. T. J. Am. Chem. Soc. 2005, 127 (26), 9382-9383.

(52) Blank, M.; Weinschenk, T.; Priemer, M.; Schluesener, H. J. Biol. Chem. 2001, 276 (19), 16464-16468.

(53) Zhang, F.; Anderson, D. J. Biol. Chem. 1998, 273 (5), 2947-2953.

(54) Chandra, S.; Gopinath, B. Anal Bioanal Chem 2007, 387, 171-182.

(55) McKeague, M.; DeRosa, M. C. J. Nucleic Acids 2012, 1-20.

(56) Wilson, C.; Szostak, J. W. Chem. Biol. 1998, 5 (11), 5609-5617.

(57) Nutiu, R.; Li, Y. Angew. Chemie 2005, 44 (7), 1061-1065.

(58) Stoltenburg, R.; Nikolaus, N.; Strehlitz, B. J. Anal. Methods Chem. 2012, 1-14.

(59) Oh, S. S.; Plakos, K.; Xiao, Y.; Eisenstein, M.; Soh, H. T. ACS Nano 2013, 7 (11), 96759683.

(60) Jing, M.; Bowser, M. T. Anal. Chim. Acta 2011, 686 (2), 9-18.

(61) Flinders, J.; DeFina, S. C.; Brackett, D. M.; Baugh, C.; Wilson, C.; Dieckmann, T. ChemBioChem 2004, 5 (1), 62-72.

(62) Cruz-Aguado, J.; Penner, G. J. Agric. Food Chem. 2008, 56, 10456-10461.

(63) Potty, A. S. R.; Kourentzi, K.; Fang, H.; Jackson, G. W.; Zhang, X.; Legge, G. B.; Willson, R. C. 2008.

(64) Oh, S. S.; Plakos, K.; Lou, X.; Xiao, Y.; Soh, H. T. Proc. Natl. Acad. Sci. U. S. A. 2010, 107 (32), 14053-14058.

(65) McKeague, M.; Velu, R.; De Girolamo, A.; Valenzano, S.; Pascale, M.; Smith, M.; Derosa, M. C. Toxins (Basel). 2016, 8 (336), 1-13.

(66) White, R. J.; Rowe, A. A.; Plaxco, K. W. Analyst 2010, 135 (3), 589-594.

(67) Ma, X.; Wang, W.; Chen, X.; Xia, Y.; Wu, S.; Duan, N.; Wang, Z. Eur. Food Res. Technol. 2014, 238, 919-925.

(68) Malhotra, S.; Pandey, A. K.; Rajput, Y. S.; Sharma, R. J. Mol. Recognit. 2014, 27 (8), 493500 .

(69) Ma, X.; Wang, W.; Chen, X.; Xia, Y.; Duan, N.; Wu, S.; Wang, Z. Food Control 2015, 47, 
$545-551$.

(70) Chryseis Le, L.; Cruz-Aguado, J.; Penner, G. A. DNA ligands for aflatoxins and zearalenone. PCT/CA2010/001292, 2011.

(71) Nguyen, B. H.; Tran, L. D.; Do, Q. P.; Nguyen, H. Le; Tran, N. H.; Nguyen, P. X. Mater. Sci. Eng. C 2013, 33, 2229-2234.

(72) Chambers, J. P.; Arulanandam, B. P.; Matta, L. L.; Weis, A.; Valdes, J. J. Curr. Issues Mol. Biol. 2008, 10 (2), 1-12.

(73) Cho, E. J.; Lee, J.-W.; Ellington, A. D. Annu. Rev. Anal. Chem. 2009, 2, 241-264.

(74) Jhaveri, S.; Rajendran, M.; Ellington, A. D. Nat. Biotechnol. 2000, 18, 1293-1297.

(75) Guo, X.; Wen, F.; Zheng, N.; Luo, Q.; Wang, H. Biosens. Bioelectron. 2014, 56, 340-344.

(76) Sun, L.; Wu, L.; Zhao, Q. Microchim. Acta 2017, 1-6.

(77) Zheng, W.; Teng, J.; Cheng, L.; Ye, Y.; Pan, D.; Wu, J.; Xue, F.; Liu, G.; Chen, W. Biosens. Bioelectron. 2016, 80, 574-581.

(78) Abnous, K.; Danesh, N. M.; Alibolandi, M.; Ramezani, M.; Sarreshtehdar Emrani, A.; Zolfaghari, R.; Taghdisi, S. M. Biosens. Bioelectron. 2017, 94, 374-379.

(79) Ma, Y.; Mao, Y.; Huang, D.; He, Z.; Yan, J.; Tian, T.; Shi, Y.; Song, Y.; Li, X.; Zhu, Z.; Zhou, L.; Yang, C. J. Lab Chip 2016, 115, 73-83.

(80) Jhaveri, S. D.; Kirby, R.; Conrad, R.; Maglott, E. J.; Bowser, M.; Kennedy, R. T.; Glick, G.; Ellington, A. D. 1999.

(81) Goudreau, D. N.; Smith, M.; McConnell, E. M.; Ruscito, A.; Velu, R.; Callahan, J.; DeRosa, M. C. In Sensing Techniques for Food Safety and Quality Control; The Royal Society of Chemistry, 2017; pp 200-271.

(82) Chen, L.; Wen, F.; Li, M.; Guo, X.; Li, S.; Zheng, N.; Wang, J. Food Chem. 2017, 215 , 377-382.

(83) Joo, M.; Baek, S. H.; Cheon, S. A.; Chun, H. S.; Choi, S.-W.; Park, T. J. Colloids Surfaces B Biointerfaces 2017, 154, 27-32.

(84) Goud, K. Y.; Sharma, A.; Hayat, A.; Catanante, G.; Gobi, K. V.; Gurban, A. M.; Marty, J. L. Anal. Biochem. 2016, 508, 19-24.

(85) Guo, X.; Wen, F.; Zheng, N.; Li, S.; Fauconnier, M. L.; Wang, J. Anal. Bioanal. Chem. 2016, 408 (20), 5577-5584.

(86) Huang, H.; Qin, J.; Hu, K.; Liu, X.; Zhao, S.; Huang, Y. RSC Adv. 2016, 6 (89), 8604386050 .

(87) Wang, B.; Chen, Y.; Wu, Y.; Weng, B.; Liu, Y. Biosens. Bioelectron. 2016, 78, $23-30$.

(88) Yugender Goud, K.; Catanante, G.; Hayat, A.; M., S.; Vengatajalabathy Gobi, K.; Marty, J. L. Sensors Actuators, B Chem. 2016, 235, 466-473.

(89) Zhang, J.; Xia, Y.-K.; Chen, M.; Wu, D.-Z.; Cai, S.-X.; Liu, M.-M.; He, W.-H.; Chen, J.-H. 
Sensors Actuators B Chem. 2016, 235, 79-85.

(90) Castillo, G.; Spinella, K.; Poturnayová, A.; Šnejdárková, M.; Mosiello, L.; Hianik, T. Food Control 2015, 52 (165), 9-18.

(91) Jiang, M.; Braiek, M.; Florea, A.; Chrouda, A.; Farre, C.; Bonhomme, A.; Bessueille, F.; Vocanson, F.; Zhang, A.; Jaffrezic-Renault, N. Toxins (Basel). 2015, 7 (9), 3540-3553.

(92) Lu, Z.; Chen, X.; Wang, Y.; Zheng, X.; Li, C. M. Microchim. Acta 2015, 182 (4), 571-578.

(93) Seok, Y.; Byun, J. Y.; Shim, W. B.; Kim, M. G. Anal. Chim. Acta 2015, 886, 182-187.

(94) Zhao, Y.; Yang, Y.; Luo, Y.; Yang, X.; Li, M.; Song, Q. ACS Appl. Mater. Interfaces 2015, 7 (39), 21780-21786.

(95) Guo, X.; Wen, F.; Zheng, N.; Luo, Q.; Wang, H.; Wang, H.; Li, S.; Wang, J. Biosens. Bioelectron. 2014, 56, 340-344.

(96) Shim, W. B.; Kim, M. J.; Mun, H.; Kim, M. G. Biosens. Bioelectron. 2014, 62, $288-294$.

(97) Shim, W.-B.; Mun, H.; Joung, H.-A.; Ofori, J. A.; Chung, D.-H.; Kim, M.-G. Food Control 2014, 36 (1), 30-35.

(98) Liu, J.-L.; Zhao, M.; Zhuo, Y.; Chai, Y.-Q.; Yuan, R. Chem. - A Eur. J. 2017, 23 (8), 1853-1859.

(99) Istamboulié, G.; Paniel, N.; Zara, L.; Granados, L. R.; Barthelmebs, L.; Noguer, T. Talanta 2016, 146, 464-469.

(100) Liu, W.; Zhu, H.; Zheng, B.; Cheng, S.; Fu, Y.; Li, W.; Lau, T.-C.; Liang, H. Nucleic Acids Res. 2012, 40 (9), 4229-4236.

(101) Travascio, P.; Li, Y.; Sen, D. Chem. Biol. 1998, 5 (9), 505-517.

(102) Marchand, A.; Erie Gabelica, V. Nucleic Acids Res. 2016, 44 (10), 10999-11012.

(103) Van Mourik, T.; Dingley, A. J. Chem. - A Eur. J. 2005, 11 (20), 6064-6079.

(104) Burge, S.; Parkinson, G. N.; Hazel, P.; Todd, A. K.; Neidle, S. Nucleic Acids Res. 2006, 34 (19), 5402-5415.

(105) Dingley, A. J.; Peterson, R. D.; Grzesiek, S.; Feigon, J. J. Am. Chem. Soc. 2005, 127 (41), 14466-14472.

(106) Kikin, O.; D’Antonio, L.; Bagga, P. S. Nucleic Acids Res. 2006, 34, W676-W682.

(107) Azargun, M.; Fridgen, T. D. Phys. Chem. Chem. Phys. 2015, 17 (39), 25778-25785.

(108) Juskowiak, B. Anal Bioanal Chem 2010, 399, 3157-3176.

(109) Bock, L. C.; Griffin, L. C.; Latham, J. A.; Vermaas, E. H.; Toole, J. L. Nature 1992, 355, 564-566.

(110) Todd, A. K.; Johnston, M.; Neidle, S. Nucleic Acids Res. 2005, 33 (9), 2901-2907.

(111) Huppert, J. L.; Balasubramanian, S. Nucleic Acids Res. 2005, 33 (9), 2908-2916.

(112) Karsisiotis, A. I.; Hessari, N. M. A.; Novellino, E.; Spada, G. P.; Randazzo, A.; Webba Da 
Silva, M. Angew. Chemie - Int. Ed. 2011, 50 (45), 10645-10648.

(113) Gray, D. M.; Wen, J.-D.; Gray, C. W.; Repges, R.; Repges, C.; Raabe, G.; Fleischhauer, J. Chirality 2008, 20 (3-4), 431-440.

(114) Entzian, C.; Schubert, T. Methods 2016, 27-34.

(115) Braun, D.; Libchaber, A. Am. Phys. Soc. 2002, 89 (18), 1-4.

(116) Maxam, a M.; Gilbert, W. Proc. Natl. Acad. Sci. U. S. A. 1977, 74 (2), 560-564.

(117) Lau, P. S.; Coombes, B. K.; Li, Y. 2010, 65, 7938-7942.

(118) Munzar, J. D.; Ng, A.; Corrado, M.; Juncker, D. Chem. Sci. 2017, 8, 2251-2256.

(119) Fried, M.; Crothers, D. M. Nucleic Acids Res. 1981, 9, 6505-6525.

(120) Tinoco, I. J. Am. Chem. Soc. 1960, 82, 4785-4790.

(121) D’Abramo, M.; Lara Castellazzi, C.; Orozco, M.; Amadei, A. J. Phys. Chem. B 2013, 117, 8697-8704.

(122) Rachwal, P. A.; Fox, K. R. Methods 2007, 43 (4), 291-301.

(123) Mergny, J.-L.; Phan, A.-T.; Lacroix, L. FEBS Lett. 1998, 435 (1), 74-78.

(124) Risitano, A.; Fox, K. R. Nucleic Acids Res. 2004, 32 (8), 2598-2606.

(125) Smaldino, P. J.; Routh, E. D.; Kim, J. H.; Giri, B.; Creacy, S. D.; Hantgan, R. R.; Akman, S. A.; Vaughn, J. P.; Pj, S.; Ed, R.; Jh, K.; Giri, B.; Sd, C.; Rr, H. PLoS One 2015, 10 (7).

(126) Hazel, P.; Huppert, J.; Balasubramanian, S.; Neidle, S. J. Am. Chem. Soc. 2004, 126, $16405-16415$.

(127) Vorlíčková, M.; Bednářová, K.; Kejnovská, I.; Kypr, J. Biopolymers 2007, 86 (1), 1-10.

(128) Boehr, D. D.; Nussinov, R.; Wright, P. E. Nat Chem Biol 2009, 5 (11), 789-796.

(129) Cunningham, P. D.; Khachatrian, A.; Buckhout-White, S.; Deschamps, J. R.; Goldman, E. R.; Medintz, I. L.; Melinger, J. S. J. Phys. Chem. B 2014, 118, 14555-14565.

(130) Marras, S. a E. Methods Mol. Biol. 2006, 335, 3-16.

(131) Fluorescent Energy Transfer Nucleic Acid Probes, 1st ed.; Didenko, V. V., Ed.; Humana Press: Totowa, New Jersey, 2006.

(132) Forster, T. Discuss. Faraday Soc. 1959, 27, 7-17.

(133) Tyagi, S.; Kramer, F. R. Nat. Biotechnol. 1995, 14, 303-308.

(134) Le Reste, L.; Hohlbein, J.; Gryte, K.; Kapanidis, A. N. Biophys. J. 2012, 102, 2658-2668.

(135) Yin, Y.; Xin, A.; Zhao, S. Acc. Chem. Res. 1172, 44 (11), 1172-1181.

(136) Goodman, R. P.; Schaap, I. A. T.; Tardin, C. F.; Erben, C. M.; Berry, R. M.; Schmidt, C. F.; Turberfield, A. J. Science (80-. ). 2005, 310, 1661-1665.

(137) Gartner, Z. J.; Tse, B. N.; Grubina, R.; Doyon, J. B.; Snyder, T. M.; Liu, D. R. Science. 
2004, 305 (5690), 1-9.

(138) Zhang, Y.; Hong, H.; Cai, W. Curr. Med. Chem. 2011, 18 (27), 4185-4194.

(139) Sharma, A.; Catanante, G.; Hayat, A.; Istamboulié, G.; Rejeb, I. Ben; Bhand, S.; Marty, J.L. Talanta 2016, 158, 35-41.

(140) Wang, B.; Wu, Y.; Chen, Y.; Weng, B.; Xu, L.; Li, C. Biosens. Bioelectron. 2016, 81, $125-130$.

(141) Gu, C.; Lan, T.; Shi, H.; Lu, Y. Anal. Chem. 2015, 87, 7676-7682.

(142) Porchetta, A.; Vallée-Bélisle, A.; Plaxco, K. W.; Ricci, F. J. Am. Chem. Soc. 2012, 134 (51), 20601-20604.

(143) Johansson, M. K. Methods Mol. Biol. 2006, 335, 17-29.

(144) Nutiu, R.; Li, Y. J. Am. Chem. Soc. 2002, 125, 4771-4778.

(145) Rudert, W. Biotechniques 1997, 22, 1140-1145.

(146) Liu, C.; Chen, J.; Mao, G.; Su, C.; Ji, X.; He, Z. Anal. Methods 2016, 8 (30), 5957-5961.

(147) Johansson, M. K.; Fidder, H.; Dick, D.; Cook, R. M. J. Am. Chem. Soc. 2002, 124 (24), 6950-6956.

(148) Seidel, C. A. M.; And, A. S.; Sauer, M. H. M. J. Phys. Chem. 1996, 100 (13), 5541-5553.

(149) Heinlein, T.; Knemeyer, J.-P.; Piestert, O.; Sauer, M. 2003.

(150) Zhou, H.; Xie, S.-J.; Li, J.-S.; Wu, Z.-S.; Shen, G.-L. Chem. Commun. 2012, 48 (48), 10760-10762.

(151) Yang, X.; Zhu, Y.; Liu, P.; He, L.; Li, Q.; Wang, Q.; Wang, K.; Huang, J.; Liu, J. Anal. Methods 2012, 4, 895-897.

(152) Van Duuren, B. L.; Chan, T.-L.; Irani, F. M. Anal. Chem. 1968, 40 (13), 2024-2027.

(153) Nazarenko, I.; Pires, R.; Lowe, B.; Obaidy, M.; Rashtchian, A. Nucleic Acids Res. 2002, 30 (9), 2089-2195.

(154) Spiriti, J.; Binder, J. K.; Levitus, M.; Van Der Vaart, A. Biophys. J. 2011, 100 (4), 10491057.

(155) Harvey, B. J.; Levitus, M. J. Fluoresc. 2009, 19, 443-448.

(156) Jia, K.; Wan, Y.; Xia, A.; Li, S.; Gong, F.; Yang, G. J. Phys. Chem. 2007, 111, 1593-1597.

(157) Stennett, E. M. S.; Ma, N.; Van Der Vaart, A.; Levitus, M. J. Phys. Chem. B 2014, 118, $152-163$.

(158) Cowperthwaite, M. C.; Ellington, A. D. J. Mol. Evol. 2008, 67 (1), 95-102.

(159) Henry, S. H.; Bosch, F. X.; Troxell, T. C.; Bolger, P. M. Science (80-. ). 1999, 286 (5449).

(160) IARC. In Improving public health through pharmacy; Pitt, J. I., Wild, C. P., Baan, R. A., Wentzel, C. A., Gelderblom, W. C. ., Miller, J. D., Riley, R. T., Wu, F., Eds.; International 
Agency for Research on Cancer: Lyon, France, 2012; pp 53-58.

(161) Kaur, H.; Lanry Yung, L.-Y.; Lynn Addison, C. PLoS One 2012, 7 (2).

(162) Lin, P.-H.; Chen, R.-H.; Lee, C.-H.; Chang, Y.; Chen, C.-S.; Chen, W.-Y. Colloids Surfaces B Biointerfaces 2011, 88, 552-558.

(163) Gaillard, C.; Strauss, F. BMC Biochem. 2000, 1 (1), 1-7. 
$\Delta$ 